NISTIR 8147

\title{
Measuring Airborne Emissions from Cigarette Butts: Literature Review and Experimental Plan
}

Final Report to U.S. Food and Drug Administration under

Interagency Agreement \#244-15-9012

Dustin Poppendieck

Shahana Khurshid

Steven Emmerich

This publication is available free of charge from:

http://dx.doi.org/10.6028/NIST.IR.8147

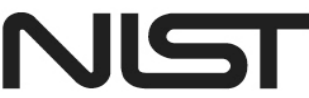

National Institute of

Standards and Technology

U.S. Department of Commerce 


\title{
Measuring Airborne Emissions from Cigarette Butts: Literature Review and Experimental Plan
} Final Report to U.S. Food and Drug Administration under Interagency Agreement \#244-15-9012

\author{
Dustin Poppendieck \\ Shahana Khurshid \\ Steven Emmerich \\ Energy and Environment Division \\ Engineering Laboratory
}

This publication is available free of charge from:

http://dx.doi.org/10.6028/NIST.IR.8147

October 2016

INCLUDES UPDATES AS OF 10-19-2016: PAGE 35

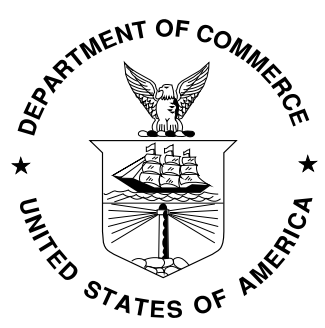

U.S. Department of Commerce Penny Pritzker, Secretary

National Institute of Standards and Technology Willie May, Under Secretary of Commerce for Standards and Technology and Director 


\section{Abstract}

Under an interagency agreement with the Food and Drug Administration (FDA), a comprehensive literature review was conducted to gather and analyze existing research related to airborne emissions from non-smoldering cigarette butts. Based on the results from the literature review, an experimental plan was developed to measure the airborne emissions from non-smoldering cigarette butts. The literature review found that: 1) Non-smoldering cigarette butts can contain many of the same chemicals found in mainstream and sidestream smoke, and they are a potential source of these chemicals in both indoor and outdoor environments; 2) A number of studies have investigated the chemicals found in cigarette butts and chemicals leached? from cigarette butts into water. However, there are very limited data on the emissions from cigarette butts into air; 3 ) The emission rates from cigarette butts into air may be minimal for some heavy chemicals (e.g., metals, tobacco-specific nitrosamines), but may be significant for more volatile chemicals (e.g., nicotine, pyridine, benzene); 4) The airborne emissions of cigarette butts may be influenced by the cigarette brand, filter material, butt length, environmental temperature, airflow around the cigarette, number of puffs during smoking, degradation of the butt, and smoking method; 5) Much more data are needed on the airborne emission rates under different conditions. Based on the information from the literature review, the proposed experimental plan aims to fill the data gaps by using a screening tool (e.g. headspace analysis) to examine the airborne emission from non-smoldering cigarette butts under various environmental conditions. Steps in the proposed investigation include development of headspace analysis methods, selection of cigarette brand, determination of butt length, generation of cigarette butts, and determination of the target compounds. The proposed experiments will be conducted under four exposure environments, including small chamber, large chamber (to mimic indoor conditions), Simulation Photo-degradation via High Energy Radiation Emission chamber (SPHERE), with ultra violet radiation to simulate accelerated aging in outdoor environments, and outdoor rooftop (to represent aging in an outdoor environment). 


\section{Contents}

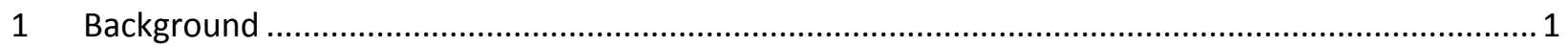

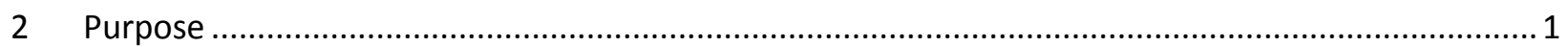

3 Literature Review on Cigarette Butt Emissions …........................................................................ 1

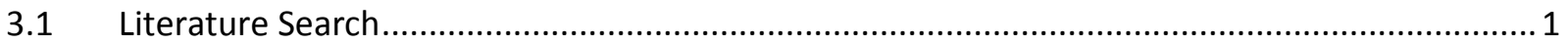

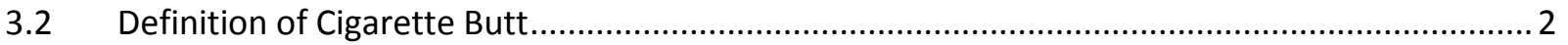

3.3 Chemical Composition of Cigarette Butts .............................................................................. 3

3.3.1 Chemicals Emitted from Cigarette Butts into Air ............................................................ 19

3.3.2 Chemicals Emitted from Cigarette Butts into Water ........................................................ 20

3.3.3 Chemicals Measured in Cigarette Butts....................................................................... 21

3.3.4 Mainstream and Sidestream Smoke Chemicals Potentially in Cigarette Butts ................. 22

3.4 Factors that Potentially Influence Airborne Emissions from Cigarette Butts ............................2 27

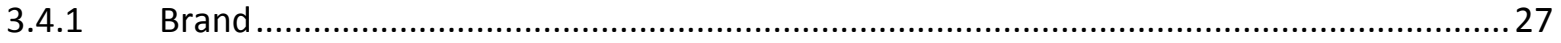

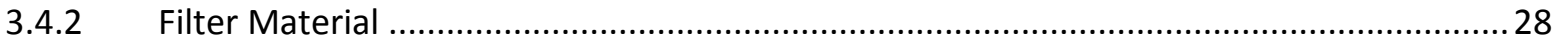

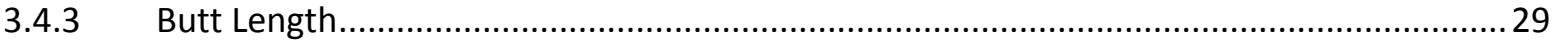

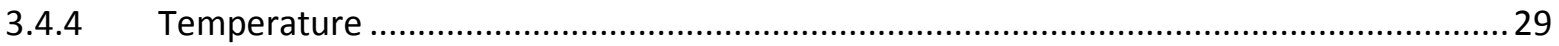

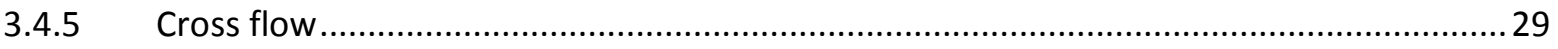

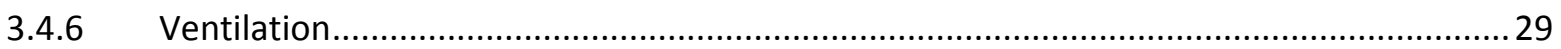

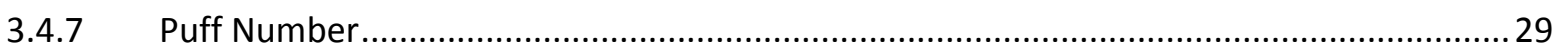

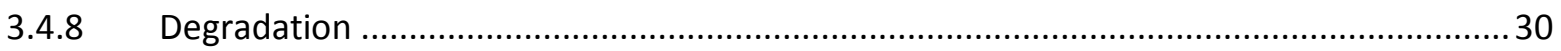

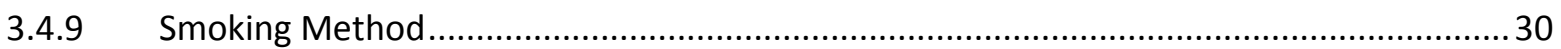

3.5 Impacts of Cigarette Butts on Human Health and Environment .............................................. 30

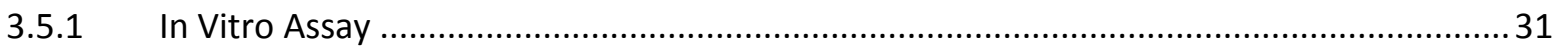

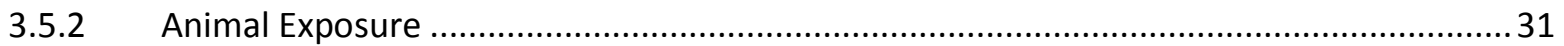

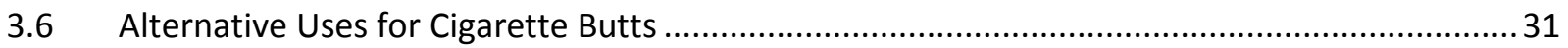

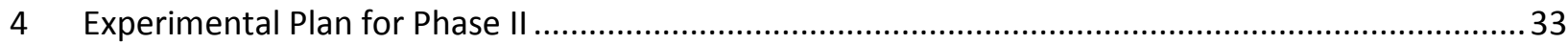

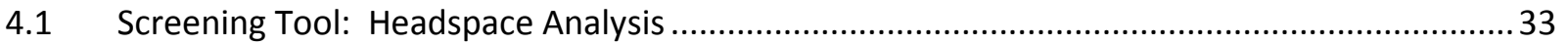

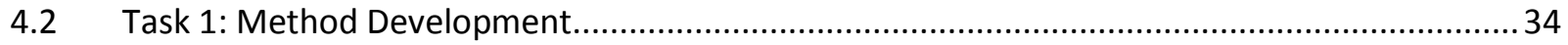

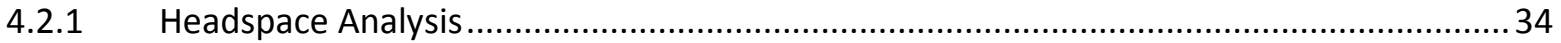

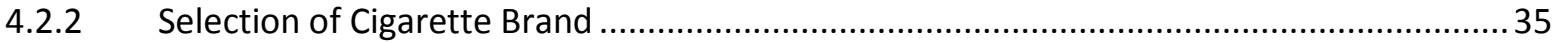

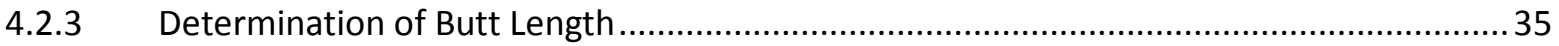

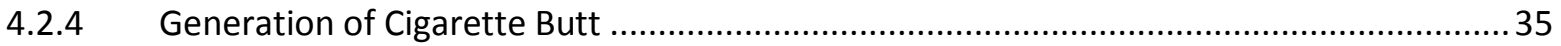

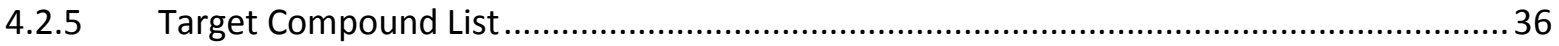




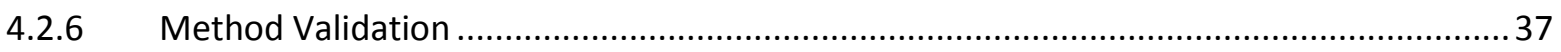

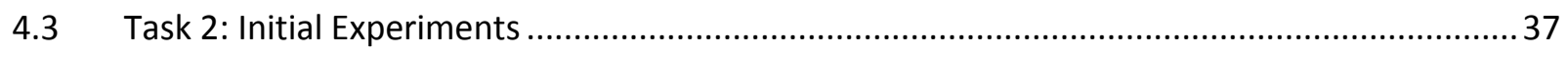

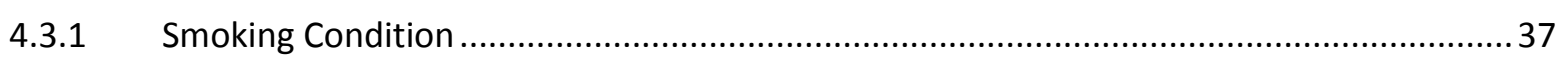

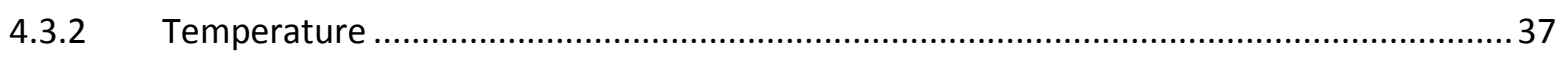

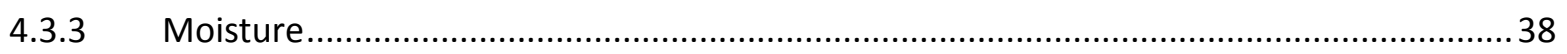

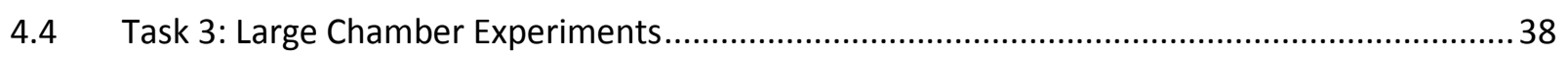

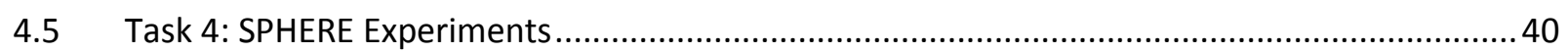

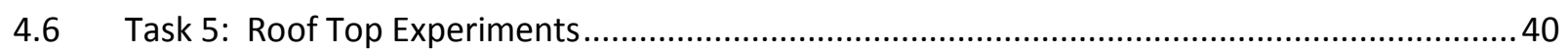

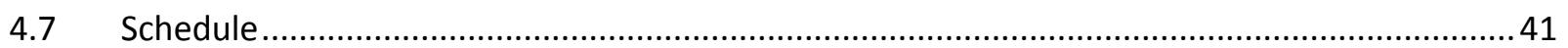

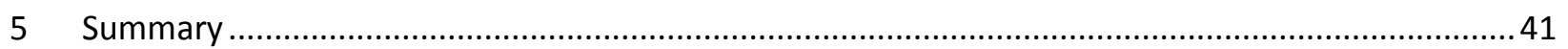

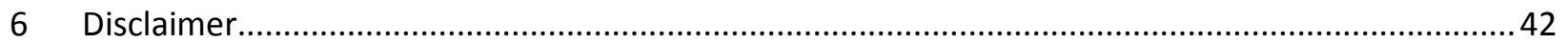

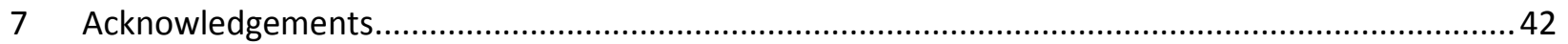

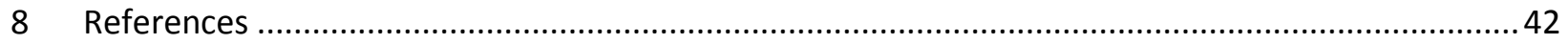




\section{Glossary}

Airborne emission: Release of pollutants into air from a solid or liquid source as it burns or volatilizes.

Automated headspace analysis: Measurement of compounds of interest from the vapor phase around a sample placed in a closed system instead of directly from the sample matrix. Automation allows for enhanced precision and sample throughput.

Chemical:

A form of matter that is composed of a particular set of elements and has characteristic properties. A chemical cannot be separated into the elements it is comprised of by physical separation methods, i.e., without breaking chemical bonds.

Cigarette butt: Defined in this document as the cigarette remaining at the conclusion of the smoldering phase following smoking. The conclusion of the smoldering phase is defined as when the entire cigarette butt reaches the ambient temperature of the environment in which it is located. Typically, the cigarette butt has three major parts: ash, unburnt tobacco, and filtration material. The cigarette butt includes any remaining paper (wrapping paper, tipping paper, and plug wrap paper).

Cigarette filter: $\quad$ Includes the filtration material, tipping paper, plug wrap paper, and adhesive. The filtration material is usually made of cellulose acetate, the cigarette filter is intended to trap less desirable components of mainstream smoke.

Cigarette tip: $\quad$ A term used to refer to cigarette filters, especially for cigarettes that people roll themselves.

Compendex Engineering Village: Database of patents and journals across disciplines. Disciplines include: electrical, civil, chemical, mechanical, mining, and general engineering in addition to applied science. This database covers engineering to a greater depth than the Web of Science database.

Compounds: $\quad$ A form of matter that is composed of two or more elements and has characteristic properties. A compound cannot be separated into the elements it is comprised of by physical separation methods, i.e., without breaking chemical bonds.

Constituents: Compounds present in a cigarette after it has been smoked.

Cross flow:

Ambient air flow surrounding the cigarette.

Ingredients:

ISI Web of Science

Compounds or additives added to cigarettes during the manufacturing process.

Databases of journals across disciplines. The disciplines include agricultural, biological, and environmental sciences, engineering, technology, applied science, medical and life sciences, and physical and chemical sciences. This database covers biological, medical, and environmental sciences to a greater depth than the Compendex Engineering Village database.

Mainstream smoke: All smoke that leaves the butt end of a cigarette during the smoking process (ISO3308 2012). 
Non-smoldering: The non-smoldering phase starts when the entire cigarette butt reaches the ambient temperature of the environment in which it is located and particles are no longer emitted from the combustion process.

Rooftop experiments: Experiments that will be conducted on the rooftop of building 226 on the NISTGaithersburg campus to measure the effect of aging in the outdoor environment on airborne emissions from cigarette butts. Several instruments are located on this rooftop to measure environmental conditions such as solar irradiance, wind speed, rain, etc.

Sidestream smoke: All smoke which leaves a cigarette during the smoking process other than from the butt end (ISO3308 2012).

SPHERE experiments: Experiments that will be conducted in the Simulation Photo-degradation via High Energy Radiation Emission chamber (SPHERE), with ultra violet radiation to simulate accelerated aging of cigarette butts in outdoor environments.

Tenax-TA tubes: Porous polymer adsorbent used for collecting airborne organic contaminants. For analysis the tubes are thermally desorbed into the inlet of a gas chromatography system.

Tobacco filter: $\quad$ A term used to refer to cigarette filters.

Ventilation: Air flow though the cigarette. 


\section{Background}

The Family Smoking Prevention and Tobacco Control Act (Tobacco Control Act) was signed into law on June 22, 2009, amending the Food, Drug and Cosmetic Act and providing the Food and Drug Administration (FDA) the authority to regulate the manufacturing, distribution, and marketing of tobacco products. More specifically, the Tobacco Control Act gives FDA the authority to, among other things, establish science and research programs to inform the development of tobacco product regulations and better understand the risks associated with tobacco use.

FDA considers the environmental impacts of its actions as an integral part of its regulatory process. The National Environmental Policy Act (NEPA) of 1969, as amended (42 U.S.C. § 4321 et seq.), directs that all agencies of the Federal Government include a detailed assessment of the environmental effects of a major Federal action significantly affecting the quality of the human environment. To comply with NEPA, according to 21 CFR 25, FDA follows guidance from the Council for Environmental Quality (CEQ) in assessing the environmental effects of any proposed action. Under 21 CFR 25.40(a), the environmental assessment shall focus on relevant issues relating to the use and disposal of FDA regulated articles. Included in the environmental considerations of tobacco product applications, FDA considers, among other factors, the environmental effects of waste generated as a result of disposal after use.

A major existing environmental consequence of the use of tobacco products, such as conventional and roll-your-own cigarettes, is the waste disposal of discarded cigarette filters or butts, which can persist in the environment for more than 10 years (Novotny and Zhao 1999). Globally, around 5 trillion cigarette butts are generated per year (Novotny and Zhao 1999, Chapman 2006, Healton et al. 2011, Bonanomi et al. 2015). Cigarette butts are some of the most common forms of litter found on beaches (Claereboudt 2004, Smith et al. 2014), near streams, night clubs (Becherucci and Pon 2014), bus stops (Wilson et al. 2014), roads and streets (Healton et al. 2011, Patel et al. 2013). Cigarette butts have been found at densities averaging more than four cigarette butts $\mathrm{m}^{-2}$ in urban environments (Seco Pon and Becherucci 2012).

The environmental toxicity of cigarette butts due to air emissions is not well studied. FDA is seeking to gather more information regarding air emissions from cigarette butts and to assess the cumulative environmental impact of filtered tobacco products.

\section{Purpose}

This report summarizes activities under an interagency agreement (\#244-15-9012) between the Food and Drug Administration (FDA) and the National Institute of Standards and Technology (NIST). The objective of that agreement was: 1 ) to conduct a literature review to gather and analyze existing research related to airborne emissions from non-smoldering cigarette butts, and 2) to develop an experimental plan to measure the airborne emissions from non-smoldering cigarette butts.

\section{Literature Review on Cigarette Butt Emissions}

A literature search, as described below, was conducted to evaluate existing literature that assessed emissions from non-smoldering cigarette butts

\subsection{Literature Search}

A literature search of papers published prior to January $20^{\text {th }}$ of 2016 was carried out using the ISI Web of Science and Compendex Engineering Village databases. As shown in Table 1, the search keywords used were "Cigarette butt*", "Cigarette filter*", "Cigarette tip*" and "Tobacco filter*". The "*" wildcard character allowed for plural words to be searched simultaneously. A total of 1793 papers were 
identified from ISI Web of Science, while 1101 papers were identified from Compendex Engineering Village. Combining the papers from the two search results and removing the duplicate papers resulted in a database with 2381 papers. Of those 2381 papers, 2186 papers were excluded since the studies performed were not related to the topics discussed in this document. Specifically, those excluded papers focused on subjects such as the act of smoking, diseases caused by the act of smoking, how to make cigarettes, experiments on rats or mice smoking, and indoor air filters to remove cigarette smoke.

Table 1. Summary of literature searches.

\begin{tabular}{|cccc|}
\hline Search database & $\begin{array}{c}\text { Search keywords } \\
\text { Web of Science }\end{array}$ & No. of papers & Search date \\
\hline & $\begin{array}{c}\text { Cigarette butt* or } \\
\text { Cigarette filter* or } \\
\text { Cigarette tip* or } \\
\text { Tobacco filter* }\end{array}$ & 1793 & $01-20-16$ \\
\hline $\begin{array}{c}\text { Compendex } \\
\text { Engineering Village }\end{array}$ & $\begin{array}{l}\text { Cigarette butt* or } \\
\text { Cigarette filter* or } \\
\text { Cigarette tip* or } \\
\text { Tobacco filter* }\end{array}$ & 1101 & $01-20-16$ \\
\hline
\end{tabular}

None of the remaining reviewed 195 articles directly studied airborne emission from non-smoldering cigarette butts in indoor or outdoor environmental conditions. Four articles reported the emission from cigarette butts into the headspace of sample vials at elevated temperatures. The detailed summary of these four papers is described in section 3.3.1. As a result of the limited data set on airborne emission, the focus of the literature review was widened to investigate all possible emissions from cigarette butts to provide context to the experimental plan. Consequently, the 195 papers were scrutinized for this report. Of those 195 papers, 121 relevant articles which discussed the definition of cigarette butts, chemical composition of cigarette butts, factors that potentially influence cigarette butt airborne emissions, impacts of cigarette butts on human health and environment, and alternative uses for cigarette butts, have been cited in this document.

\subsection{Definition of Cigarette Butt}

A definition of a cigarette butt is needed to accurately discuss emissions from cigarette butts. ISO 3308 (2012) (Routine analytical cigarette-smoking machine - Definitions and standard conditions) defines a butt length as the "length of unburnt cigarette remaining at the moment when smoking is stopped." For the purpose of this document, this definition of a cigarette butt will be the cigarette remaining at the conclusion of the smoldering phase following smoking (i.e. the remaining cigarette butt has reached the temperature of the environment it is in). For the remainder of this document the term cigarette butt will refer to a non-smoldering cigarette butt.

A cigarette butt has three major components: 1) ash, 2) unburned tobacco and 3) filtration material (Figure 1). These three major components are wrapped by three types of paper (wrapping paper, tipping paper, and plug wrap paper). 


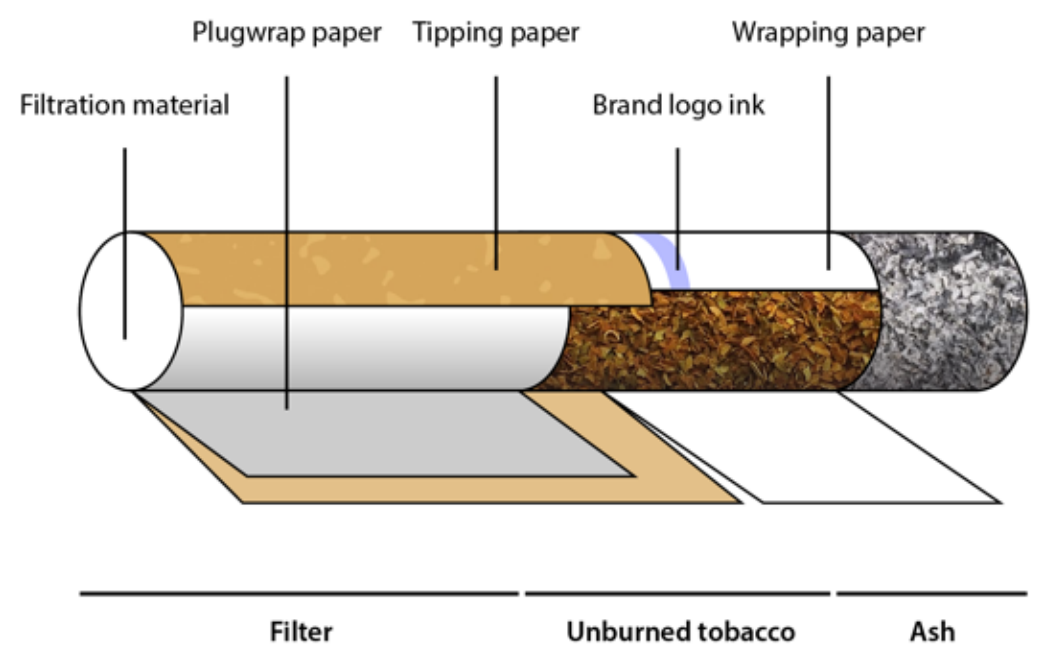

Figure 1: Components of cigarette butt.

\subsection{Chemical Composition of Cigarette Butts}

Since there are limited data on airborne emissions from cigarette butts, it is important to characterize the chemical composition of cigarette butts in order to assess their potential airborne emissions. The chemicals in cigarette butts can be the original chemicals in the unsmoked cigarettes or the pyrolysis and distillation products deposited in the cigarette butts. Each component of a cigarette butt (e.g., ash, unburned tobacco, filter and paper) can contain different chemicals that could possibly emit as the cigarette butt ages.

The chemical composition of the ash and the unburned tobacco depends on the types of tobacco, how the cigarette was smoked, and the mass transfer behavior of combustion products along the cigarette. The diameter of the cigarette (Coggins et al. 2013) and the permeability of the wrapping paper will influence the burned condition (Xia et al. 2012). Depending on the burning conditions, the combustion temperature in a cigarette can reach up to $800^{\circ} \mathrm{C}$ (Hertz et al. 2012). Different chemicals are produced at different temperatures and times during the burning process. Hydrocarbons (e.g., benzene and naphthalene) are formed by pyrolysis above $600{ }^{\circ} \mathrm{C}$, while phenols are generated at $400{ }^{\circ} \mathrm{C}$. In contrast, nicotine is released by distillation at temperatures below $300^{\circ} \mathrm{C}$ (Baker 1987).

Chemicals found in cigarette filters will depend on the filter design. Filters are designed to capture various less desirable components of mainstream smoke, with each cigarette brand using different strategies. The type of filter material will influence the initial chemicals present in the cigarette butt that can potentially be emitted. Most filters are made of cellulose acetate. However, researchers have examined the performance of filters consisting of activated carbon, tert-butylhydroquinone (TBHQ), vitamin C, carotene, grape seed extract, Ginkgo biloba extract, dried hemoglobin, and polyurethane foam/aluminum hydroxide hybrid (Smith et al. 1997, Valavanidis and Haralambous 2001, Masoudi Soltani and Kazemi Yazdi 2012, Liu et al. 2013, Petraru et al. 2013, Salman et al. 2014, Soltani et al. 2015).

It should be noted that some additives may be added to the unburned tobacco to improve their sensory properties and increase attractiveness to smokers. These additives include single compounds such as 
glycerol, propylene glycol, menthol, vanillin, diammonium hydrogenphosphate, and n-propyl-phydroxybenzoate, as well as complex additive mixtures such as cocoa, licorice, and mint oil (Merckel and Pragst 2007, Huang et al. 2014). Some of the additives may remain in the cigarette butt. Similar to other original chemicals, the additive transfer behavior to the cigarette butt is dependent on the molecular weight and boiling point of the additive, the configuration of the cigarette, and other factors such as the chemical polarity of the additive (Huang et al. 2014).

Regardless of the different composition of different cigarette butts, even for cigarette butts from the same pack, different chemicals can be detected when the butt is exposed to different environments or measured using different methods. The following sections (3.3.1 to 3.3.4) summarize the studies that have reported chemicals related to cigarette butts or related to individual components of cigarette butts (e.g. filter, unburned tobacco, ash and paper), including: 1) emissions from cigarette butts or individual butt components into air, 2) emissions from cigarette butts or individual butt components into water, 3 ) extraction of cigarette butts and direct analysis of cigarette butts or individual butt components, and 4) components in mainstream or sidestream smoke that potentially exist in cigarette butts. Table 2 summarizes the chemicals reported to be directly associated with cigarette butts and the analytical methods used in the first three groups of studies. The fourth group of studies are excluded from Table 2 , since the experiments did not positively associate the measured chemicals with cigarette butts. 
Table 2. Summary of detected chemicals reported in cigarette butt emission and extraction studies. Acronyms are defined in Table 3. Chemicals highlighted in bold and marked using asterisk are on the United States Food and Drug Administrations' Harmful and Potentially

Harmful Constituents in Tobacco Products and Tobacco Smoke: Established List

\begin{tabular}{|c|c|c|c|c|c|c|c|}
\hline Class & Chemical & CAS & $\begin{array}{l}\mathrm{MW}^{\mathrm{a}} \\
(\mathrm{g} / \mathrm{mol})\end{array}$ & Component & Mediab $^{b}$ & Summary of method & Reference \\
\hline Alcohols & Ethanol & $64-17-5$ & 46.1 & $\begin{array}{l}\text { Tipping } \\
\text { paper }\end{array}$ & Air & $\begin{array}{l}\text { Incubate a cigarette butt in a headspace vial, analyze } \\
\text { the air in the headspace by GC-MS }\end{array}$ & (Huang et al. 2014) \\
\hline Alcohols & 2-Furfuryl Alcohol & $98-00-0$ & 98.1 & $\begin{array}{l}\text { Cigarette } \\
\text { butt }\end{array}$ & Air & $\begin{array}{l}\text { Incubate a cigarette butt in a headspace vial, collect } \\
\text { the sample with Tenax GC, analyze the sample by GC- } \\
\text { FID }\end{array}$ & (Fukuhara et al. 1985) \\
\hline Alcohols & Isopropanol & $67-63-0$ & 60.1 & $\begin{array}{l}\text { Tipping } \\
\text { paper }\end{array}$ & Air & $\begin{array}{l}\text { Incubate a cigarette butt in a headspace vial, analyze } \\
\text { the air in the headspace by GC-MS }\end{array}$ & (Huang et al. 2014) \\
\hline Alcohols & Methanol & $67-56-1$ & 32.0 & $\begin{array}{l}\text { Cigarette } \\
\text { Filter }\end{array}$ & Air & $\begin{array}{l}\text { Incubate a cigarette butt in a headspace vial, analyze } \\
\text { the air in the headspace by GC-FID }\end{array}$ & (You et al. 2014) \\
\hline Alcohols & 1-methoxy-2-propanol & $107-98-2$ & 90.1 & $\begin{array}{l}\text { Tipping } \\
\text { paper }\end{array}$ & Air & $\begin{array}{l}\text { Incubate a cigarette butt in a headspace vial, analyze } \\
\text { the air in the headspace by GC-MS }\end{array}$ & (Huang et al. 2014) \\
\hline Alcohols & 1-ethoxy-2-propanol & $1569-02-4$ & 104.2 & $\begin{array}{l}\text { Tipping } \\
\text { paper }\end{array}$ & Air & $\begin{array}{l}\text { Incubate a cigarette butt in a headspace vial, analyze } \\
\text { the air in the headspace by GC-MS }\end{array}$ & (Huang et al. 2014) \\
\hline Alkaloids & $\begin{array}{l}\text { 7-Carbaldehyde } \\
\text { camptothecin }\end{array}$ & $80758-83-4$ & 376.4 & $\begin{array}{l}\text { Cigarette } \\
\text { butt }\end{array}$ & Water & $\begin{array}{l}\text { Leach into distilled water, analyze the leachate by LC- } \\
\text { MS }\end{array}$ & (Zhao et al. 2010a) \\
\hline $\begin{array}{l}\text { Aromatic } \\
\text { amines }\end{array}$ & Nicotine* & $22083-74-5$ & 162.2 & $\begin{array}{l}\text { Cigarette } \\
\text { butt }\end{array}$ & Water & $\begin{array}{l}\text { Leach into distilled water, analyze the leachate by LC- } \\
\text { MS }\end{array}$ & $\begin{array}{l}\text { (Zhao et al. 2010a), } \\
\text { (Zhao et al. 2010b) }\end{array}$ \\
\hline $\begin{array}{l}\text { Aromatic } \\
\text { amines }\end{array}$ & Nicotine* & $22083-74-5$ & 162.2 & $\begin{array}{l}\text { Cigarette } \\
\text { butt }\end{array}$ & Water & $\begin{array}{l}\text { Leach into purified water and simulated rainwater, } \\
\text { analyze the leachate by LC-MS-MS }\end{array}$ & (Green et al. 2014) \\
\hline $\begin{array}{l}\text { Aromatic } \\
\text { amines }\end{array}$ & Hexaconazole & 79983-71-4 & 314.2 & $\begin{array}{l}\text { Cigarette } \\
\text { butt }\end{array}$ & Water & $\begin{array}{l}\text { Leach into distilled water, analyze the leachate by LC- } \\
\text { MS }\end{array}$ & (Zhao et al. 2010a) \\
\hline $\begin{array}{l}\text { Aromatic } \\
\text { amines }\end{array}$ & Imidocarb & $27885-92-3$ & 348.4 & $\begin{array}{l}\text { Cigarette } \\
\text { butt }\end{array}$ & Water & $\begin{array}{l}\text { Leach into distilled water, analyze the leachate by LC- } \\
\text { MS }\end{array}$ & (Zhao et al. 2010a) \\
\hline $\begin{array}{l}\text { Aromatic } \\
\text { amines }\end{array}$ & Cotinine & $486-56-6$ & 176.2 & $\begin{array}{l}\text { Cigarette } \\
\text { butt }\end{array}$ & Water & $\begin{array}{l}\text { Leach into distilled water, analyze the leachate by LC- } \\
\text { MS }\end{array}$ & (Zhao et al. 2010b) \\
\hline
\end{tabular}




\begin{tabular}{|c|c|c|c|c|c|c|c|}
\hline Class & Chemical & CAS & $\begin{array}{l}\mathrm{MW}^{\mathrm{a}} \\
(\mathrm{g} / \mathrm{mol})\end{array}$ & Component & Media $^{b}$ & Summary of method & Reference \\
\hline $\begin{array}{l}\text { Aromatic } \\
\text { amines }\end{array}$ & $\begin{array}{l}\text { 2-(Pyridin-3-yl) } \\
\text { Pyrrolidine-1- } \\
\text { Carbaldehyde }\end{array}$ & $3000-81-5$ & 176.2 & $\begin{array}{l}\text { Cigarette } \\
\text { butt }\end{array}$ & Water & $\begin{array}{l}\text { Leach into distilled water, analyze the leachate by LC- } \\
\text { MS }\end{array}$ & (Zhao et al. 2010a) \\
\hline $\begin{array}{l}\text { Aromatic } \\
\text { amines }\end{array}$ & Sulfadoxine & $2447-57-6$ & 310.3 & $\begin{array}{l}\text { Cigarette } \\
\text { butt }\end{array}$ & Water & $\begin{array}{l}\text { Leach into distilled water, analyze the leachate by LC- } \\
\text { MS }\end{array}$ & (Zhao et al. 2010a) \\
\hline $\begin{array}{l}\text { Aromatic } \\
\text { amines }\end{array}$ & $\begin{array}{l}\text { 5-(4,6-Dichloropyridin-3- } \\
\text { yl)-Pyridine-1(2H)- } \\
\text { Carboxamide }^{\mathrm{a}}\end{array}$ & & 270 & $\begin{array}{l}\text { Cigarette } \\
\text { butt }\end{array}$ & Water & $\begin{array}{l}\text { Leach into distilled water, analyze the leachate by LC- } \\
\text { MS }\end{array}$ & (Zhao et al. 2010b) \\
\hline Carbonyls & Acetol & $116-09-6$ & 74.1 & $\begin{array}{l}\text { Cigarette } \\
\text { butt }\end{array}$ & Air & $\begin{array}{l}\text { Incubate a cigarette butt in a headspace vial, collect } \\
\text { the sample with Tenax GC, analyze the sample by GC- } \\
\text { FID }\end{array}$ & (Fukuhara et al. 1985) \\
\hline Carbonyls & Acetone* & $67-64-1$ & 58.1 & $\begin{array}{l}\text { Tipping } \\
\text { paper }\end{array}$ & Air & $\begin{array}{l}\text { Incubate a cigarette butt in a headspace vial, analyze } \\
\text { the air in the headspace by GC-MS }\end{array}$ & (Huang et al. 2014) \\
\hline Carbonyls & Acetone* & $67-64-1$ & 58.1 & $\begin{array}{l}\text { Cigarette } \\
\text { filter }\end{array}$ & $\begin{array}{l}\text { Extract } \\
\text { solution }\end{array}$ & $\begin{array}{l}\text { Extract with acetonitrile aqueous solution, analyze the } \\
\text { extract by LC-MS-MS }\end{array}$ & (Yu et al. 2013) \\
\hline Carbonyls & Butyl acetate & $123-86-4$ & 116.2 & $\begin{array}{l}\text { Tipping } \\
\text { paper }\end{array}$ & Air & $\begin{array}{l}\text { Incubate a cigarette butt in a headspace vial, analyze } \\
\text { the air in the headspace by GC-MS }\end{array}$ & (Huang et al. 2014) \\
\hline Carbonyls & Cyclohexanone & $108-94-1$ & 98.1 & $\begin{array}{l}\text { Tipping } \\
\text { paper }\end{array}$ & Air & $\begin{array}{l}\text { Incubate a cigarette butt in a headspace vial, analyze } \\
\text { the air in the headspace by GC-MS }\end{array}$ & (Huang et al. 2014) \\
\hline Carbonyls & 2-Pentanone & $107-87-9$ & 86.1 & $\begin{array}{l}\text { Cigarette } \\
\text { butt }\end{array}$ & Air & $\begin{array}{l}\text { Incubate a cigarette butt in a headspace vial, collect } \\
\text { the sample with Tenax GC, analyze the sample by GC- } \\
\text { FID }\end{array}$ & (Fukuhara et al. 1985) \\
\hline Carbonyls & n-propanol & $71-23-8$ & 60.1 & $\begin{array}{l}\text { Tipping } \\
\text { paper }\end{array}$ & Air & $\begin{array}{l}\text { Incubate a cigarette butt in a headspace vial, analyze } \\
\text { the air in the headspace by GC-MS }\end{array}$ & (Huang et al. 2014) \\
\hline Carbonyls & n-propyl acetate & $109-60-4$ & 102.1 & $\begin{array}{l}\text { Tipping } \\
\text { paper }\end{array}$ & Air & $\begin{array}{l}\text { Incubate a cigarette butt in a headspace vial, analyze } \\
\text { the air in the headspace by GC-MS }\end{array}$ & (Huang et al. 2014) \\
\hline Carbonyls & Cyclopentanone & $120-92-3$ & 84.1 & $\begin{array}{l}\text { Cigarette } \\
\text { butt }\end{array}$ & Air & $\begin{array}{l}\text { Incubate a cigarette butt in a headspace vial, collect } \\
\text { the sample with Tenax GC, analyze the sample by GC- } \\
\text { FID }\end{array}$ & (Fukuhara et al. 1985) \\
\hline
\end{tabular}




\begin{tabular}{|c|c|c|c|c|c|c|c|}
\hline Class & Chemical & CAS & $\begin{array}{l}\mathrm{MW}^{\mathrm{a}} \\
(\mathrm{g} / \mathrm{mol})\end{array}$ & Component & Media $^{b}$ & Summary of method & Reference \\
\hline Carbonyls & 2-Cyclopentenone & $930-30-3$ & 82.1 & $\begin{array}{l}\text { Cigarette } \\
\text { butt }\end{array}$ & Air & $\begin{array}{l}\text { Incubate a cigarette butt in a headspace vial, collect } \\
\text { the sample with Tenax GC, analyze the sample by GC- } \\
\text { FID }\end{array}$ & (Fukuhara et al. 1985) \\
\hline Carbonyls & 2-Methylcyclo pentenone & $1120-73-6$ & 96.1 & $\begin{array}{l}\text { Cigarette } \\
\text { butt }\end{array}$ & Air & $\begin{array}{l}\text { Incubate a cigarette butt in a headspace vial, collect } \\
\text { the sample with Tenax GC, analyze the sample by GC- } \\
\text { FID }\end{array}$ & (Fukuhara et al. 1985) \\
\hline Carbonyls & 4-methyl-2-pentanone & $108-10-1$ & 100.2 & $\begin{array}{l}\text { Tipping } \\
\text { paper }\end{array}$ & Air & $\begin{array}{l}\text { Incubate a cigarette butt in a headspace vial, analyze } \\
\text { the air in the headspace by GC-MS }\end{array}$ & (Huang et al. 2014) \\
\hline Carbonyls & Methyl n-Butyl Ketone & $591-78-6$ & 100.2 & $\begin{array}{l}\text { Tipping } \\
\text { paper }\end{array}$ & Air & $\begin{array}{l}\text { Incubate a cigarette butt in a headspace vial, analyze } \\
\text { the air in the headspace by GC-MS }\end{array}$ & (Huang et al. 2014) \\
\hline Carbonyls & n-butyl alcohol & $71-36-3$ & 74.1 & $\begin{array}{l}\text { Tipping } \\
\text { paper }\end{array}$ & Air & $\begin{array}{l}\text { Incubate a cigarette butt in a headspace vial, analyze } \\
\text { the air in the headspace by GC-MS }\end{array}$ & (Huang et al. 2014) \\
\hline Carbonyls & Ethyl acetate & $141-78-6$ & 88.1 & $\begin{array}{l}\text { Tipping } \\
\text { paper }\end{array}$ & Air & $\begin{array}{l}\text { Incubate a cigarette butt in a headspace vial, analyze } \\
\text { the air in the headspace by GC-MS }\end{array}$ & (Huang et al. 2014) \\
\hline Carbonyls & 3-Furaldehyde & $498-60-2$ & 96.1 & $\begin{array}{l}\text { Cigarette } \\
\text { butt }\end{array}$ & Air & $\begin{array}{l}\text { Incubate a cigarette butt in a headspace vial, collect } \\
\text { the sample with Tenax GC, analyze the sample by GC- } \\
\text { FID }\end{array}$ & (Fukuhara et al. 1985) \\
\hline Carbonyls & 2-Furaldehyde & 98-01-1 & 96.1 & $\begin{array}{l}\text { Cigarette } \\
\text { butt }\end{array}$ & Air & $\begin{array}{l}\text { Incubate a cigarette butt in a headspace vial, collect } \\
\text { the sample with Tenax GC, analyze the sample by GC- } \\
\text { FID }\end{array}$ & (Fukuhara et al. 1985) \\
\hline Carbonyls & Formaldehyde* & $50-00-0$ & 30 & $\begin{array}{l}\text { Cigarette } \\
\text { filter }\end{array}$ & $\begin{array}{l}\text { Extract } \\
\text { solution }\end{array}$ & $\begin{array}{l}\text { Extract with acetonitrile aqueous solution, analyze the } \\
\text { extract by LC-MS-MS }\end{array}$ & (Yu et al. 2013) \\
\hline Carbonyls & Isopropyl acetate & $108-21-4$ & 102.1 & $\begin{array}{l}\text { Tipping } \\
\text { paper }\end{array}$ & Air & $\begin{array}{l}\text { Incubate a cigarette butt in a headspace vial, analyze } \\
\text { the air in the headspace by GC-MS }\end{array}$ & (Huang et al. 2014) \\
\hline Carbonyls & Acetaldehyde* & $75-07-0$ & 44.1 & $\begin{array}{l}\text { Cigarette } \\
\text { filter }\end{array}$ & $\begin{array}{l}\text { Extract } \\
\text { solution }\end{array}$ & $\begin{array}{l}\text { Extract with acetonitrile aqueous solution, analyze the } \\
\text { extract by LC-MS-MS }\end{array}$ & (Yu et al. 2013) \\
\hline Carbonyls & Acrolein* & $107-02-8$ & 56.1 & $\begin{array}{l}\text { Cigarette } \\
\text { filter }\end{array}$ & $\begin{array}{l}\text { Extract } \\
\text { solution }\end{array}$ & $\begin{array}{l}\text { Extract with acetonitrile aqueous solution, analyze the } \\
\text { extract by LC-MS-MS }\end{array}$ & (Yu et al. 2013) \\
\hline
\end{tabular}




\begin{tabular}{|c|c|c|c|c|c|c|c|}
\hline Class & Chemical & CAS & $\begin{array}{l}\mathrm{MW}^{\mathrm{a}} \\
(\mathrm{g} / \mathrm{mol})\end{array}$ & Component & Media $^{b}$ & Summary of method & Reference \\
\hline Carbonyls & Propionaldehyde* & $123-38-6$ & 58.1 & $\begin{array}{l}\text { Cigarette } \\
\text { filter }\end{array}$ & $\begin{array}{l}\text { Extract } \\
\text { solution }\end{array}$ & $\begin{array}{l}\text { Extract with acetonitrile aqueous solution, analyze the } \\
\text { extract by LC-MS-MS }\end{array}$ & (Yu et al. 2013) \\
\hline Carbonyls & Protoanemonin & $108-28-1$ & 96.08 & $\begin{array}{l}\text { Cigarette } \\
\text { butt }\end{array}$ & Air & $\begin{array}{l}\text { Incubate a cigarette butt in a headspace vial, collect } \\
\text { the sample with Tenax GC, analyze the sample by GC- } \\
\text { FID }\end{array}$ & (Fukuhara et al. 1985) \\
\hline Carbonyls & Crotonaldehyde* & $4170-30-3$ & 70.09 & $\begin{array}{l}\text { Cigarette } \\
\text { filter }\end{array}$ & $\begin{array}{l}\text { Extract } \\
\text { solution }\end{array}$ & $\begin{array}{l}\text { Extract with acetonitrile aqueous solution, analyze the } \\
\text { extract by LC-MS-MS }\end{array}$ & (Yu et al. 2013) \\
\hline Carbonyls & 2-Butanone & $78-93-3$ & 72.1 & $\begin{array}{l}\text { Cigarette } \\
\text { filter }\end{array}$ & $\begin{array}{l}\text { Extract } \\
\text { solution }\end{array}$ & $\begin{array}{l}\text { Extract with acetonitrile aqueous solution, analyze the } \\
\text { extract by LC-MS-MS }\end{array}$ & (Yu et al. 2013) \\
\hline Carbonyls & Butyraldehyde & $123-72-8$ & 72.1 & $\begin{array}{l}\text { Cigarette } \\
\text { filter }\end{array}$ & $\begin{array}{l}\text { Extract } \\
\text { solution }\end{array}$ & $\begin{array}{l}\text { Extract with acetonitrile aqueous solution, analyze the } \\
\text { extract by LC-MS-MS }\end{array}$ & (Yu et al. 2013) \\
\hline Carbonyls & Acetol Formate & $116-09-6$ & 74.1 & $\begin{array}{l}\text { Cigarette } \\
\text { butt }\end{array}$ & Air & $\begin{array}{l}\text { Incubate a cigarette butt in a headspace vial, collect } \\
\text { the sample with Tenax GC, analyze the sample by GC- } \\
\text { FID }\end{array}$ & (Fukuhara et al. 1985) \\
\hline Carbonyls & $\begin{array}{l}\text { 3-Methyl-2- } \\
\text { Cyclopentenone }\end{array}$ & $2758-18-1$ & 96.1 & $\begin{array}{l}\text { Cigarette } \\
\text { butt }\end{array}$ & Air & $\begin{array}{l}\text { Incubate a cigarette butt in a headspace vial, collect } \\
\text { the sample with Tenax GC, analyze the sample by GC- } \\
\text { FID }\end{array}$ & (Fukuhara et al. 1985) \\
\hline Carbonyls & 2,3-Pentanedione & $600-14-6$ & 100.1 & $\begin{array}{l}\text { Cigarette } \\
\text { butt }\end{array}$ & Air & $\begin{array}{l}\text { Incubate a cigarette butt in a headspace vial, collect } \\
\text { the sample with Tenax GC, analyze the sample by GC- } \\
\text { FID }\end{array}$ & (Fukuhara et al. 1985) \\
\hline Carbonyls & $\begin{array}{l}\text { 5-(4-Hydroxy Pyridin-3- } \\
\text { yl)-Pyridine-1(2H)- } \\
\text { Carboxamide }\end{array}$ & & 217 & $\begin{array}{l}\text { Cigarette } \\
\text { butt }\end{array}$ & Water & $\begin{array}{l}\text { Leach into distilled water, analyze the leachate by LC- } \\
\text { MS }\end{array}$ & (Zhao et al. 2010a) \\
\hline Carbonyls & 7-Keto-Benzo(a)pyrene & & 272 & $\begin{array}{l}\text { Cigarette } \\
\text { butt }\end{array}$ & Water & $\begin{array}{l}\text { Leach into distilled water, analyze the leachate by LC- } \\
\text { MS }\end{array}$ & (Zhao et al. 2010a) \\
\hline Hydrocarbons & Ethyl Benzene* & $100-41-4$ & 106.2 & $\begin{array}{l}\text { Cigarette } \\
\text { butt }\end{array}$ & Air & $\begin{array}{l}\text { Incubate a cigarette butt in a headspace vial, collect } \\
\text { the sample with Tenax GC, analyze the sample by GC- } \\
\text { FID }\end{array}$ & (Fukuhara et al. 1985) \\
\hline Hydrocarbons & Ethyl benzene* & $100-41-4$ & 106.2 & Tipping & Air & Incubate a cigarette butt in a headspace vial, analyze & (Huang et al. 2014) \\
\hline
\end{tabular}




\begin{tabular}{|c|c|c|c|c|c|c|c|}
\hline Class & Chemical & CAS & $\begin{array}{l}\mathrm{MW}^{\mathrm{a}} \\
(\mathrm{g} / \mathrm{mol})\end{array}$ & Component & Mediab & Summary of method & Reference \\
\hline & & & & paper & & the air in the headspace by GC-MS & \\
\hline Hydrocarbons & Ethyl Benzene* & $100-41-4$ & 106.2 & $\begin{array}{l}\text { Cigarette } \\
\text { butt }\end{array}$ & Air & $\begin{array}{l}\text { Incubate a cigarette butt in a headspace vial, analyze } \\
\text { the air in the headspace by GC-MS }\end{array}$ & (Ji et al. 2015) \\
\hline Hydrocarbons & Benzene* & $71-43-2$ & 78.1 & $\begin{array}{l}\text { Tipping } \\
\text { paper }\end{array}$ & Air & $\begin{array}{l}\text { Incubate a cigarette butt in a headspace vial, analyze } \\
\text { the air in the headspace by GC-MS }\end{array}$ & (Huang et al. 2014) \\
\hline Hydrocarbons & Benzene* & $71-43-2$ & 78.1 & $\begin{array}{l}\text { Cigarette } \\
\text { butt }\end{array}$ & Air & $\begin{array}{l}\text { Incubate a cigarette butt in a headspace vial, analyze } \\
\text { the air in the headspace by GC-MS }\end{array}$ & (Ji et al. 2015) \\
\hline Hydrocarbons & 3,3-Dimethyl-1-Butene & $558-37-2$ & 84.2 & $\begin{array}{l}\text { Cigarette } \\
\text { butt }\end{array}$ & Air & $\begin{array}{l}\text { Incubate a cigarette butt in a headspace vial, collect } \\
\text { the sample with Tenax GC, analyze the sample by GC- } \\
\text { FID }\end{array}$ & (Fukuhara et al. 1985) \\
\hline Hydrocarbons & Pyridine & $110-86-1$ & 79.1 & $\begin{array}{l}\text { Cigarette } \\
\text { butt }\end{array}$ & Air & $\begin{array}{l}\text { Incubate a cigarette butt in a headspace vial, collect } \\
\text { the sample with Tenax GC, analyze the sample by GC- } \\
\text { FID }\end{array}$ & (Fukuhara et al. 1985) \\
\hline Hydrocarbons & Cyanobenzene & $100-47-0$ & 103.1 & $\begin{array}{l}\text { Cigarette } \\
\text { butt }\end{array}$ & Air & $\begin{array}{l}\text { Incubate a cigarette butt in a headspace vial, collect } \\
\text { the sample with Tenax GC, analyze the sample by GC- } \\
\text { FID }\end{array}$ & (Fukuhara et al. 1985) \\
\hline Hydrocarbons & Cyclooctatetraene & $629-20-9$ & 104.2 & $\begin{array}{l}\text { Cigarette } \\
\text { butt }\end{array}$ & Air & $\begin{array}{l}\text { Incubate a cigarette butt in a headspace vial, collect } \\
\text { the sample with Tenax GC, analyze the sample by GC- } \\
\text { FID }\end{array}$ & (Fukuhara et al. 1985) \\
\hline Hydrocarbons & Isocapronitrile & $542-54-1$ & 97.2 & $\begin{array}{l}\text { Cigarette } \\
\text { butt }\end{array}$ & Air & $\begin{array}{l}\text { Incubate a cigarette butt in a headspace vial, collect } \\
\text { the sample with Tenax GC, analyze the sample by GC- } \\
\text { FID }\end{array}$ & (Fukuhara et al. 1985) \\
\hline Hydrocarbons & Toluene* & $108-88-3$ & 92.1 & $\begin{array}{l}\text { Cigarette } \\
\text { butt }\end{array}$ & Air & $\begin{array}{l}\text { Incubate a cigarette butt in a headspace vial, analyze } \\
\text { the air in the headspace by GC-MS }\end{array}$ & (Ji et al. 2015) \\
\hline Hydrocarbons & Toluene* & $108-88-3$ & 92.1 & $\begin{array}{l}\text { Tipping } \\
\text { paper }\end{array}$ & Air & $\begin{array}{l}\text { Incubate a cigarette butt in a headspace vial, analyze } \\
\text { the air in the headspace by GC-MS }\end{array}$ & (Huang et al. 2014) \\
\hline Hydrocarbons & Toluene* & $108-88-6$ & 92.1 & $\begin{array}{l}\text { Cigarette } \\
\text { butt }\end{array}$ & Air & $\begin{array}{l}\text { Incubate a cigarette butt in a headspace vial, collect } \\
\text { the sample with Tenax GC, analyze the sample by GC- } \\
\text { FID }\end{array}$ & (Fukuhara et al. 1985) \\
\hline
\end{tabular}




\begin{tabular}{|c|c|c|c|c|c|c|c|}
\hline Class & Chemical & CAS & $\begin{array}{l}\mathrm{MW}^{\mathrm{a}} \\
(\mathrm{g} / \mathrm{mol})\end{array}$ & Component & Media $^{b}$ & Summary of method & Reference \\
\hline Hydrocarbons & p-Xylene & $106-42-3$ & 106.2 & $\begin{array}{l}\text { Cigarette } \\
\text { butt }\end{array}$ & Air & $\begin{array}{l}\text { Incubate a cigarette butt in a headspace vial, analyze } \\
\text { the air in the headspace by GC-MS }\end{array}$ & (Ji et al. 2015) \\
\hline Hydrocarbons & m-Xylene & $108-38-3$ & 106.2 & $\begin{array}{l}\text { Cigarette } \\
\text { butt }\end{array}$ & Air & $\begin{array}{l}\text { Incubate a cigarette butt in a headspace vial, analyze } \\
\text { the air in the headspace by GC-MS }\end{array}$ & (Ji et al. 2015) \\
\hline Hydrocarbons & m-Xylene & $108-38-3$ & 106.2 & $\begin{array}{l}\text { Tipping } \\
\text { paper }\end{array}$ & Air & $\begin{array}{l}\text { Incubate a cigarette butt in a headspace vial, analyze } \\
\text { the air in the headspace by GC-MS }\end{array}$ & (Huang et al. 2014) \\
\hline Hydrocarbons & o-Xylene & $95-47-6$ & 106.2 & $\begin{array}{l}\text { Tipping } \\
\text { paper }\end{array}$ & Air & $\begin{array}{l}\text { Incubate a cigarette butt in a headspace vial, analyze } \\
\text { the air in the headspace by GC-MS }\end{array}$ & (Huang et al. 2014) \\
\hline Hydrocarbons & o-Xylene & $95-47-6$ & 106.2 & $\begin{array}{l}\text { Cigarette } \\
\text { butt }\end{array}$ & Air & $\begin{array}{l}\text { Incubate a cigarette butt in a headspace vial, analyze } \\
\text { the air in the headspace by GC-MS }\end{array}$ & (Ji et al. 2015) \\
\hline Hydrocarbons & Styrene* & $100-42-5$ & 104.2 & $\begin{array}{l}\text { Tipping } \\
\text { paper }\end{array}$ & Air & $\begin{array}{l}\text { Incubate a cigarette butt in a headspace vial, analyze } \\
\text { the air in the headspace by GC-MS }\end{array}$ & (Huang et al. 2014) \\
\hline Hydrocarbons & Styrene* & $100-42-5$ & 104.2 & $\begin{array}{l}\text { Cigarette } \\
\text { butt }\end{array}$ & Air & $\begin{array}{l}\text { Incubate a cigarette butt in a headspace vial, analyze } \\
\text { the air in the headspace by GC-MS }\end{array}$ & (Ji et al. 2015) \\
\hline Hydrocarbons & Xylene & $1330-20-7$ & 106.2 & $\begin{array}{l}\text { Cigarette } \\
\text { butt }\end{array}$ & Air & $\begin{array}{l}\text { Incubate a cigarette butt in a headspace vial, collect } \\
\text { the sample with Tenax GC, analyze the sample by GC- } \\
\text { FID }\end{array}$ & (Fukuhara et al. 1985) \\
\hline Insecticides & Maleic Hydrazide & $123-33-1$ & 112.1 & $\begin{array}{l}\text { Cigarette } \\
\text { butt }\end{array}$ & $\begin{array}{l}\text { Extract } \\
\text { solution }\end{array}$ & $\begin{array}{l}\text { Extract with methanol, dissolve the extract in DMF } \\
\text { and silylate with BSTFA, analyze the silylated extract } \\
\text { by GC-MS }\end{array}$ & (Zhang et al. 2012) \\
\hline Insecticides & Chlorantraniliprole & $500008-45-7$ & 483.2 & $\begin{array}{l}\text { Cigarette } \\
\text { butt }\end{array}$ & $\begin{array}{l}\text { Extract } \\
\text { solution }\end{array}$ & Extract with acetonitrile, radioassay the extract by LSC & $\begin{array}{l}\text { (Gaddamidi et al. } \\
\text { 2011) }\end{array}$ \\
\hline Insecticides & Imidacloprid & $138261-41-3$ & 255.7 & $\begin{array}{l}\text { Cigarette } \\
\text { butt }\end{array}$ & $\begin{array}{l}\text { Extract } \\
\text { solution }\end{array}$ & Extract with acetone, radioassay the extract by LSC & (Clark et al. 1998) \\
\hline Metals & Aluminum (Al) & $7429-90-5$ & 27 & $\begin{array}{l}\text { Cigarette } \\
\text { butt }\end{array}$ & Water & $\begin{array}{l}\text { Leach into aqueous solution, analyze the leachate by } \\
\text { ICP-OES }\end{array}$ & $\begin{array}{l}\text { (Moerman and Potts } \\
\text { 2011) }\end{array}$ \\
\hline Metals & Antimony (Sb) & $7440-36-0$ & 121.8 & $\begin{array}{l}\text { Cigarette } \\
\text { butt }\end{array}$ & $\begin{array}{l}\text { Cigarette } \\
\text { butt }\end{array}$ & $\begin{array}{l}\text { Analyze different components of cigarette butt } \\
\text { (unburned tobacco, ash, filter, cigarette paper) }\end{array}$ & (Wu et al. 1997) \\
\hline
\end{tabular}




\begin{tabular}{|c|c|c|c|c|c|c|c|}
\hline Class & Chemical & CAS & $\begin{array}{l}\mathrm{MW}^{\mathrm{a}} \\
(\mathrm{g} / \mathrm{mol})\end{array}$ & Component & Media $^{\mathbf{b}}$ & Summary of method & Reference \\
\hline & & & & & & directly by INNA & \\
\hline Metals & Arsenic (As)* & $7440-38-2$ & 74.9 & $\begin{array}{l}\text { Cigarette } \\
\text { butt }\end{array}$ & $\begin{array}{l}\text { Cigarette } \\
\text { butt }\end{array}$ & $\begin{array}{l}\text { Analyze different components of cigarette butt } \\
\text { (unburned tobacco, ash, filter, cigarette paper) } \\
\text { directly by INNA }\end{array}$ & (Wu et al. 1997) \\
\hline Metals & Arsenic (As)* & $7440-38-2$ & 74.9 & $\begin{array}{l}\text { Cigarette } \\
\text { filter }\end{array}$ & $\begin{array}{l}\text { Extract } \\
\text { solution }\end{array}$ & $\begin{array}{l}\text { Extract with } \mathrm{HNO}_{3} \text { and } \mathrm{H}_{2} \mathrm{O}_{2} \text { by heating in water bath, } \\
\text { analyze the extract by } \mathrm{HG} \text {-AFS }\end{array}$ & (Wang et al. 2007) \\
\hline Metals & Barium $(\mathrm{Ba})^{*}$ & $7440-39-3$ & 137.3 & $\begin{array}{l}\text { Cigarette } \\
\text { butt }\end{array}$ & Water & $\begin{array}{l}\text { Leach into aqueous solution, analyze the leachate by } \\
\text { ICP-OES }\end{array}$ & $\begin{array}{l}\text { (Moerman and Potts } \\
\text { 2011) }\end{array}$ \\
\hline Metals & Cadmium $(\mathrm{Cd})^{*}$ & $7440-43-9$ & 112.4 & $\begin{array}{l}\text { Cigarette } \\
\text { butt }\end{array}$ & Water & $\begin{array}{l}\text { Leach into aqueous solution, analyze the leachate by } \\
\text { ICP-OES }\end{array}$ & $\begin{array}{l}\text { (Moerman and Potts } \\
\text { 2011) }\end{array}$ \\
\hline Metals & Cadmium $(\mathrm{Cd})^{*}$ & $7440-43-9$ & 112.4 & $\begin{array}{l}\text { Cigarette } \\
\text { butt }\end{array}$ & $\begin{array}{l}\text { Extract } \\
\text { solution }\end{array}$ & $\begin{array}{l}\text { Extract with } \mathrm{HNO}_{3}-\mathrm{HClO}_{4} \text { by wet ashing technique, } \\
\text { analyze the extract by FAAS }\end{array}$ & (Pelit et al. 2013) \\
\hline Metals & Cadmium (Cd)* & $7440-43-9$ & 112.4 & $\begin{array}{l}\text { Cigarette } \\
\text { butt }\end{array}$ & $\begin{array}{l}\text { Cigarette } \\
\text { butt }\end{array}$ & $\begin{array}{l}\text { Analyze different components of cigarette butt } \\
\text { (unburned tobacco, ash, filter, cigarette paper) } \\
\text { directly by INNA }\end{array}$ & (Wu et al. 1997) \\
\hline Metals & Cadmium (Cd)* & $7440-43-9$ & 112.4 & $\begin{array}{l}\text { Cigarette } \\
\text { butt }\end{array}$ & $\begin{array}{l}\text { Cigarette } \\
\text { butt }\end{array}$ & $\begin{array}{l}\text { Analyze different components of cigarette butt } \\
\text { (unburned tobacco, ash, filter, cigarette paper) } \\
\text { directly by INNA }\end{array}$ & (Wu et al. 1997) \\
\hline Metals & Cadmium $(\mathrm{Cd})^{*}$ & $7440-43-9$ & 112.4 & $\begin{array}{l}\text { Cigarette } \\
\text { butt }\end{array}$ & $\begin{array}{l}\text { Extract } \\
\text { solution }\end{array}$ & $\begin{array}{l}\text { Extract with } 69 \% \mathrm{HNO}_{3} \text { and } 36 \% \mathrm{HCl} \text { using a } \\
\text { microwave system, analyze the extract by FAAS }\end{array}$ & $\begin{array}{l}\text { (Galazyn-Sidorczuk et } \\
\text { al. 2008) }\end{array}$ \\
\hline Metals & Cadmium $(\mathrm{Cd})^{*}$ & $7440-43-9$ & 112.4 & $\begin{array}{l}\text { Unburned } \\
\text { tobacco }\end{array}$ & $\begin{array}{l}\text { Extract } \\
\text { solution }\end{array}$ & $\begin{array}{l}\text { Extract with } \mathrm{HCl} \text {, analyze the extract by solid surface } \\
\text { fluorescence using nylon membranes coated with } \\
\text { carbon nanotubes }\end{array}$ & $\begin{array}{l}\text { (Carolina Talio et al. } \\
\text { 2013) }\end{array}$ \\
\hline Metals & Chromium (Cr)* & $7440-47-3$ & 52 & $\begin{array}{l}\text { Cigarette } \\
\text { butt }\end{array}$ & $\begin{array}{l}\text { Extract } \\
\text { solution }\end{array}$ & Extract with $\mathrm{HNO}_{3}$, analyze the extract by ICP-AES & $\begin{array}{l}\text { (Wang and Finlayson- } \\
\text { Pitts 2003) }\end{array}$ \\
\hline Metals & Cobalt (Co)* & $7440-48-4$ & 58.9 & $\begin{array}{l}\text { Cigarette } \\
\text { butt }\end{array}$ & $\begin{array}{l}\text { Cigarette } \\
\text { butt }\end{array}$ & $\begin{array}{l}\text { Analyze different components of cigarette butt } \\
\text { (unburned tobacco, ash, filter, cigarette paper) } \\
\text { directly by INNA }\end{array}$ & (Wu et al. 1997) \\
\hline
\end{tabular}




\begin{tabular}{|c|c|c|c|c|c|c|c|}
\hline Class & Chemical & CAS & $\begin{array}{l}\mathrm{MW}^{\mathrm{a}} \\
(\mathrm{g} / \mathrm{mol})\end{array}$ & Component & Media $^{b}$ & Summary of method & Reference \\
\hline Metals & Copper (Cu) & $7440-50-8$ & 63.6 & $\begin{array}{l}\text { Cigarette } \\
\text { butt }\end{array}$ & $\begin{array}{l}\text { Extract } \\
\text { solution }\end{array}$ & $\begin{array}{l}\text { Extract with } \mathrm{HNO}_{3}-\mathrm{HClO}_{4} \text { by wet ashing technique, } \\
\text { analyze the extract by FAAS }\end{array}$ & (Pelit et al. 2013) \\
\hline Metals & Mercury (Hg)* & $7439-97-6$ & 200.6 & $\begin{array}{l}\text { Cigarette } \\
\text { filter }\end{array}$ & $\begin{array}{l}\text { Extract } \\
\text { solution }\end{array}$ & $\begin{array}{l}\text { Extract with } \mathrm{HNO}_{3} \text { and } \mathrm{H}_{2} \mathrm{O}_{2} \text { by heating in water bath, } \\
\text { analyze the extract by } \mathrm{HG}-\mathrm{AFS}\end{array}$ & (Wang et al. 2007) \\
\hline Metals & Mercury (Hg)* & $7439-97-6$ & 200.6 & $\begin{array}{l}\text { Cigarette } \\
\text { butt }\end{array}$ & $\begin{array}{l}\text { Cigarette } \\
\text { butt }\end{array}$ & $\begin{array}{l}\text { Analyze the cigarette butt directly by mercury } \\
\text { analyzer AMA- } 254\end{array}$ & $\begin{array}{l}\text { (Kowalski and } \\
\text { Wiercinski 2009) }\end{array}$ \\
\hline Metals & Iron (Fe) & $7439-89-6$ & 55.9 & $\begin{array}{l}\text { Cigarette } \\
\text { butt }\end{array}$ & $\begin{array}{l}\text { Extract } \\
\text { solution }\end{array}$ & Extract with $\mathrm{HNO}$, analyze the extract by ICP-AES & $\begin{array}{l}\text { (Wang and Finlayson- } \\
\text { Pitts 2003) }\end{array}$ \\
\hline Metals & Lead $(\mathrm{Pb})^{*}$ & $7439-92-1$ & 207.2 & $\begin{array}{l}\text { Cigarette } \\
\text { butt }\end{array}$ & $\begin{array}{l}\text { Extract } \\
\text { solution }\end{array}$ & $\begin{array}{l}\text { Extract with } 69 \% \mathrm{HNO} \text { and } 36 \% \mathrm{HCl} \text { using a } \\
\text { microwave system, analyze the extract by FAAS }\end{array}$ & $\begin{array}{l}\text { (Galazyn-Sidorczuk et } \\
\text { al. 2008) }\end{array}$ \\
\hline Metals & Lead $(\mathrm{Pb})^{*}$ & $7439-92-1$ & 207.2 & $\begin{array}{l}\text { Cigarette } \\
\text { filter }\end{array}$ & $\begin{array}{l}\text { Extract } \\
\text { solution }\end{array}$ & $\begin{array}{l}\text { Extract with solvent using a microwave system, } \\
\text { analyze the extract by FAAS }\end{array}$ & (Huang et al. 2013) \\
\hline Metals & Lead $(\mathrm{Pb})^{*}$ & $7439-92-1$ & 207.2 & $\begin{array}{l}\text { Cigarette } \\
\text { paper }\end{array}$ & $\begin{array}{l}\text { Extract } \\
\text { solution }\end{array}$ & $\begin{array}{l}\text { Ash the cigarette paper with microwave ashing at } 450 \\
{ }^{\circ} \mathrm{C} \text {, extract the ash with nitric acid, analyze the extract } \\
\text { by FAAS }\end{array}$ & (Wu et al. 2012) \\
\hline Metals & Lead $(\mathrm{Pb})^{*}$ & $7439-92-1$ & 207.2 & $\begin{array}{l}\text { Cigarette } \\
\text { butt }\end{array}$ & Water & $\begin{array}{l}\text { Leach into aqueous solution, analyze the leachate by } \\
\text { ICP-OES }\end{array}$ & $\begin{array}{l}\text { (Moerman and Potts } \\
\text { 2011) }\end{array}$ \\
\hline Metals & Manganese (Mn) & $7439-96-5$ & 54.9 & $\begin{array}{l}\text { Cigarette } \\
\text { butt }\end{array}$ & Water & $\begin{array}{l}\text { Leach into aqueous solution, analyze the leachate by } \\
\text { ICP-OES }\end{array}$ & $\begin{array}{l}\text { (Moerman and Potts } \\
\text { 2011) }\end{array}$ \\
\hline Metals & Manganese $(\mathrm{Mn})$ & $7439-96-5$ & 54.9 & $\begin{array}{l}\text { Cigarette } \\
\text { butt }\end{array}$ & $\begin{array}{l}\text { Extract } \\
\text { solution }\end{array}$ & $\begin{array}{l}\text { Extract with } \mathrm{HNO}_{3}-\mathrm{HClO}_{4} \text { by wet ashing technique, } \\
\text { analyze the extract by FAAS }\end{array}$ & (Pelit et al. 2013) \\
\hline Metals & Nickel (Ni)* & $7440-02-0$ & 58.7 & $\begin{array}{l}\text { Cigarette } \\
\text { butt }\end{array}$ & Water & $\begin{array}{l}\text { Leach into aqueous solution, analyze the leachate by } \\
\text { ICP-OES }\end{array}$ & $\begin{array}{l}\text { (Moerman and Potts } \\
\text { 2011) }\end{array}$ \\
\hline Metals & Potassium (K) & $7440-09-7$ & 39.1 & $\begin{array}{l}\text { Cigarette } \\
\text { butt }\end{array}$ & $\begin{array}{l}\text { Cigarette } \\
\text { butt }\end{array}$ & $\begin{array}{l}\text { Analyze different components of cigarette butt } \\
\text { (unburned tobacco, ash, filter, cigarette paper) } \\
\text { directly by INNA }\end{array}$ & (Wu et al. 1997) \\
\hline Metals & Strontium (Sr) & $7440-24-6$ & 87.6 & $\begin{array}{l}\text { Cigarette } \\
\text { butt }\end{array}$ & Water & $\begin{array}{l}\text { Leach into aqueous solution, analyze the leachate by } \\
\text { ICP-OES }\end{array}$ & $\begin{array}{l}\text { (Moerman and Potts } \\
\text { 2011) }\end{array}$ \\
\hline
\end{tabular}




\begin{tabular}{|c|c|c|c|c|c|c|c|}
\hline Class & Chemical & CAS & $\begin{array}{l}\mathrm{MW}^{\mathrm{a}} \\
(\mathrm{g} / \mathrm{mol})\end{array}$ & Component & Media $^{b}$ & Summary of method & Reference \\
\hline Metals & Titanium (Ti) & $7440-32-6$ & 47.9 & $\begin{array}{l}\text { Cigarette } \\
\text { butt }\end{array}$ & Water & $\begin{array}{l}\text { Leach into aqueous solution, analyze the leachate by } \\
\text { ICP-OES }\end{array}$ & $\begin{array}{l}\text { (Moerman and Potts } \\
\text { 2011) }\end{array}$ \\
\hline Metals & Zinc $(Z n)$ & $7440-66-6$ & 65.4 & $\begin{array}{l}\text { Cigarette } \\
\text { butt }\end{array}$ & $\begin{array}{l}\text { Extract } \\
\text { solution }\end{array}$ & Extract with $\mathrm{HNO}_{3}$, analyze the extract by ICP-AES & $\begin{array}{l}\text { (Wang and Finlayson- } \\
\text { Pitts 2003) }\end{array}$ \\
\hline Metals & Zinc $(Z n)$ & $7440-66-6$ & 65.4 & $\begin{array}{l}\text { Cigarette } \\
\text { butt }\end{array}$ & $\begin{array}{l}\text { Extract } \\
\text { solution }\end{array}$ & $\begin{array}{l}\text { Extract with } \mathrm{HNO}-\mathrm{HClO} 4 \text { by wet ashing technique, } \\
\text { analyze the extract by FAAS }\end{array}$ & (Pelit et al. 2013) \\
\hline Metals & Zinc $(Z n)$ & $7440-66-6$ & 65.4 & $\begin{array}{l}\text { Cigarette } \\
\text { butt }\end{array}$ & $\begin{array}{l}\text { Cigarette } \\
\text { butt }\end{array}$ & $\begin{array}{l}\text { Analyze different components of cigarette butt } \\
\text { (unburned tobacco, ash, filter, cigarette paper) } \\
\text { directly by INNA }\end{array}$ & (Wu et al. 1997) \\
\hline Nitrosamines & $\begin{array}{l}\text { N-Nitrosonornicotine } \\
\text { (NNN)* }\end{array}$ & $16543-55-8$ & 177 & $\begin{array}{l}\text { Cigarette } \\
\text { butt }\end{array}$ & Water & $\begin{array}{l}\text { Leach into distilled water, analyze the leachate by LC- } \\
\text { MS }\end{array}$ & $\begin{array}{l}\text { (Zhao et al. 2010a, } \\
\text { Zhao et al. 2010b) }\end{array}$ \\
\hline Nitrosamines & TSNAs $^{c}$ & & & $\begin{array}{l}\text { Cigarette } \\
\text { filter }\end{array}$ & $\begin{array}{l}\text { Extract } \\
\text { solution }\end{array}$ & $\begin{array}{l}\text { Extracting the filter with mixture of deuterated } \\
\text { water/dimethyl sulfoxide, analyze the extract by NMR }\end{array}$ & (Verdolotti et al. 2012) \\
\hline NPAHs & 1,3-dinitronaphthalene & & & $\begin{array}{l}\text { Cigarette } \\
\text { filter }\end{array}$ & $\begin{array}{l}\text { Extract } \\
\text { solution }\end{array}$ & Extract with toluene, analyze the extract by EM-MS & (John Dane et al. 2002) \\
\hline NPAHS & 9-nitroanthracene & & & $\begin{array}{l}\text { Cigarette } \\
\text { filter }\end{array}$ & $\begin{array}{l}\text { Extract } \\
\text { solution }\end{array}$ & Extract with toluene, analyze the extract by EM-MS & (John Dane et al. 2002) \\
\hline NPAHs & Nitrobenzene* & & & $\begin{array}{l}\text { Cigarette } \\
\text { filter }\end{array}$ & $\begin{array}{l}\text { Extract } \\
\text { solution }\end{array}$ & Extract with toluene, analyze the extract by EM-MS & (John Dane et al. 2002) \\
\hline PAHs & Anthracene & $120-12-7$ & 178.2 & $\begin{array}{l}\text { Cigarette } \\
\text { filter }\end{array}$ & $\begin{array}{l}\text { Extract } \\
\text { solution }\end{array}$ & $\begin{array}{l}\text { Extract with acetonitrile and water, analyze the } \\
\text { extract using SPME and HPLC }\end{array}$ & (Demirci 2014) \\
\hline PAHs & Benzo(a)pyrene* & $50-32-8$ & 252.3 & $\begin{array}{l}\text { Cigarette } \\
\text { butt }\end{array}$ & Water & $\begin{array}{l}\text { Leach into distilled water, analyze the leachate by LC- } \\
\text { MS }\end{array}$ & (Zhao et al. 2010a) \\
\hline PAHs & Benzo[a]pyrene* & $50-32-8$ & 252.3 & $\begin{array}{l}\text { Cigarette } \\
\text { filter }\end{array}$ & $\begin{array}{l}\text { Extract } \\
\text { solution }\end{array}$ & $\begin{array}{l}\text { Strip the wrapping paper, extract with acetone, clean } \\
\text { up by solid phase extraction, analyze the extract by } \\
\text { HPLC-FLD }\end{array}$ & (Ding et al. 2014) \\
\hline PAHs & Benzo[a]pyrene* & $50-32-8$ & 252.3 & $\begin{array}{l}\text { Cigarette } \\
\text { filter }\end{array}$ & $\begin{array}{l}\text { Extract } \\
\text { solution }\end{array}$ & $\begin{array}{l}\text { Extract with acetonitrile, analyze the extract by LC- } \\
\text { MS-MS }\end{array}$ & (Zhang et al. 2014) \\
\hline
\end{tabular}




\begin{tabular}{|c|c|c|c|c|c|c|c|}
\hline Class & Chemical & CAS & $\begin{array}{l}\mathrm{MW}^{\mathrm{a}} \\
(\mathrm{g} / \mathrm{mol})\end{array}$ & Component & Media $^{b}$ & Summary of method & Reference \\
\hline PAHs & Benzo[a]pyrene* & $50-32-8$ & 252.3 & $\begin{array}{l}\text { Cigarette } \\
\text { filter }\end{array}$ & $\begin{array}{l}\text { Extract } \\
\text { solution }\end{array}$ & $\begin{array}{l}\text { Extract with acetonitrile and water, analyze the } \\
\text { extract using SPME and HPLC }\end{array}$ & (Demirci 2014) \\
\hline PAHs & Benz[a]anthracene* & $56-55-3$ & 228.3 & $\begin{array}{l}\text { Cigarette } \\
\text { filter }\end{array}$ & $\begin{array}{l}\text { Extract } \\
\text { solution }\end{array}$ & $\begin{array}{l}\text { Extract with acetonitrile and water, analyze the } \\
\text { extract using SPME and HPLC }\end{array}$ & (Demirci 2014) \\
\hline PAHs & Benzo[b]fluoranthene* & $205-99-2$ & 252.3 & $\begin{array}{l}\text { Cigarette } \\
\text { filter }\end{array}$ & $\begin{array}{l}\text { Extract } \\
\text { solution }\end{array}$ & $\begin{array}{l}\text { Extract with acetonitrile and water, analyze the } \\
\text { extract using SPME and HPLC }\end{array}$ & (Demirci 2014) \\
\hline PAHs & Fluoranthene & $206-44-0$ & 202.3 & $\begin{array}{l}\text { Cigarette } \\
\text { filter }\end{array}$ & $\begin{array}{l}\text { Extract } \\
\text { solution }\end{array}$ & $\begin{array}{l}\text { Extract with acetonitrile and water, analyze the } \\
\text { extract using SPME and HPLC }\end{array}$ & (Demirci 2014) \\
\hline PAHs & Pyrene & $129-00-0$ & 202.3 & $\begin{array}{l}\text { Cigarette } \\
\text { filter }\end{array}$ & $\begin{array}{l}\text { Extract } \\
\text { solution }\end{array}$ & $\begin{array}{l}\text { Extract with acetonitrile and water, analyze the } \\
\text { extract using SPME and HPLC }\end{array}$ & (Demirci 2014) \\
\hline Phenols & Catechol* & $120-80-9$ & 110.1 & $\begin{array}{l}\text { Cigarette } \\
\text { filter }\end{array}$ & $\begin{array}{l}\text { Extract } \\
\text { solution }\end{array}$ & $\begin{array}{l}\text { Extract with mix solution of acetic acid, methanol } \\
\text { water, analyze the extract by HPLC }\end{array}$ & (Hu et al. 2015) \\
\hline Phenols & Hydroquinone & $123-31-9$ & 110.1 & $\begin{array}{l}\text { Cigarette } \\
\text { filter }\end{array}$ & $\begin{array}{l}\text { Extract } \\
\text { solution }\end{array}$ & $\begin{array}{l}\text { Extract with mix solution of acetic acid, methanol } \\
\text { water, analyze the extract by HPLC }\end{array}$ & (Hu et al. 2015) \\
\hline Phenols & m-cresol* & $108-39-4$ & 108.1 & $\begin{array}{l}\text { Cigarette } \\
\text { filter }\end{array}$ & $\begin{array}{l}\text { Extract } \\
\text { solution }\end{array}$ & $\begin{array}{l}\text { Extract with mix solution of acetic acid, methanol } \\
\text { water, analyze the extract by HPLC }\end{array}$ & (Hu et al. 2015) \\
\hline Phenols & o-cresol* & $95-48-7$ & 108.1 & $\begin{array}{l}\text { Cigarette } \\
\text { filter }\end{array}$ & $\begin{array}{l}\text { Extract } \\
\text { solution }\end{array}$ & $\begin{array}{l}\text { Extract with mix solution of acetic acid, methanol } \\
\text { water, analyze the extract by HPLC }\end{array}$ & (Hu et al. 2015) \\
\hline Phenols & p-cresol* & $106-44-5$ & 108.1 & $\begin{array}{l}\text { Cigarette } \\
\text { filter }\end{array}$ & $\begin{array}{l}\text { Extract } \\
\text { solution }\end{array}$ & $\begin{array}{l}\text { Extract with mix solution of acetic acid, methanol } \\
\text { water, analyze the extract by HPLC }\end{array}$ & (Hu et al. 2015) \\
\hline Phenols & Phenol* & $108-39-4$ & 108.1 & $\begin{array}{l}\text { Cigarette } \\
\text { filter }\end{array}$ & $\begin{array}{l}\text { Extract } \\
\text { solution }\end{array}$ & $\begin{array}{l}\text { Extract with mix solution of acetic acid, methanol } \\
\text { water, analyze the extract by HPLC }\end{array}$ & (Hu et al. 2015) \\
\hline Phenols & Resorcinol & $108-95-2$ & 94.1 & $\begin{array}{l}\text { Cigarette } \\
\text { filter }\end{array}$ & $\begin{array}{l}\text { Extract } \\
\text { solution }\end{array}$ & $\begin{array}{l}\text { Extract with mix solution of acetic acid, methanol } \\
\text { water, analyze the extract by HPLC }\end{array}$ & (Hu et al. 2015) \\
\hline Phenols & Rutin & $153-18-4$ & 610.5 & $\begin{array}{l}\text { Cigarette } \\
\text { butt }\end{array}$ & Water & $\begin{array}{l}\text { Leach into distilled water, analyze the leachate by LC- } \\
\text { MS }\end{array}$ & $\begin{array}{l}\text { (Zhao et al. 2010a, } \\
\text { Zhao et al. 2010b) }\end{array}$ \\
\hline Phenols & Rutin & $153-18-4$ & 610.5 & $\begin{array}{l}\text { Cigarette } \\
\text { filter }\end{array}$ & $\begin{array}{l}\text { Extract } \\
\text { solution }\end{array}$ & $\begin{array}{l}\text { Extract with methanol, analyze the extract with HPLC- } \\
\text { UV }\end{array}$ & (Sun et al. 2012) \\
\hline
\end{tabular}




\begin{tabular}{|c|c|c|c|c|c|c|c|}
\hline Class & Chemical & CAS & $\begin{array}{l}\mathrm{MW}^{\mathrm{a}} \\
(\mathrm{g} / \mathrm{mol})\end{array}$ & Component & Media $^{b}$ & Summary of method & Reference \\
\hline Phenols & $\begin{array}{l}\text { 2,2-Dimethyl-2,3- } \\
\text { Dihydrobenzofuran-7-ol }\end{array}$ & $1563-38-8$ & 164.2 & $\begin{array}{l}\text { Cigarette } \\
\text { butt }\end{array}$ & Water & $\begin{array}{l}\text { Leach into distilled water, analyze the leachate by LC- } \\
\text { MS }\end{array}$ & (Zhao et al. 2010b) \\
\hline Phenols & $\begin{array}{l}\text { 1,5-Dihydroxy- } \\
\text { Anthraquinon }\end{array}$ & $117-12-4$ & 240.2 & $\begin{array}{l}\text { Cigarette } \\
\text { butt }\end{array}$ & Water & $\begin{array}{l}\text { Leach into distilled water, analyze the leachate by LC- } \\
\text { MS }\end{array}$ & (Zhao et al. 2010b) \\
\hline Phthalates & Dibutyl Phthalate & $84-74-2$ & 278.3 & $\begin{array}{l}\text { Cigarette } \\
\text { filter }\end{array}$ & $\begin{array}{l}\text { Extract } \\
\text { solution }\end{array}$ & $\begin{array}{l}\text { Ultrasonic extract with water and ethanol mixture, } \\
\text { analyze the extract by GC-MS }\end{array}$ & (Zhang et al. 2012) \\
\hline Phthalates & Diisobutyl Phthalate & $84-69-5$ & 278.3 & $\begin{array}{l}\text { Cigarette } \\
\text { filter }\end{array}$ & $\begin{array}{l}\text { Extract } \\
\text { solution }\end{array}$ & $\begin{array}{l}\text { Ultrasonic extract with water and ethanol mixture, } \\
\text { analyze the extract by GC-MS }\end{array}$ & (Zhang et al. 2012) \\
\hline Phthalates & $\begin{array}{l}\text { Di(2-ethylhexyl) } \\
\text { phthalate }\end{array}$ & $117-81-7$ & 390.6 & $\begin{array}{l}\text { Cigarette } \\
\text { filter }\end{array}$ & $\begin{array}{l}\text { Extract } \\
\text { solution }\end{array}$ & $\begin{array}{l}\text { Ultrasonic extract with water and ethanol mixture, } \\
\text { analyze the extract by GC-MS }\end{array}$ & (Zhang et al. 2012) \\
\hline Pyrazines & Pyrazine & $290-37-9$ & 80.1 & $\begin{array}{l}\text { Cigarette } \\
\text { butt }\end{array}$ & Air & $\begin{array}{l}\text { Incubate a cigarette butt in a headspace vial, collect } \\
\text { the sample with Tenax GC, analyze the sample by GC- } \\
\text { FID }\end{array}$ & (Fukuhara et al. 1985) \\
\hline Pyrazines & 2-Methylpyrazine & $109-08-0$ & 94.1 & $\begin{array}{l}\text { Cigarette } \\
\text { butt }\end{array}$ & Air & $\begin{array}{l}\text { Incubate a cigarette butt in a headspace vial, collect } \\
\text { the sample with Tenax GC, analyze the sample by GC- } \\
\text { FID }\end{array}$ & (Fukuhara et al. 1985) \\
\hline Pyrroles & N-Methylpyrrole & $96-54-8$ & 81.1 & $\begin{array}{l}\text { Cigarette } \\
\text { butt }\end{array}$ & Air & $\begin{array}{l}\text { Incubate a cigarette butt in a headspace vial, collect } \\
\text { the sample with Tenax GC, analyze the sample by GC- } \\
\text { FID }\end{array}$ & (Fukuhara et al. 1985) \\
\hline Pyrroles & Pyrrole & $109-97-7$ & 67.1 & $\begin{array}{l}\text { Cigarette } \\
\text { butt }\end{array}$ & Air & $\begin{array}{l}\text { Incubate a cigarette butt in a headspace vial, collect } \\
\text { the sample with Tenax GC, analyze the sample by GC- } \\
\text { FID }\end{array}$ & (Fukuhara et al. 1985) \\
\hline Pyrroles & 3-Methylpyrrole & $616-43-3$ & 81.1 & $\begin{array}{l}\text { Cigarette } \\
\text { butt }\end{array}$ & Air & $\begin{array}{l}\text { Incubate a cigarette butt in a headspace vial, collect } \\
\text { the sample with Tenax GC, analyze the sample by GC- } \\
\text { FID }\end{array}$ & (Fukuhara et al. 1985) \\
\hline Pyrroles & 2-Methylpyrrole & $636-41-9$ & 81.1 & $\begin{array}{l}\text { Cigarette } \\
\text { butt }\end{array}$ & Air & $\begin{array}{l}\text { Incubate a cigarette butt in a headspace vial, collect } \\
\text { the sample with Tenax GC, analyze the sample by GC- } \\
\text { FID }\end{array}$ & (Fukuhara et al. 1985) \\
\hline Terpenes & Beta-Carotene-4,4'-Dione & $472-61-7$ & 596.8 & Cigarette & Water & Leach into distilled water, analyze the leachate by LC- & (Zhao et al. 2010a, \\
\hline
\end{tabular}




\begin{tabular}{|c|c|c|c|c|c|c|c|}
\hline Class & Chemical & CAS & $\begin{array}{l}\mathrm{MW}^{\mathrm{a}} \\
(\mathrm{g} / \mathrm{mol})\end{array}$ & Component & Media $^{b}$ & Summary of method & Reference \\
\hline & & & & butt & & MS & Zhao et al. 2010b) \\
\hline Terpenes & Xanthophylls & $127-40-2$ & 568.9 & $\begin{array}{l}\text { Cigarette } \\
\text { butt }\end{array}$ & Water & $\begin{array}{l}\text { Leach into distilled water, analyze the leachate by LC- } \\
\text { MS }\end{array}$ & (Zhao et al. 2010a) \\
\hline Terpenes & Limonene & $5989-27-5$ & 136.2 & $\begin{array}{l}\text { Cigarette } \\
\text { butt }\end{array}$ & Air & $\begin{array}{l}\text { Incubate a cigarette butt in a headspace vial, collect } \\
\text { the sample with Tenax GC, analyze the sample by GC- } \\
\text { FID }\end{array}$ & (Fukuhara et al. 1985) \\
\hline Terpenoids & Solanesol & $13190-97-1$ & 631.1 & $\begin{array}{l}\text { Cigarette } \\
\text { filter }\end{array}$ & $\begin{array}{l}\text { Extract } \\
\text { solution }\end{array}$ & Extract with hexane, analyze the extract by LC-MS & $\begin{array}{l}\text { (Watson et al. 2004, } \\
\text { Polzin et al. 2009, } \\
\text { Ashley et al. 2010, } \\
\text { Brinkman et al. 2012) }\end{array}$ \\
\hline Terpenoids & Solanesol & $13190-97-1$ & 631.1 & $\begin{array}{l}\text { Cigarette } \\
\text { filter }\end{array}$ & $\begin{array}{l}\text { Extract } \\
\text { solution }\end{array}$ & Extract with $\mathrm{KOH}$, analyze the extract with LC-MS-MS & (Watson et al. 2004) \\
\hline Unknown & $\begin{array}{l}\text { 6-(2,6-Dichlorophenoxy) } \\
\text { Pyrimidine-2,4-Diamiine }^{d}\end{array}$ & & 271 & $\begin{array}{l}\text { Cigarette } \\
\text { butt }\end{array}$ & Water & $\begin{array}{l}\text { Leach into distilled water, analyze the leachate by LC- } \\
\text { MS }\end{array}$ & (Zhao et al. 2010b) \\
\hline
\end{tabular}

aMW: molecular weight

'Dedia: media describes the environment the detected chemicals were analyzed in (air, water, extract solution, cigarette filter, cigarette butt).

'The specific chemicals of tobacco specific nitrosamines (TSNAs) are not available in the cited paper (Verdolotti et al. 2012)

${ }^{d}$ CAS number can't be found based on the name and the structure reported in the reference (Zhao et al. 2010b), so it is classified as unknown chemical. 


\begin{tabular}{|c|c|c|}
\hline Acronym & Method & Description \\
\hline EM-MS & $\begin{array}{l}\text { electron monochromatic- } \\
\text { mass spectrometry }\end{array}$ & $\begin{array}{l}\text { Chemical species are ionized with electron ionization, and } \\
\text { then sorted based on their mass-to-charge ratio. The ion } \\
\text { signal as a function of the mass-to-charge ratio is } \\
\text { characteristic of different chemicals. }\end{array}$ \\
\hline FAAS & $\begin{array}{l}\text { flame atomic absorption } \\
\text { spectrometry }\end{array}$ & $\begin{array}{l}\text { A flame heats a sample, causing the atoms in the sample to } \\
\text { undergo a transition from the ground state to an excited } \\
\text { state. In the excited state the atoms absorb the light passed } \\
\text { through the sample. The elements in the sample are } \\
\text { identified based on the absorption of light by free atoms in } \\
\text { a gaseous state. }\end{array}$ \\
\hline GC-MS & $\begin{array}{l}\text { gas chromatography-mass } \\
\text { spectrometry }\end{array}$ & $\begin{array}{l}\text { A sample is forced by a gas through a column that is lined } \\
\text { with a stationary phase which causes the components to } \\
\text { separate. A mass spectrometer ionizes the chemical } \\
\text { compounds to generate charged molecules which are } \\
\text { separated according to their mass-to-charge ratio by } \\
\text { electromagnetic fields. The ion signal detected is then } \\
\text { processed into mass spectra. }\end{array}$ \\
\hline GC-FID & $\begin{array}{l}\text { gas chromatography-flame } \\
\text { ionization detector }\end{array}$ & $\begin{array}{l}\text { A sample is forced by a gas through a column that is lined } \\
\text { with a stationary phase which causes the components to } \\
\text { separate. A flame ionization detector ionizes the chemical } \\
\text { compounds producing a signal in proportion to the } \\
\text { concentration of organic species in a gas stream. }\end{array}$ \\
\hline HG-AFS & $\begin{array}{l}\text { hydride generation-atomic } \\
\text { fluorescence spectrometry }\end{array}$ & $\begin{array}{l}\text { A technique for speciation studies of hydride forming } \\
\text { elements (e.g. Arsenic) and Mercury. A hydride is a } \\
\text { compound that generally contains one or more hydrogens } \\
\text { with reducing properties. Atomic fluorescence } \\
\text { spectrometry is based on absorption of radiation by an } \\
\text { atomic vapor and subsequent emission of radiation at } \\
\text { wavelengths that are characteristic of the atomic species } \\
\text { present. }\end{array}$ \\
\hline HPLC-FLD & $\begin{array}{l}\text { high performance liquid } \\
\text { chromatography-fluorescence } \\
\text { detector }\end{array}$ & $\begin{array}{l}\text { A sample is forced by a liquid through a column that is } \\
\text { packed with a stationary phase which causes the } \\
\text { components to separate. Different components will adsorb } \\
\text { to the column at different rates, thereby separating the } \\
\text { components as they flow out of the column. Components } \\
\text { fluoresce in proportion to the concentration of the } \\
\text { component. }\end{array}$ \\
\hline HPLC-UV & $\begin{array}{l}\text { high performance liquid } \\
\text { chromatography-UV detector }\end{array}$ & $\begin{array}{l}\text { A sample is forced by a liquid through a column that is } \\
\text { packed with a stationary phase which causes the } \\
\text { components to separate. Different components will adsorb } \\
\text { to the column at different rates, thereby separating the }\end{array}$ \\
\hline
\end{tabular}




\begin{tabular}{|c|c|c|}
\hline & & $\begin{array}{l}\text { components as they flow out of the column. Components } \\
\text { absorb UV spectra in proportion to the concentration of the } \\
\text { component. }\end{array}$ \\
\hline ICP-AES & $\begin{array}{l}\text { inductively coupled plasma- } \\
\text { atomic emission spectroscopy }\end{array}$ & $\begin{array}{l}\text { A technique for elemental analysis that uses a plasma } \\
\text { source to dissociate the sample into its constituent atoms } \\
\text { or ions, exciting them to a higher energy level. As they } \\
\text { return to their ground state, they emit photons of a } \\
\text { characteristic wavelength depending on the element } \\
\text { present. This light can be recorded with an optical } \\
\text { spectrometer. }\end{array}$ \\
\hline ICP-OES & $\begin{array}{l}\text { inductively coupled plasma- } \\
\text { optical emission spectroscopy }\end{array}$ & $\begin{array}{l}\text { A technique for elemental analysis that uses a plasma } \\
\text { source to dissociate the sample into its constituent atoms } \\
\text { or ions, exciting them to a higher energy level. As they } \\
\text { return to their ground state, they emit photons of a } \\
\text { characteristic wavelength depending on the element } \\
\text { present. This light can be recorded with an optical } \\
\text { spectrometer. }\end{array}$ \\
\hline INAA: & $\begin{array}{l}\text { instrumental neutron } \\
\text { activation analysis }\end{array}$ & $\begin{array}{l}\text { A technique for elemental analysis that bombards the } \\
\text { sample with neutrons, causing the elements to form } \\
\text { radioactive isotopes. Based on the known radioactive } \\
\text { emissions of different elements, the emissions from the } \\
\text { sample can be analyzed to determine the concentration of } \\
\text { elements in it. }\end{array}$ \\
\hline LC-MS & $\begin{array}{l}\text { liquid chromatography-mass } \\
\text { spectrometry }\end{array}$ & $\begin{array}{l}\text { A sample is forced by a liquid through a column that is } \\
\text { packed with a stationary phase which causes the } \\
\text { components to separate. Different components will adsorb } \\
\text { to the column at different rates, thereby separating the } \\
\text { components as they flow out of the column. A mass } \\
\text { spectrometer ionizes the chemical compounds to generate } \\
\text { charged molecules which are separated according to their } \\
\text { mass-to-charge ratio by electromagnetic fields. The ion } \\
\text { signal detected is then processed into mass spectra. }\end{array}$ \\
\hline LC-MS-MS & $\begin{array}{l}\text { liquid chromatography-mass } \\
\text { spectrometry-mass } \\
\text { spectrometry }\end{array}$ & $\begin{array}{l}\text { A sample is forced by a liquid through a column that is } \\
\text { packed with a stationary phase which causes the } \\
\text { components to separate. Different components will adsorb } \\
\text { to the column at different rates, thereby separating the } \\
\text { components as they flow out of the column. In a mass } \\
\text { spectrometer, ions are produced and separated by their } \\
\text { mass-to-charge ratio. However, in mass spectrometry-mass } \\
\text { spectrometry (MS/MS), ions of particular mass-to-charge } \\
\text { ratios are further dissociated into fragment ions. These } \\
\text { fragment ions are then separated and detected in a second } \\
\text { stage of mass spectrometry. }\end{array}$ \\
\hline LSC & liquid scintillation counter & $\begin{array}{l}\text { A technique for detection and quantification of } \\
\text { radioactivity. A scintillation fluid is added to the sample }\end{array}$ \\
\hline
\end{tabular}




\begin{tabular}{|ll|}
\hline NMR $\quad$ nuclear magnetic resonance & $\begin{array}{l}\text { which interacts with the beta particles released from the } \\
\text { sample. The energized molecules of the scintillation fluid } \\
\text { emit photons of light at intensities that are proportional to } \\
\text { the energy of the beta particle. The emitted spectra are } \\
\text { unique for different-energy beta emitters. }\end{array}$ \\
& $\begin{array}{l}\text { An analytical technique used to determine the structure of } \\
\text { organic compounds. Nuclear magnetic resonance is based } \\
\text { on the absorption of electromagnetic radiation by a nucleus } \\
\text { having a magnetic moment when placed in an external } \\
\text { magnetic field. The intramolecular magnetic field around an } \\
\text { atom in a molecule changes the resonance frequency, } \\
\text { which provides details of the electronic structure of a } \\
\text { molecule. }\end{array}$ \\
\hline SPME & $\begin{array}{l}\text { A sampling technique that involves the use of a fiber coated } \\
\text { with a material which extracts both volatile and non- } \\
\text { volatile compounds from different kinds of media (liquid or } \\
\text { gas phase). }\end{array}$ \\
\hline
\end{tabular}

\subsubsection{Chemicals Emitted from Cigarette Butts into Air}

As mentioned before, no study has investigated the airborne emissions from cigarette butts in indoor or outdoor environmental conditions. However, there are four studies that measured the emissions from cigarette butts into the airborne headspace of test vials. In this section, we summarize the four studies. Both the measured chemicals and a brief description of the analytical methods are summarized in Table 2 and Table 3, respectively.

Fukuhara et al. (1985) detected a range of volatile components (e.g., carbonyls, hydrocarbons, pyrroles and terpenes) in the headspace of vessels that contained cigarette butts, and found that these compounds were not different from those found in mainstream smoke. They also found that the tar-like odors in butts may result from 2,3-pentanedione, $\mathrm{N}$-methyl pyrrole 3-methyl pyrrole, isocapronitrile, pyrrole, and 2-methyl pyrrole. You et al. (2014) developed a static headspace gas chromatography - flame ionization detector (GC-FID) method for quantifying menthol concentrations in the cigarette filter and cigarette papers (tipping and wrapping papers together) in a mentholated cigarette. The samples were first heated at $100^{\circ} \mathrm{C}$ for 30 minutes in a headspace sampler, and then measured by GC-FID. The methanol concentration in the cigarette papers ranged from $3.0 \mathrm{mg} / \mathrm{g}$ paper to $4.1 \mathrm{mg} / \mathrm{g}$ paper, while the methanol concentration in the cigarette filter was $13.7 \mathrm{mg} / \mathrm{g}$ paper to $27.2 \mathrm{mg} / \mathrm{g}$ paper. Huang et al. (2014) measured the emissions of cigarette tipping paper into the headspace of vials. The samples were first incubated at $80^{\circ} \mathrm{C}$ for $45 \mathrm{~min}$, and then 20 volatile organic compounds (VOCs) were quantified using gas chromatography-mass spectrometry (GC-MS). Among the 20 target VOCs, methanol, ethanol, isopropanol, ethyl acetate, 1-methoxy-2-propanol, and n-propyl acetate were the most abundant, with concentration ranging from $0.01 \mathrm{mg} / \mathrm{m}^{2}$ paper to $3.3 \mathrm{mg} / \mathrm{m}^{2}$ paper, $1.0 \mathrm{mg} / \mathrm{m}^{2}$ paper to 13.2 $\mathrm{mg} / \mathrm{m}^{2}$ paper, $0.0 \mathrm{mg} / \mathrm{m}^{2}$ paper to $8.9 \mathrm{mg} / \mathrm{m}^{2}$ paper, $0.03 \mathrm{mg} / \mathrm{m}^{2}$ paper to $1.33 \mathrm{mg} / \mathrm{m}^{2}$ paper, 0.0 $\mathrm{mg} / \mathrm{m}^{2}$ paper to $3.0 \mathrm{mg} / \mathrm{m}^{2}$ paper, and $0.02 \mathrm{mg} / \mathrm{m}^{2}$ paper to $20.7 \mathrm{mg} / \mathrm{m}^{2}$ paper, respectively. A recent study developed a static headspace GC-MS method to quantify benzene, toluene, $\mathrm{m}$ xylene, $\mathrm{p}$-xylene, o-xylene, styrene and ethylbenzene concentrations in cigarette filters ( $\mathrm{Ji}$ et al. 2015). The method heated the cigarette filters at $120^{\circ} \mathrm{C}$ for $30 \mathrm{~min}$ in the headspace vials. The 
measured concentrations for benzene, toluene, ethylbenzene, $p$-xylene, $m$-xylene, o-xylene, and styrene were from $0.4 \mu \mathrm{g} /$ filter to $1.1 \mu \mathrm{g} /$ filter, $1.1 \mu \mathrm{g} /$ filter to $4.7 \mu \mathrm{g} /$ filter, $0.4 \mu \mathrm{g} /$ filter to 1.7 $\mu \mathrm{g} /$ filter, $0.3 \mu \mathrm{g} /$ filter to $1.4 \mu \mathrm{g} /$ filter, $0.8 \mu \mathrm{g} /$ filter to $3.3 \mu \mathrm{g} /$ filter, $0.4 \mu \mathrm{g} /$ filter to $1.4 \mu \mathrm{g} /$ filter, and $2.0 \mu \mathrm{g} /$ filter to $5.7 \mu \mathrm{g} /$ filter, respectively.

These four studies provide some basis for understanding the potential airborne emissions into indoor or outdoor environments. However, the study reported by Fukuhara et al. (1985) was limited in the number of cigarette butts tested and quantification of the identified chemicals. For the three more recent studies (Huang et al. 2014, You et al. 2014, Ji et al. 2015), the types of chemicals detected are very limited and only cigarette filters or tipping papers were measured. These studies did not include the unburned tobacco or ash of cigarette butts, which likely emit different chemicals from the filters and tipping papers. In addition, the high incubation temperatures $\left(>80^{\circ} \mathrm{C}\right)$ will enhance the emission of some non-volatile chemicals that may not emit to a significant degree at normal indoor and outdoor temperatures. These testing conditions could result in overloading the GC-MS if the unburned tobacco and ash were included. Finally, all of these studies only measured freshly generated cigarette butts, or part of the unburned cigarette, and didn't examine the influence of several factors that could impact the emission of cigarette butts, such as the exposed temperature, relative humidity, aging, and ultra-violet (UV) radiation.

\subsubsection{Chemicals Emitted from Cigarette Butts into Water}

In this section, we summarize the chemicals found in studies on emissions from cigarette butts or their components into water. Both the chemicals detected and more detailed information on the measurement methods are shown in in Table 2 and Table 3, respectively.

Compounds within cigarette butts can leach out into water, potentially threatening human health and the environment, especially marine ecosystems (Kadir and Sarani 2015). Zhao et al. (2010a) measured the chemicals in both aerobic and anaerobic cigarette butt water extracts. They found that the main chemicals in both water extracts were aromatic amines, e.g., nicotine and $\mathrm{N}$-nitroso-nornicotine (NNN). More types of compounds were found in aerobic water extracts than anaerobic water extracts, which may be because of the oxidization of amine compounds in aerobic water. Another study published by Zhao et al (2010b) also primarily detected amines in water, but also found different chemicals than the first Zhao et al. (2010a) study, e.g., cotinine.

Emissions from cigarette butts into water can be a fast process. Standardized cigarette butts produced with a smoking machine leached $7.3 \mathrm{mg}$ of nicotine per $\mathrm{g}$ of butts into $1 \mathrm{~L}$ of purified water, of which $50 \%$ was released in the first 27 minutes during the experiment (Green et al. 2014). The same study also found that the cumulative nicotine release from fifteen consecutive rainfall events with $1.4 \mathrm{~mm}$ of precipitation for each event was $3.8 \mathrm{mg}$ of nicotine per $\mathrm{g}$ butt, of which $47 \%$ was released during the first event (Green et al. 2014). A study of metals (aluminum, barium, chromium, cadmium, copper, iron, lead, manganese, nickel, strontium, tin and zinc) showed that more than half the mass was leached within one day from the cigarette butts into acidic aqueous solution (Moerman and Potts 2011).

Interestingly, cigarette butts can not only emit chemicals into water, but also absorb chemicals from water. Chen et al. (2012) used cigarette filters as adsorbents for pre-concentration of fluoroquinolones antibacterial agent. Hence, it is possible that not all chemicals present in the cigarette butt as it ages are from the original cigarette or the result of the smoking process. 


\subsubsection{Chemicals Measured in Cigarette Butts}

Since there are limited data on chemicals emitted from cigarette butts into air or water, we examined the literature for information on chemicals that are present in cigarette butts.

In this section, we summarize the chemicals from studies on extraction of cigarette butts or individual butt components by solvents. We also examine studies that directly analyzed the cigarette butts or individual butt components via their radioactivity. Chemicals detected in cigarette butts and more detailed information on the measurement methods are included in Table 2 and Table 3, respectively.

\subsubsection{Metals}

Metals are commonly detected within cigarette butts or individual butt components (Wu et al. 1997, Huang et al. 2013). Cadmium, copper, manganese and zinc were measured in cigarette butts at concentrations up to $104 \mu \mathrm{g} / \mathrm{g}$ butt (Pelit et al. 2013). Mercury and arsenic concentrations in cigarette filters have been measured between $0.002 \mathrm{mg} / \mathrm{kg}$ to 0.051 $\mathrm{mg} / \mathrm{kg}$ and $0.025 \mathrm{mg} / \mathrm{kg}$ to $0.651 \mathrm{mg} / \mathrm{kg}$, respectively (Wang et al. 2007). Zinc and iron have been detected in the cigarette tobacco, filter and ash (Wang and Finlayson-Pitts 2003). After smoking, $15 \%$ to $50 \%$ of heavy metals (lead, arsenic, cobalt, cadmium, potassium, antimony and zinc) originally in cigarettes is retained in cigarette butts (Galazyn-Sidorczuk et al. 2008, Wu et al. 2012, Carolina Talio et al. 2013). However, despite the detection of mercury in the cigarette butts, a majority of mercury that is initially present in the cigarette was released into the air during smoking and not trapped on cellulose acetate filters (Kowalski and Wiercinski 2009).

\subsubsection{Polycyclic Aromatic Hydrocarbons (PAHs) and Terpenoids}

Since cigarette butt samples can be collected easily and noninvasively, levels of some chemicals in cigarette butts are often used to estimate mouth-level intake of the chemicals for smokers. Benzo[a]pyrene (B[a]P) levels in used cigarette filters have been measured and demonstrated to be highly correlated with $\mathrm{B}[\mathrm{a}] \mathrm{P}$ measured in mainstream smoke. This correlation allows $\mathrm{B}[\mathrm{a}] \mathrm{P}$ concentrations in cigarette butts to be used as an estimate of mouth-level $B$ [a]P exposure of smokers (Ding et al. 2014, Zhang et al. 2014). PAHs in cigarette filters have been studied using solid-phase microextraction (SPME) and high performance liquid chromatography (HPLC) (Demirci 2014). The sum all of the studied PAHs found in the filter tar of three brands of cigarettes were $17.9 \mathrm{ng}, 66.7 \mathrm{ng}$ and $320.2 \mathrm{ng}$ per cigarette.

Solanesol is a chemical found in tobacco leaves. The concentration solanesol deposited in cigarette filters has been shown to be stable for over four weeks after smoking (Watson et al. 2004). As a result, solanesol extracted from cigarette filters may be used as an indicator chemical to assess smokers' mainstream smoke exposure to tobacco specific nitrosamines (TSNAs) (Polzin et al. 2009), tar (Watson et al. 2004) and nicotine (Watson et al. 2004, Polzin et al. 2009). The concentrations of solanesol in cigarette filters were used to estimate the mouth-level intake of 4-(methylnitrosamino)-1-(3-pyridyl)-1-butanone (NNK) (Ashley et al. 2010, Brinkman et al. 2012), which was found to be significantly associated with urinary concentrations of its major metabolite 4-(methylnitrosamino)-1-(3-pyridyl)-1-butanol (NNAL) (Ashley et al. 2010).

\subsubsection{Insecticides and Plant Growth Regulators}

Different chemicals are often used to protect tobacco plants prior to harvest. These chemicals can be in the unburned tobacco of a cigarette butt or potentially transferred to 
other parts of the cigarette butt and detected in the extract of cigarette butts. Maleic hydrazide (1,2-dihydro-3,6-pyridazinedione), a plant growth regulator that inhibits the growth of suckers on tobacco plants, is often present in cigarettes, ranging from $1 \mu \mathrm{g} / \mathrm{g}$ cigarette to $100 \mathrm{\mu g} / \mathrm{g}$ cigarette (Zhang et al. 2012). Zhang et al. (2012) found that maleic hydrazide can be transferred to the cigarette butt in the range from $1.1 \%$ to $1.9 \%$ of the initial mass present in the cigarette. Chlorantraniliprole is the active ingredient in the insecticide used to control budworm and hornworm in tobacco plants. A study on pyrolysis of ${ }^{14} \mathrm{C}$ chlorantraniliprole in a cigarette showed that about $17 \%$ of spiked ${ }^{14} \mathrm{C}$ chlorantraniliprole was found in extracts of cigarette butts after smoking (Gaddamidi et al. 2011). Of the spiked radio-labelled insecticide imidacloprid [1-(6-chloropyridin-3-ylmethyl)$\mathrm{N}$-nitro-imidazolidin-2-ylideneamine] in tobacco leaves, $39 \%$ of the radioactivity was found in cigarette butts after the smoking of cigarettes made of the spiked leaves (Clark et al. 1998).

\subsubsection{Other Organics}

In addition to the aforementioned chemicals, phenolic compounds, tobacco specific nitrosamines (TSNAs), carbonyls, phthalates and nitropolycylic aromatic hydrocarbons (NPAHs) have also been detected in cigarette butts.

Sonication solvent extraction of cigarette filters followed by HPLC analysis has been used to measure seven major phenolic compounds (hydroquinol, resorcinol, catechol, phenol, pcresol, $\mathrm{m}$-cresol and o-cresol) on cigarette filters in a comparative study run between six laboratories (Hu et al. 2015). Rutin, a kind of phenolic compound, was found in cigarette filters at a mass of $0.1 \mu \mathrm{g} /$ filter to $0.3 \mu \mathrm{g} /$ filter (Sun et al. 2012). However, the rutin mass in the cigarette filter was two orders of magnitude lower than in the tobacco part of the cigarette (Sun et al. 2012), and rutin is likely not volatile enough (molecular mass $610.5 \mathrm{~g}$ $\mathrm{mol}^{-1}$, boiling point $956^{\circ} \mathrm{C}$ ) to be emitted into the air at significant levels from extinguished butts.

TSNAs have been detected in cigarette filters by extracting the filters with a mixture of deuterated water/dimethyl sulfoxide and then analyzing with nuclear magnetic resonance (NMR) (Verdolotti et al. 2012).

Yu et al. (2013) extracted carbonyls (formaldehyde, acetaldehyde, acetone, acrolein, propionaldehyde, crotonaldehyde, 2-butanone and butyraldehyde) from cigarette filters and determined that the filtration efficiencies of four different types of cigarette filters (i.e. cellulose acetate filter, activated carbon filter, polypropylene fiber filter, and paper filter) were roughly $5 \%$ for the total content of the eight carbonyls.

Phthalate esters were extracted with water and ethanol from 45 cigarette filters and analyzed using GC-MS (Zhang et al. 2012). Among the 17 targeted phthalate esters, only dibutyl phthalate (DBP), diisobutyl phthalate (DIBP) and di(2-ethylhexyl)phthalate (DEHP) were found in the cigarette filters with detection frequency for each phthalate less than 20 $\%$ (Zhang et al. 2012).

NPAHs in cigarette filters have been measured using toluene extraction followed by electron monochromator-mass spectrometry (EM-MS) (John Dane et al. 2002).

\subsubsection{Mainstream and Sidestream Smoke Chemicals Potentially in Cigarette Butts}

The previous sections of this report reviewed chemicals that were present in a cigarette butt or emitted into air or water from a cigarette butt. In addition to the chemicals identified in cigarette 
butts (Table 2), chemicals found in cigarette smoke may also be present in cigarette butts despite not having been identified as such in a previous study. Hence, the literature on chemicals in cigarette smoke was reviewed to guide the planning process for investigating airborne emissions from cigarette butts by (1) more broadly identifying the types of compounds that may be emitted from cigarette butts into the air, and (2) identifying a wider range of the analytical tools available. Preliminary experiments (section 4.2.5) show that many of the components in cigarette smoke are similar to the components measured in air above a freshly-smoked, extinguished cigarette butt and an aged cigarette butt.

Cigarette smoke is a complex mixture of 40000 to 100000 compounds (Dalluge et al. 2002) including metals, hydrocarbons, phenols, quinones, alcohols, phytosterols, aldehydes, ketones, carboxylic acids, ethers, carbohydrates, esters, anhydrides, amines, amides, nitriles, inorganic gases (e.g. $\mathrm{CO}_{2}, \mathrm{CO}, \mathrm{NO}_{x}$ ), free radicals and other compounds. Most of the organic compounds are in the range of $n$-heptane to $n$-dodecane. Cigarette smoke is comprised of mainstream and sidestream smoke. Mainstream smoke is the term used for smoke drawn through "the butt end of the cigarette during the smoking process" (ISO3308 2012) and can also be applied to the smoke that a smoker exhales. Sidestream smoke refers to the smoke that wafts off the lit end of the cigarette during the smoking process (ISO3308 2012). Sidestream smoke makes up about $85 \%$ of environmental tobacco smoke (Pieraccini et al. 2008). Mainstream smoke includes several chemicals classified by the International Agency Research On Cancer (IARC) as Group 1 Human Carcinogens: benzene, cadmium, arsenic, nickel, chromium, 2-naphthyl-amine, vinyl chloride, 4aminobiphenyl and beryllium (Smith et al. 1997). Sidestream smoke contains higher concentrations of some chemicals than mainstream smoke due to incomplete combustion. The chemical composition of sidestream and mainstream smoke is also different, because the combustion temperature of a smoldering cigarette is much lower than the temperature during a puff. For instance, aromatic amines (Luceri et al. 1993) and PAHs (Lodovici et al. 2004) are found at higher levels in sidestream smoke than in mainstream smoke as a result of incomplete combustion as cigarettes smolder.

The sample collection methods for chemicals in cigarette smoke are based on their inherent stability or some aspect of their chemical reactivity (Liu et al. 2011). There is greater diversity in the trapping and analysis techniques used for volatile compounds in smoke than in the extraction techniques of cigarette butts presented in the previous section. Compounds such as tar, nicotine, phenols, tobacco-specific nitrosamines (TSNA) and benzo[a]pyrene are typically collected onto glass fiber filters. Aromatic amines, hydrogen cyanide, ammonia, certain semi-volatiles, and certain carbonyls and volatile compounds are often trapped using impingers or glass fiber filters. Other trapping techniques include sorbent tubes (for certain semi-volatiles), activated silica gel (for hydrogen cyanide and certain carbonyls) and DNPH treated filters (for certain carbonyls). Solid sorbent trapping and thermal desorption-gas chromatography has been used extensively over the last few decades to measure gas phase and particulate matter components in cigarette smoke (Higgins et al. 1984). Inorganic gases, such as carbon monoxide and nitric oxide, are sampled using gas collection bags.

Real-time smoke analytical techniques have highlighted the dynamic and reactive nature of some of the compounds in cigarette smoke (Liu et al. 2011). Boldridge and Ingebrethsen (1986) developed a model to predict the changes in cigarette smoke aerosol as it travels through a length of tobacco-filled rod.

Compounds in cigarette smoke that can readily volatilize are more likely to appear in airborne emissions from cigarette butts. Compounds with high boiling points (e.g. metals, compounds with 
high molecular weights) are unlikely to get volatilized from cigarette butts. Sections 3.3.4.1 and 3.3.4.2 categorize compounds in cigarette smoke into these two classes and discuss the analytical techniques used to measure these compounds. These sections do not attempt to be an authoritative or exhaustive analysis of compounds in mainstream or sidestream smoke. Rather, they provide a background on analytical techniques in order to support the discussions of experimental investigations described in Section 4.

\subsubsection{Chemicals in Cigarette Smoke Unlikely to be Present in Airborne Emissions from Cigarette Butts}

Only some compounds from cigarette smoke that are retained on the discarded cigarette butt are likely to emit into the air. Some compounds can be too volatile to get fully retained in the cigarette filter, and others can get retained but are very unlikely to be emitted from cigarette butts because they are not volatile enough. Petraru et al. (2013) examined the ability of 29 different cigarette filters to retain chemicals, showing that lower molecular weight chemicals breakthrough filters faster. These lower molecular weight chemicals included methane, ethane, propane, 2-methylpropane, ethene, acetylene, 1-butene, 1,3 butadiene, 2-butene, cis-2-butene, 2-methyl-2-butene, benzene, toluene, furan, 2methylfuran, 2,5 meththylfuran, acetaldehyde, acrolein, propionaldehyde, 2methylpropanal, crotonaldehyde, acetone, methyl vinyl ketone, methyl ethyl ketone, acetonitrile, propionitrile, methyl acrylate, methanol and chloromethane. Other compounds may get retained in cigarette butts but are unlikely to volatilize from cigarette butts at significant rates if their boiling points are high. These compounds can, however, leach out of the discarded cigarette butt into water, soil, and other media, as described in section 3.3.2. Analytical techniques and measurements of these compounds in cigarette smoke is described below.

\subsection{Metals}

Most particulate phase compounds emitted in cigarette smoke are collected with glass fiber filters (Cambridge Filter Pads) and analyzed using a range of extraction solvents and analytical techniques (Liu et al. 2011). Metals present in cigarette smoke are typically associated with particles. Hence, most airborne metal studies collect particulate matter on filters and measure metals on the filter using inductively-coupled plasma mass spectrometry (ICP-MS) or graphite furnace atomic absorption spectrometry (GFAAS). Wagner et al. (2001) measured arsenic, cadmium and lead in this manner. Due to the association with particles, it is unlikely that metals will emit into the air from cigarette butts.

Although most metal emissions are typically associated with particles, there is some potential for organometallic compounds to be present and volatilize. The emission of nickel tetracarbonyl from cigarettes was investigated by Torjussen et al. (2003). Nickel tetracarbonyl has a boiling point of $43^{\circ} \mathrm{C}$, and hence it could be emitted from a butt if present.

\subsection{Tobacco-Specific Nitrosamines}

Tobacco-specific nitrosamines (TSNAs) are carcinogens (Sleiman et al. 2009, Verdolotti et al. 2012), and TSNA urinary metabolites can be detected after secondhand smoke exposure (Schick et al. 2013). TSNAs are typically associated with particles and sorbed to indoor surfaces (Schick et al. 2014). TSNAs have been analyzed by trapping particles on glass fiber filters and extracting them using various methods including supercritical fluid extraction (carbon dioixide with $10 \%$ methanol) (Brunnemann et al. 1996, Sleiman 
et al. 2009). The elution is then analyzed using gas chromatography-ion-trap tandem mass spectrometry.

Emission of TSNAs vary over an order of magnitude between different cigarettes. Analyzing secondhand smoke particles, Brunnemann et al. (1996) found $\mathrm{N}$-nitrosonornicotine (NNN) from $28 \mathrm{ng}$ to $730 \mathrm{ng}$ per cigarette, and for 4-(methylnitrosamino)-1-(3-pyridyl-1-butanone (NNK) from 16 ng to 369 ng per cigarette depending on the cigarette composistion. The TSNA concentrations correlated with nicotine concentrations. Haorah et al. (2001) measured N-nitroso compounds and their precursors in cigarette smoke using sampling filters to collect the smoke. The method employed measured the total amount of $\mathrm{N}$-nitroso compounds present, not individual species, as is possible with GC-MS methods. The total amount of N-nitroso compounds was found to be more than an order of magnitude higher than previously published values of TSNAs, including NNN and NNK, indicating that most of the Nnitroso compounds do not consist of these nitrosamines. The concentrations of precursors of $\mathrm{N}$-nitroso compounds correlated with particulate and tar concentrations.

All known TSNAs methods require the TSNA to be located in a solid phase (on particles or filters). Hence, since TSNAs are primarily seen associated with particles, they are unlikely to be present when no particles are generated, as with cigarette butts.

\subsection{Carbon Monoxide}

Yang et al. (2015) analyzed carbon monoxide in mainstream cigarette smoke from four different types of cigarettes using tunable diode laser absorption spectroscopy (TDLAS) with a modified single port smoking machine. Carbon monoxide is a very volatile gas that is generated due to incomplete combustion. It is therefore not expected to persist at significant concentrations in cigarette butts.

\subsubsection{Chemicals in Cigarette Smoke that may be Present in Airborne Emissions from Cigarette Butts}

Compounds with moderate volatility in cigarette smoke that do get retained in the cigarette butt can potentially be emitted from a cigarette butt after it is disposed, depending on the environmental conditions (temperature, relative humidity, UV intensity, etc.) to which it was exposed. Analytical techniques and measurements of these types of compounds are described below.

\subsection{Nicotine}

Nicotine is more volatile than most TSNAs, making a wider range of analytical methods available, in addition to extraction from particles on particle collection filters. Ishizu et al. (2013) used a passive XAD-4/PDMS (styrene-divinylbenzene copolymer with polydimethylsiloxane) coated glass fiber filter (extracted and analyzed using GC-MS) to show that the ratio of nicotine to 3-ethenylpyridine decreased as the secondhand smoke aged. Watson et al. (2004) analyzed nicotine concentrations using SPME followed by GC-MS. Fu et al. (2013) measured nicotine in secondhand smoke using sodium bisulphate-treated filters and GC-MS.

Nicotine can exist in three forms: 1) a diprotonated molecule that exists when in contact with envrionments with $\mathrm{pH}<3,2$ ) a monoprotonated molecule that can exist in a tobacco filter (where $5<\mathrm{pH}<6$ ), and 3 ) the free-base molecule (Liang and Pankow 1996, Ishizu and Ishizu 2013). Only the free-base species is volatile (Liang and Pankow 1996). Free-base nicotine is thought to be the dominant species in cigarette smoke 
because cigarette smoke is generally alkaline. Adding ammonia-releasing chemicals to the tobacco can ensure the alkaline nature of the smoke, pushing more of the nicotine into the free-base form (Liang and Pankow 1996). Free-base nicotine is also the dominant form of nicotine on the cigarette filter when freshly smoked, although the $\mathrm{pH}$ and nicotine species can change with environmental exposures. Free-base nicotine is lipophilic and more readily absorbed across membranes, increasing its bioavailability. Watson et al. (2004) showed that fewer ventilation holes blocked in the filter resulted in a greater fraction of free-based nicotine in the mainstream smoke particulate matter.

\subsection{PAHs}

Several studies have measured PAHs in cigarette smoke. The sampling technique used most often is to collect sidestream smoke onto glass fiber filters, extract the samples with a solvent, and analyze the extracts using HPLC or GC-MS. Two- and three-ring PAHs are relatively volatile, which makes them likely to desorb from discarded cigarette butts and be emitted to the surrounding air. Five and six-ring PAHs (benzo[a]pyrene) are less volatile and less likely to be emitted to the surrounding air at measurable rates.

Endo et al. (2000) analyzed seven PAHs (including benzo[a]pyrene) collected on filters, which were extracted with ethanol/benzene and analyzed on HPLC. Demirci and Alver (2013) analyzed PAHs using hollow, fiber-liquid-phase microextraction and HPLC-UV. Lodovici et al. (2004) measured PAHs in mainstream and sidestream smoke from 14 commercial brands of cigarettes using HPLC. PAH concentrations in mainstream smoke varied by three-fold for the different brands, and were ten times higher in sidestream smoke compared to mainstream. They also found that most tobacco-related PAHs occurred in the particulate phase.

Daher et al. (2010) captured particulate PAHs from sidestream smoke on glass fiber filters and analyzed them using GC-MS. Xie et al. (2012) analyzed 14 PAHs that were collected on filters, extracted, cleaned with a silicon cartridge, concentrated, and finally injected into a GC-MS. Liang et al. (1996) captured secondhand smoke particles on filters and measured the partitioning coefficient between particle phase and gas phase for PAHs (fluorene, phenathrene, flouranthene, pyrene and chrysene) and nitrogencontaining PAHs (quinoline and isoquinoline) using thermal desorption tubes and GCMS. This study highlights that lighter PAHs will likely be present in the air phase near cigarette butts.

Schick et al. (2014) studied the effects of aging on the concentration of 16 PAHs, nicotine, cotinine and TSNAs in cigarette smoke as it was passed through a flow reactor at 1 air change per hour to simulate residential air change rates when a mechanical ventilation system is on. Measurements were made with GC-MS and showed that the levels of PAHs, nicotine and TSNAs were reduced by $60 \%$ to $80 \%$ over a 60 -minute period, but the levels of cotinine did not decrease over this period. Other studies in controlled indoor environments have also shown that nicotine is removed faster than gas-phase hydrocarbons and some particulate species (Eatough 1990). Schick et al. also measured sorption and deposition of nicotine, cotinine and TSNAs on materials placed in a flow reactor. They concluded that the majority of these compounds deposit on room surfaces when cigarettes are smoked in indoor environments, which can lead to the accumulation of carcinogens on indoor surfaces. Gradual desorption of these chemicals and their reaction with common indoor pollutants leads to chemical being present for extended periods of time after a cigarette has been smoked. 


\subsection{Other Organics}

Other organic gases have been studied using unique analytical techniques. Zheng et al. (2014) identified 67 gas-phase components in mainstream cigarette smoke using sorption tubes containing 3 beds of adsorbents (Carbopack C, Carbopack B and Carboxen III) analyzed using thermal desorption gas chromatography mass spectrometry (TD-GC-MS). Hertz-Schunemann et al. (2015) studied the evolution of organic gases during a two-second cigarette puff using a novel microprobe to extract gases from inside a burning cigarette and analyzed them with a photoionization mass spectrometer. The concentrations of various pyrolysis and combustion products such as 1,3-butadiene, toluene, acetaldehyde and phenol were monitored at different sampling locations within cigarettes. The measurements were combined to generate time-resolved concentration maps, showing the formation and destruction zones of the investigated compounds in the burning cigarette.

Reactive oxygenated species (ROS) are a broad class of chemicals that are reactive and contain oxygen. Cigarette filters have been shown to remove $26 \%$ of the gas-phase ROS and $17 \%$ of particle phase ROS (Zhao and Hopke 2012). However, gas-phase ROS is only a small fraction of total ROS (Zhao and Hopke 2012). Hence, volatile ROS may be present in cigarette butts, but likely not in large quantities.

\subsection{Hydrogen Cyanide}

Hydrogen cyanide has been measured in cigarette smoke using continuous flow analysis (Guo et al. 2015). Liu et al. (2014) noted that nitrogen oxides in cigarette smoke can dissolve in the alkaline solution used for the measurement of hydrogen cyanide in cigarette smoke, and can produce nitrates and nitrites, which further react with cyanide and result in underestimating the yield of hydrogen cyanide. They improved upon this method by collecting hydrogen cyanide on a Cambridge filter pad treated with a solution of sodium hydroxide and then analyzing it with a continuous flow analyzer containing isonicotinic acid and 1,3-dimethylbarbituric acid. Compared to the conventional method, this method was shown to be simpler, and the collected hydrogen cyanide had greater stability.

\subsection{Factors that Potentially Influence Airborne Emissions from Cigarette Butts}

There are a wide range of factors that can impact the airborne chemical emissions from cigarette butts. These include the brand of the cigarette, the length of the cigarette butt, the temperature during the aging process, the airflow around the butt, the airflow through the butt, the number of puffs taken while the cigarette was being smoked, the degradation of the cigarette butt, and if the butt was smoked by a machine or human. The following section highlights the studies that have looked at these factors.

\subsubsection{Brand}

The chemical content of cigarettes varies with the cigarette brand and the source of the tobacco, and each cigarette brand is engineered to have different performance. As a result, cigarette butts from various brands will have different emission characteristics. Demirci et al. (2013) was able to differentiate between brands by extracting cellulose acetate cigarette filters (with acetonitrile/nhexane) and analyzing them with HPLC-UV.

In a 2008 study, tar yield data were collected for 172 cigarettes sold in the United States, Canada, Australia, and the United Kingdom. A total of 11 cigarette design parameters were measured, including filter ventilation, cigarette pressure drop, filter pressure drop, tobacco rod length, filter length, cigarette diameter, tipping paper length, tobacco weight, filter weight, rod density and 
filter density. Filter ventilation was found to be the main design parameter that accounted for the tar yield differences among different brands (O'Connor et al. 2008).

Cigarettes of different brands use different types of tobacco. Ding et al. (2008) demonstrated nitrate contents are different in different types of tobacco, which can influence the levels of nicotine, TSNAs and PAHs in mainstream smoke.

Jenkins et al. (1979a, 1979b) showed that the tar, nicotine and carbon monoxide content of smoke varies between samples of a given brand of cigarettes, depending on the nation in which they are purchased. Djordjevic et al. (1991) compared cigarettes from the former Union of Soviet Socialist Republics (USSR) with those from the United States and Western Europe, and found that the nicotine level and the total alkaloid content of the cigarettes from the western countries were slightly higher than in the cigarettes from the former USSR. They observed that non-filter and filter cigarettes from the former USSR had slower burning rates and yielded 14 to 16.7 puffs per cigarette, while cigarettes in the western countries yielded 11 puffs per cigarette. Tar, benzo[a)pyrene and nitrosamines yields were reported to be especially high in the smoke of cigarettes with filters.

However, not all studies have shown a large difference between cigarette brands. Pieraccini et al. (2008) developed a SPME-GC-MS method to determine the components of mainstream and sidestream smoke for 15 different cigarette brands. The quantitative compositions of mainstream and sidestream smoke for all brands of cigarettes were similar, with 67 chemicals identified. For the quantified chemicals (benzene, toluene, $\mathrm{p}$-xylene, $\mathrm{m}$-xylene, pyridine, o-xylene, limonene, naphthalene, phenol and nicotine), the concentration difference among different brands was within an order of magnitude for both mainstream and sidestream smoke.

\subsubsection{Filter Material}

Cigarette filters are typically constructed from cellulose acetate, but the filter can be designed in each brand to enhance flavor properties and reduce toxic compounds in the mainstream smoke. While filter additives can reduce chemical concentrations in mainstream smoke (i.e., nitric oxide, carbon monoxide, hydrogen peroxide, aldehydes, vinyl chloride, 1,3-butadiene, isoprene, acrylonitrile, benzene, toluene and nitroso-compounds) (Smith et al. 1997, Valavanidis and Haralambous 2001, Liu et al. 2013, Awji et al. 2015), they can also increase the chemicals present in the filter that can emit later from the cigarette butt.

Each chemical may behave differently in the cigarette filter. Qin et al. (2014) studied the retention of crotonaldehyde in cigarette filters. Longitudinal concentration distribution of crotonaldehyde in the cigarette filter was found to be different from the distribution of nicotine, phenol and other smoke components.

Several studies have been conducted on reducing compounds in cigarette smoke by making modifications to cigarette filters (Dittrich et al. 2014, Duan et al. 2014, Chen et al. 2015, Marcilla et al. 2015). The filter modification can be engineered from a chemical or mechanical perspective. For instance, a study on the effect of cellulose acetate and polypropylene filter materials on semi volatiles in smoke indicated that the polar cellulose acetate filter selectively retains polar compounds, such as phenols and furans, while the non-polar polypropylene filter shows a nonselective retention behavior (Formella et al. 1992). Grooves can be added to cigarette filters to change the inertial impaction of particles and diffusion of chemicals. Duan et al. (2014) studied the effects of 35 kinds of grooved filters on the yield of particles, tar, nicotine and carbon monoxide in mainstream cigarette smoke. They found that increasing the length of the grooved section in the 
filter reduced the yields (up to a length of $20 \mathrm{~mm}$ after which filtration efficiency of particles and nicotine decreased), while the position of the grooved section did not influence the yields.

\subsubsection{Butt Length}

The length of the cigarette butt has been shown to impact the chemicals present in the cigarette butt. A longer cigarette butt will have more unburned tobacco and greater tobacco associated emissions than a shorter cigarette butt. Green et al (2014) reported that nicotine released into water increases as the butt length increases, as more nicotine is retained in tobacco than in the filter of cigarette butts. In contrast, the shorter cigarette butt will have a greater proportion of pyrolysis products and metals present.

Cigarettes can either be extinguished by the smoker or be self-extinguished. . There is no defined standard cigarette butt length for testing emissions. However, Watson et al. (2004) used a butt length of $23 \mathrm{~mm}$ or the length of the filter overlap plus $3 \mathrm{~mm}$, whichever was longer, for their research.

\subsubsection{Temperature}

Cigarette butts discarded outdoors are in a dynamic environment with a changing temperature, which can impact the chemicals present in the butt over time. Clayton et al. (2010) showed that nitrosamines levels in cigarette butts decreased after three weeks of storage at $22{ }^{\circ} \mathrm{C}$, especially for 4-(methylnitrosamino)-1-(3-pyridyl)-1-butanone (NNK). However, the nitrosamines levels in cigarette butts were relatively consistent over four weeks of storage at $-17^{\circ} \mathrm{C}$ and $4{ }^{\circ} \mathrm{C}$.

\subsubsection{Cross flow}

The cigarette burning process can be influenced by environmental conditions, which in turn can influence the components retained in the cigarette butt post-smoking. Modeling of combustion of a cigarette has indicated that increasing the ambient cross flow surrounding the cigarette increases the burn rate and temperature in the cigarette column (Saidi et al. 2006, Saidi et al. 2007). Different combustion temperatures will result in the formation of different pyrolysis products that can eventually deposit in the cigarette butt.

\subsubsection{Ventilation}

Ventilation through the cigarette during smoking can also influence the chemicals present. The burn rate, temperature and delivery of chemicals through a burning cigarette to the cigarette filter increases as a result of the increasing of ventilation through the cigarette (Saidi et al. 2006, Saidi et al. 2007). The change to these parameters can then result in the formation of different pyrolysis products. Dittrich et al. (2014) placed a band of porous paper in the center of the filter to minimize the loss of filter ventilation that occurs at the high flow rates encountered during human-smoking. This "split-tipping" facilitates the diffusional loss of volatile chemicals. Mouth level exposure to tar and nicotine was also lower in low-tar split-tipped cigarettes.

\subsubsection{Puff Number}

The number of puffs that a smoker draws through a cigarette has been shown to impact the chemicals present. Li et al. (2002) found that formaldehyde concentrations in mainstream smoke were higher in the first puff compared to subsequent puffs. The authors concluded that the heat exposure of the tobacco from previous puffs was the largest factor in differing formaldehyde concentrations. Li et al. (2002) also showed that heat treatment of cigarettes $\left(200^{\circ} \mathrm{C}\right.$ to $\left.250^{\circ} \mathrm{C}\right)$ prior to lighting reduced formaldehyde concentration by $20 \%$ to $90 \%$. This was attributed to the conversion of cellulose to other products at temperatures below $500^{\circ} \mathrm{C}$. 


\subsubsection{Degradation}

Cigarette butts will eventually degrade due to microbial degradation, photo-oxidation, mechanical abrasion and chemical degradation. However, not all components of cigarette butts are readily biologically degradable or photo-oxidized. Filters typically contain plasticized cellulose acetate fibers that are slow to degrade (Puls et al. 2011). Cellulose can be degraded by organisms with cellulose enzymes, but the acetyl groups in cellulose acetate first need to be removed to a certain extent (via the action of esterases or by chemical hydrolysis) for biodegradation of the cellulose backbone to initiate. Cellulose acetate can be photochemically degraded by UV wavelengths shorter than $280 \mathrm{~nm}$, but has limited photo-degradability in sunlight due to the lack of chromophores for absorbing UV light (Puls et al. 2011). Cellulose acetate cigarette butts tend to linger in the environment for extended periods of time for these reasons. After two years of outdoor decomposition, ${ }^{13} \mathrm{C}$-cross-polarization magic angle spinning (CPMAS) nuclear magnetic resonance (NMR) spectroscopy analysis indicated minimal microbial degradation of cigarette filters (Bonanomi et al. 2015). This stability may be due to the nitrogen limitations in the butts. Studies have been conducted to develop methods to accelerate the degradation of cigarette butts. Honso et al. (2007) found that radical reactions of cellulose acetate by photo-irradiation can lead to oxidation and random cleavage of cellulose acetate. In addition, the authors found benzophenone additives effectively accelerated the degradation of cellulose acetate under ambient environment conditions.

Some states have considered legislation that would require filters to degrade faster (Robertson et al. 2012). One method to meet this requirement is to alter filters so they start degrading once they have been impacted by water. Robertson et al. (2012) showed that hydrolysis can be accelerated by adding weak acids encapsulated in sulfate/phosphate esters. When in contact with water, the encapsulation breaks down and releases weak acids that attack the cellulose acetate.

\subsubsection{Smoking Method}

The demand for data regarding the health impacts of mainstream and sidestream smoke led to the development of complex smoking machines. The operation of these machines is guided by ISO 3308:2012: Routine analytical cigarette-smoking machine - Definitions and standard conditions, details of which are summarized in Section 4.2.4 (ISO3308 2012). The standard gives a single set of operating conditions for smoking machines, while in reality smokers have a wide range of habits that can impact emissions. For instance, Purkis et al. (2010) demonstrated that increased puffing intensity decreased the tar, nicotine and carbon monoxide yields due to more rapid burning of the cigarette. Another study evaluated the tar and nicotine emissions from both human smoking and machine smoking across seventeen brands of cigarettes (St Charles et al. 2010). The comparison of nicotine and tar yields between human smoking and machine smoking was non-linear and scattered. In addition, the relationship was not consistent across various brands. A third study found lower environmental tobacco concentrations generated by smokers compared to smoking machine-generated sidestream smoke (P.R. Nelson 1998). However, the authors did not account for sorption to the smokers' clothes and skin.

Hence, there can be differences in emissions from a cigarette using a smoking machine compared to a human-smoked cigarette. If the mainstream smoke is different, it is likely that the chemical composition of a cigarette butt is also different.

\subsection{Impacts of Cigarette Butts on Human Health and Environment}

Cigarette butts may impact human health and the environment through direct and indirect exposure routes. Only two studies have reported direct human health effects related to the ingestion of cigarette 
products. Novotny et al. (2011) reviewed published and grey literature regarding cigarette butt waste consumption by children, pets and wildlife, and found that accidental ingestion of cigarettes and butts were not uncommon among children, especially those under 6 years of age. Significant toxicity from the ingestion of cigarette products in children has been reported to be rare, with vomiting within 20 minutes being the most common symptom (McGee et al. 1995). The impacts of cigarette butts on cells and animals have been documented in a broader series of studies, as described below.

\subsubsection{In Vitro Assay}

In vitro assays have been used to evaluate the genotoxic potential of cigarette butts. Di Giacomo, et al., (2015) tested the methanol extract from cigarette butts in the bacterial reverse mutation assay (with Salmonella typhimurium TA98 and TA100 and Escherichia coli WP2uvrA strains) and found that it was mutagenic. On the other hand, some compounds can provide protection from the mutagenic effects of cigarette butts. Di Giacomo et al. (2015) found that natural sesquiterpenes (caryophyllene and caryophyllene oxide) can be used to inhibit the mutagenicity of cigarette butts. Such compounds may have a place in remedial strategies to decontaminate cigarette butt waste.

\subsubsection{Animal Exposure}

A variety of detrimental effects have been observed in animals exposed to cigarette butts directly or indirectly via leachate in water. However, no studies were found on the effects of cigarette butts in animals via airborne exposure routes. Animals reported to be affected by cigarette butts include birds, mosquitoes, snails, ragworms and fish. Birds use cellulose from smoked cigarette butts as lining material in nests, which helps to reduce ectoparasites in the nests, possibly because nicotine repels anthropods (Suarez-Rodriguez et al. 2013, Waters 2013). However, signs of genotoxicity in house finches have been seen to increase with the amount of butt cellulose in their nests (Suarez-Rodriguez and Garcia 2014). In addition, mosquitoes that have hatched in water environments containing cigarette butts have been shown to have shorter life spans (Dieng et al. 2014) and higher mortality rates during developmental stages (Dieng et al. 2013). Cigarette butt leachate in seawater has also been shown to cause mortality and behavioral modifications in snails (Booth et al. 2015). Ragworms experienced significant weight loss and DNA damage when exposed to levels of filtration that were over sixty times less concentrated compared to average runoff in urban settings (Wright et al. 2015).

Slaughter et al. (2011) investigated the LC50 (concentration in water at which $50 \%$ of test subject will die) of cigarette butt leachate in fish. Their results indicated that the toxicity of cigarette butt leachate increases from unsmoked cigarette filters (no tobacco) to smoked cigarette filters (no tobacco) to smoked cigarette butts (smoked filter + tobacco). Smoked cigarette butts had an LC50 of about one cigarette butt $\mathrm{L}^{-1}$ in both the marine topsmelt (Atherinops affinis) and the freshwater fathead minnow (Pimephales promelas).

\subsection{Alternative Uses for Cigarette Butts}

Cigarette butts are frequently randomly discarded in the urban environment. However, there can be productive uses for cigarette butts. For example, they have been used as a media for biofilm growth in anaerobic digestors (Sabzali et al. 2012). Cigarette filters have also been used to store energy by heat treating them under a nitrogen atmosphere to prepare $\mathrm{N}$-doped mesoporous / microporous carbon material (Minzae et al. 2014). This material provides a favorable pathway for electrolyte permeation and contact probability. The latent energy in cigarette filter waste has also been harnessed to supply heat recovery units in industry (Nagarajan 2014). 
Another application of used cigarette filters is as a source of porous carbon material for producing activated carbon (Masoudi Soltani and Kazemi Yazdi 2012, Salman et al. 2014, Soltani et al. 2015). Soltani et al. (2015) showed that the charred carbon from cigarette filters can remove lead from water. Chen et al. (2015) synthesized mesoporous carbonaceous materials on the surface of cigarette filters, and these materials exhibited enhanced performance in phenol adsorption and carbon dioxide capture. 


\section{Experimental Plan for Phase II}

The above literature review on emissions from cigarette butts shows that airborne emissions from cigarette butts is not well understood and needs further study. The purpose of Section 4 is to describe an experimental plan intended to quantify the emissions of a selected number of chemicals emitted from cigarette butts into air under a variety of environmental conditions. The design of the experimental plan focuses on three potential exposure scenarios for discarded cigarette butts:

- Outdoor Exposure. In this scenario, discarded cigarette butts in a park or playground may impact children or adults. Furthermore, emissions from discarded cigarette butts in a trash or cigarette receptacle may impact people or even be drawn into a building if it is located near an outdoor air intake or other building opening.

- Indoor Exposure. In this scenario, discarded cigarettes butts located in an ashtray in a building may impact occupants.

- Car Exposure. In this scenario, occupants may be present in hot cars with discarded cigarettes butts despite not being present during the smoking event.

The experimental plan aims to be a screening analysis that can help address these potential exposure scenarios.

\subsection{Screening Tool: Headspace Analysis}

As discussed in the literature review, air emissions from cigarette butts can be a complex mixture of thousands of chemicals. These chemicals will interact with the environment, whether it be the photooxidation of the volatile chemicals or sorption to surfaces and particles of less volatile chemicals. Given there is a lack of data on what chemicals will emit from cigarette butts, it is proposed to first develop a screening tool to first determine which chemicals are important and estimate how long these chemicals are present. The screening tool to be used for this task is automated headspace analysis.

Once a cigarette butt is placed in a headspace vial, chemicals will be emitted from the butt under the given test conditions (temperature, flow rate and desorption time). While the emission rate of the chemical in the vial will not be the same emission rate of the cigarette butt in the exposed environment, the mass released during headspace analysis will allow comparison of the emission from a cigarette butt expose to different conditions. For instance, the headspace analysis would show different masses released for cigarettes stored outdoors for three months during summer and in an indoor controlled environment for three months. Further details of the headspace analysis are described in Section 4.2.1. If resources permit, the emittable mass over time generated from headspace analysis (Figure 2 ) will be compared with an emission experiment conducted in a large chamber. 


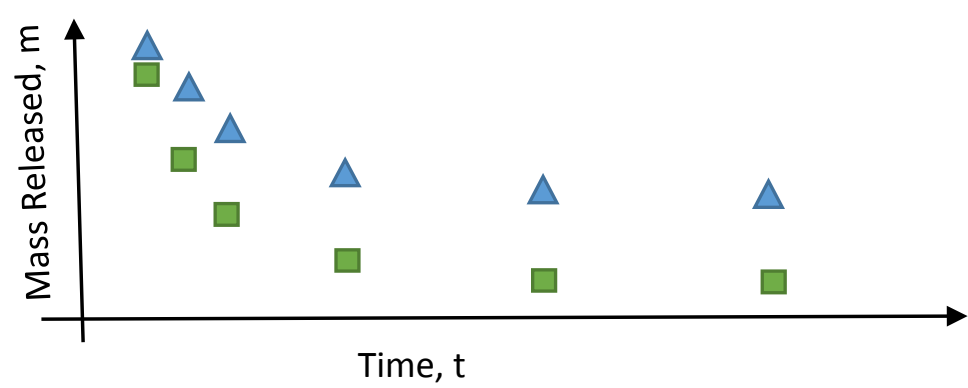

Figure 2: Illustrative data from headspace analysis of a chemical in two different experiments (e.g. squares from cigarettes in an outdoor environment versus triangles from cigarettes in an indoor environment). Each symbol represents the mass of the chemical derived from headspace analysis for a cigarette butt exposed under different, controlled environmental conditions over time.

This screening tool will be used in the tasks described below to allow comparison of how cigarette butts age under different conditions for Tasks 2 through Tasks 5.

- Task 1: Method development

- Task 2: Initial experiments

- Task 3: SPHERE experiments

- Task 4: Large chamber experiments

- Task 5: Roof top experiments

The following sections describe the proposed activities for each of the tasks.

\subsection{Task 1: Method Development}

There are a range of parameters that need to be determined prior to conducting the experiments in Tasks 2 through 5 . The parameters include but are not limited to the following:

- Parameters for headspace analysis (temperature, flow, sample volume, split, etc.)

- Cigarette brand

- Length of cigarette butts

- Method for generation of cigarette butts

- Compounds to be quantified

- Method validation

These parameters will be determined based on experimental results and discussion with FDA. NIST First Level Hazard Reviews and standard practices for all NIST laboratory work will also be developed as part of this task.

\subsubsection{Headspace Analysis}

Headspace analysis will be used to determine the emittable mass of each tested cigarette butt. After exposing a cigarette but to under a specific set of environmental conditions for a defined time, the cigarette butt will be placed in a sealed headspace vial and incubated at a defined temperature (above room temperature) for a defined time (Figure 3A). A constant volume of air from the headspace of the vial will then be collected using conditioned Tenax-TA tubes (Figure 3B). The sample will be thermally desorbed (TD) and quantified using GC-MS under defined conditions. To increase the sensitivity and reduce the analysis time of the method, experiments 
will be conducted to optimize the parameters, including the incubation temperature, incubation time, sampling volume, and TD-GC-MS conditions.
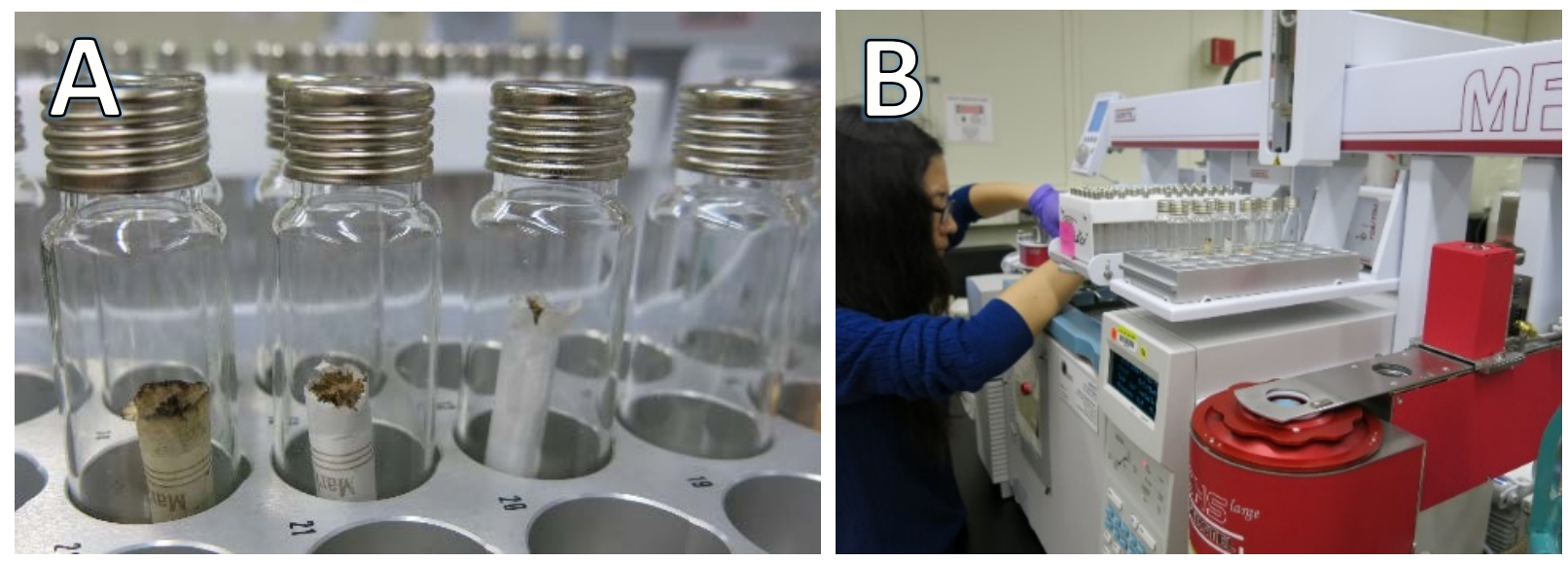

Figure 3: A) Incubation of cigarette butts in headspace vials. B) Sampling from headspace vials.

\subsubsection{Selection of Cigarette Brand}

Unless otherwise noted, three of the most popular cigarette brands based on FDA information will be selected for preliminary study during the method development period. One of these three brands will be selected for all other experiments based on its performance in the headspace analysis.

\subsubsection{Determination of Butt Length}

Roughly 50 cigarette butts will be randomly collected from a variety of public places. These cigarette butt lengths will be measured. The median value of the measured lengths will be used as the butt length for all the experiments.

\subsubsection{Generation of Cigarette Butt}

To make data comparable across different experiments, the generation of cigarette butts must be consistent. Hence, a smoking machine will be employed, as described below, to generate most of the cigarette butts. To determine how representative smoking machine butts are, they will be compared with butts generated by human smoking. Cigarette butts will be collected from NIST outdoor ashtrays shortly after humans complete smoking. Smokers will not be asked to smoke. Rather signs will be placed at designated smoke areas asking smokers to place cigarette butts in provided headspace vials. The signs will also ask the smokers to record the number puffs and brand on the vial. This collection method has preliminary approval from the by the NIST Institutional Review Board (IRB) and will be fully reviewed prior to conducting the research. Freshly generated cigarette butts of the same brand and similar lengths from both the smoking machine and human smoking will be measured using the headspace analysis. Both the qualitative and quantitative differences between emissions of cigarette butts by the smoking machine and human smoking will be examined to determine the representativeness of the smoking machine. Adjustments for the butts generated from the smoking machine will be made if necessary.

A smoking machine will be constructed to partially fulfill the requirements described in ISO 3308 . This device will draw a fixed volume of air through the cigarette (a puff) in a sequence similar to the puff profile defined in ISO 3308 (2012). As per ISO 3308:2012, the device will hold $9.0 \mathrm{~mm}$ of the butt end of the cigarette using labyrinth seals in a restricted smoking fashion (filter ventilation 
holes covered). The device will be placed in a NIST fume hood configured to allow air speeds of $170 \mathrm{~m} / \mathrm{s}$ to $230 \mathrm{~m} / \mathrm{s}$, temperatures of $22{ }^{\circ} \mathrm{C} \pm 2{ }^{\circ} \mathrm{C}$, and relative humidity of $60 \% \pm 5 \%$.

The device will deviate from ISO 3308:2012 in two important ways: 1) a smoke trap will not be included in the machine, since measurement of the mainstream smoke is outside the scope of this research; and 2) the machine may not produce a bell-shaped puff profile due to technical challenges. This will likely not influence the experiments in this study as long as the puff profile is consistent for different cigarette butts.

\subsubsection{Target Compound List}

The above literature review shows that certain compounds with high boiling points in cigarette smoke, such as metals and TSNAs, mainly exist in the particle phase in mainstream and sidestream smoke due to their low volatility. Since, it would be difficult to detect these chemicals in the air emissions from cigarette butts, we are not selecting them as target compounds for quantification in this investigation. Compounds which have moderate boiling points and weak to moderate sorption will be the main target compounds; these include nicotine, and PAHs. Both compounds are found on the United States Food and Drug Administrations' Harmful and Potentially Harmful Constituents in Tobacco Products and Tobacco Smoke: Established List. It is unclear whether other compounds such as hydrogen cyanide will be able to be detected with the proposed experimental techniques.

Preliminary headspace analyses were conducted using two cigarette butts (one freshly smoked and one found on the ground). The results showed that cigarette butts can emit a large number of chemicals, with over 50 different peaks identified from the chromatograms shown in Figure 4.

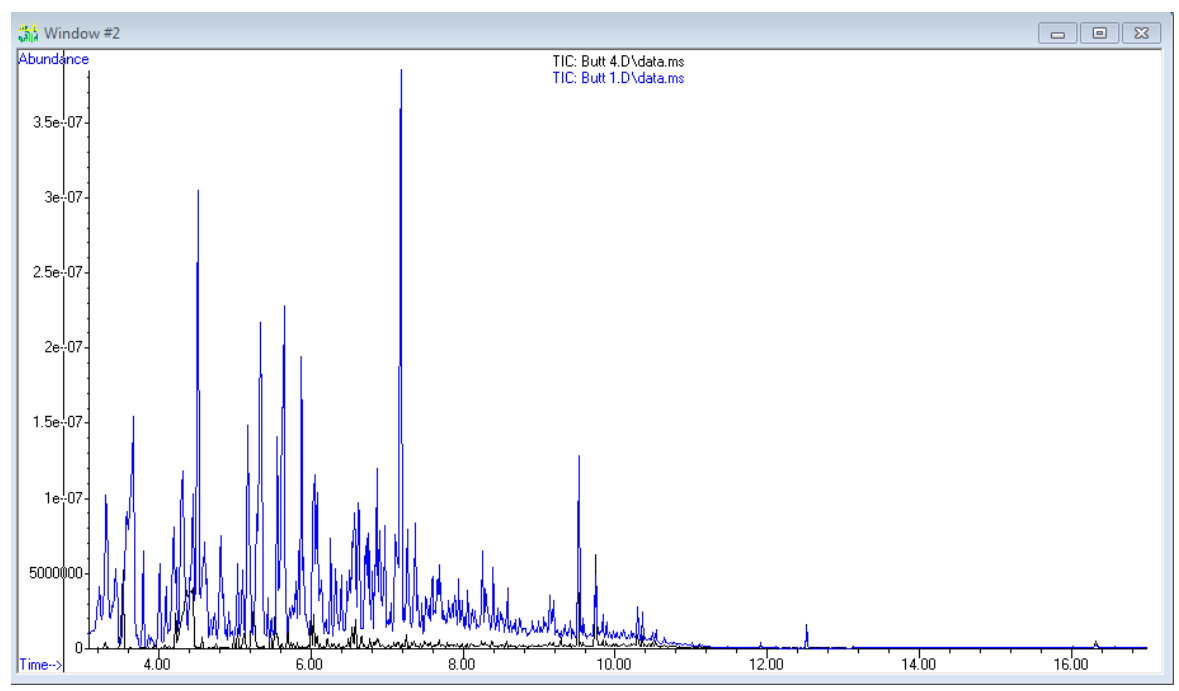

Figure 4: Illustrative data from headspace analysis for two different cigarette butts. The blue trace was from a freshly smoked cigarette; the black trace was from a cigarette found on the ground. The $x$-axis represents time in minutes, while the $y$-axis represents chemical abundance in dimensionless units.

For the freshly generated cigarette butt, the peaks with top five largest areas were identified as dlimonene, toluene, pyridine, benzene and styrene. The top five peaks for the cigarette butt found on the ground were nicotine, 3-methyl-pyridine, furfural, pyridine, and methyl-pyrazine, which is very different from the chemicals emitted from the freshly generated cigarette butt. This indicates 
that the target chemicals to be quantified need to be carefully chosen to ensure that the data captures both short- and long-term emissions.

For proposed experiments, the compounds will be first tentatively identified with the NIST mass spectral library and chosen as target compounds for qualitative analysis when the quality of the match is higher than $90 \%$. Then, the top ten identified chemicals based on response area, and being both carcinogenic and with good peak shapes, will be chosen as target compounds for quantitative analysis.

\subsubsection{Method Validation}

Once method parameters and target chemicals are determined method validation will be conducted. TD-GC-MS parameters for the quantitative target compounds will be determined to ensure the linear correlation coefficient of the corresponding standard curve is higher than 0.98 . The recovery of quantified chemicals in the headspace vials will be determined. In addition, method detection limit and repeatability experiments will be conducted. Reported data will include combined uncertainties.

\subsection{Task 2: Initial Experiments}

As described in Section 3.4, there are a wide range of factors that can influence the emissions from a cigarette butt. The cigarette butt length and brand (which will dictate the filter type) will be defined during Task 1: Method Development. Task 2: Initial Experiments will examine the influence of some of the remaining factors described in Section 3.4, including: 1) smoking condition (puffing parameters); 2) temperature; and 3) moisture (to account for rain).

\subsubsection{Smoking Condition}

Freshly generated cigarette butts from a smoking machine with different puffing parameters (number and airflow) will be examined using headspace analysis. All butts will be analyzed after they have reached room temperature.

\subsubsection{Temperature}

A micro-chamber system, as shown in Figure 5, will be used for studying the cigarette butt emissions after aging at different temperatures. The butts will be placed in the micro-chambers for up to a week at different temperatures with constant flows. Then, the conditioned cigarette butts will be put into the headspace vial for analysis. The exact number of experiments will be determined based on technical complexity. 

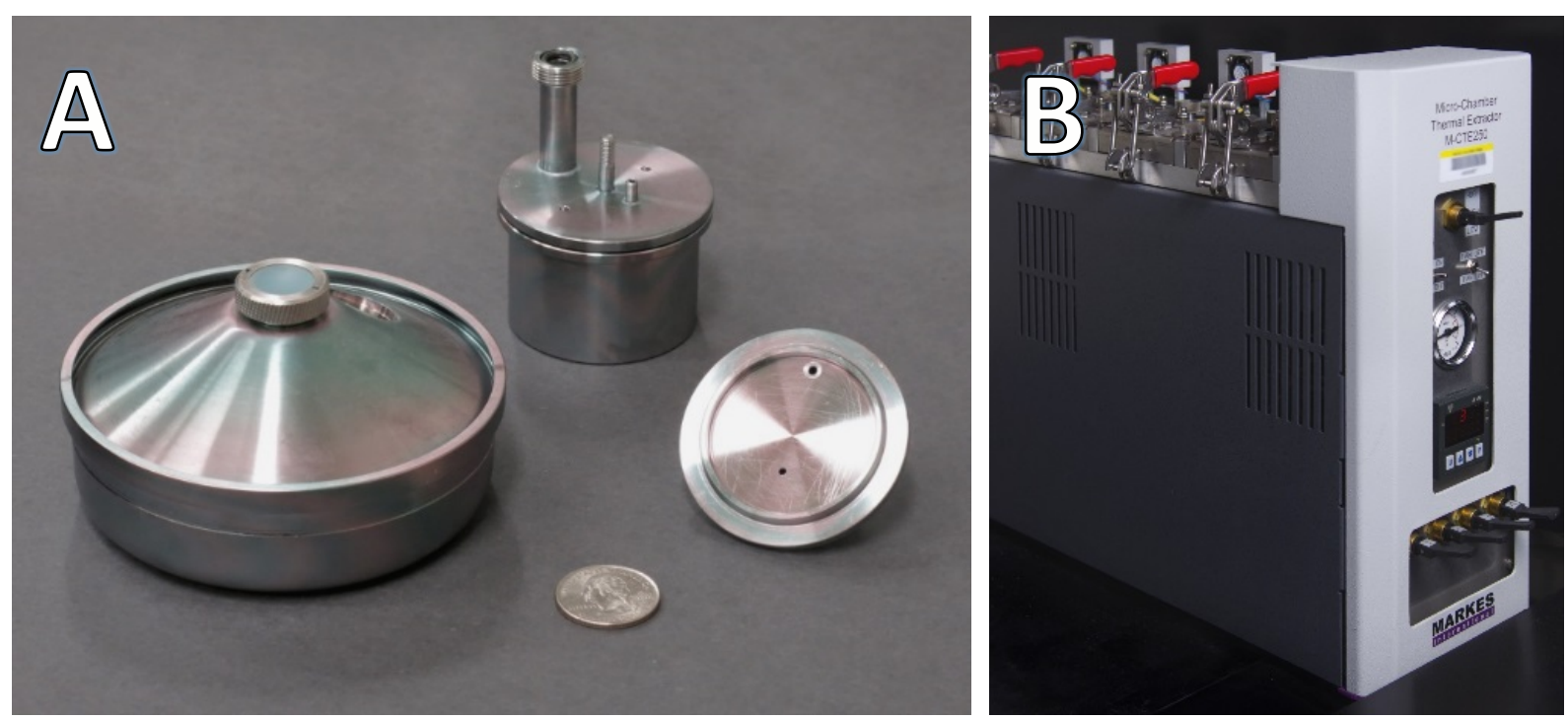

Figure 5: A) Different kinds of micro-chambers. B) Markes micro-chamber system

\subsubsection{Moisture}

Cigarette butts will be saturated with water to simulate a rain event. Added moisture to the cigarettes will be measured by mass change. After wetting, the butts will be placed in headspace vials for analysis. A subset of the wet butts will be placed in a micro-chamber system for up to a week to simulate a drying event prior to the headspace analysis. The exact number of experiments will be determined based on technical complexity.

\subsection{Task 3: Large Chamber Experiments}

To mimic indoor conditions, cigarette butts will be placed in a large stainless steel walk-in chamber, shown in Error! Reference source not found., for a defined time. The chamber will be operated at typical indoor test conditions $\left(20^{\circ} \mathrm{C}\right.$, air change rate of $\left.1 \mathrm{~h}^{-1}\right)$. After placement in the chamber, butts will be removed at various points in time for headspace analysis. The exact number of butts and length of aging will be determined based on technical complexity and experimental design. The emissions from the cigarette butts in the large chamber will be compared to emissions from butts in the ultra violet radiation exposure (Section 4.5) and rooftop experiments (Section 4.6) to determine the impact of exposure to radiation and outdoor conditions on emissions, respectively. 

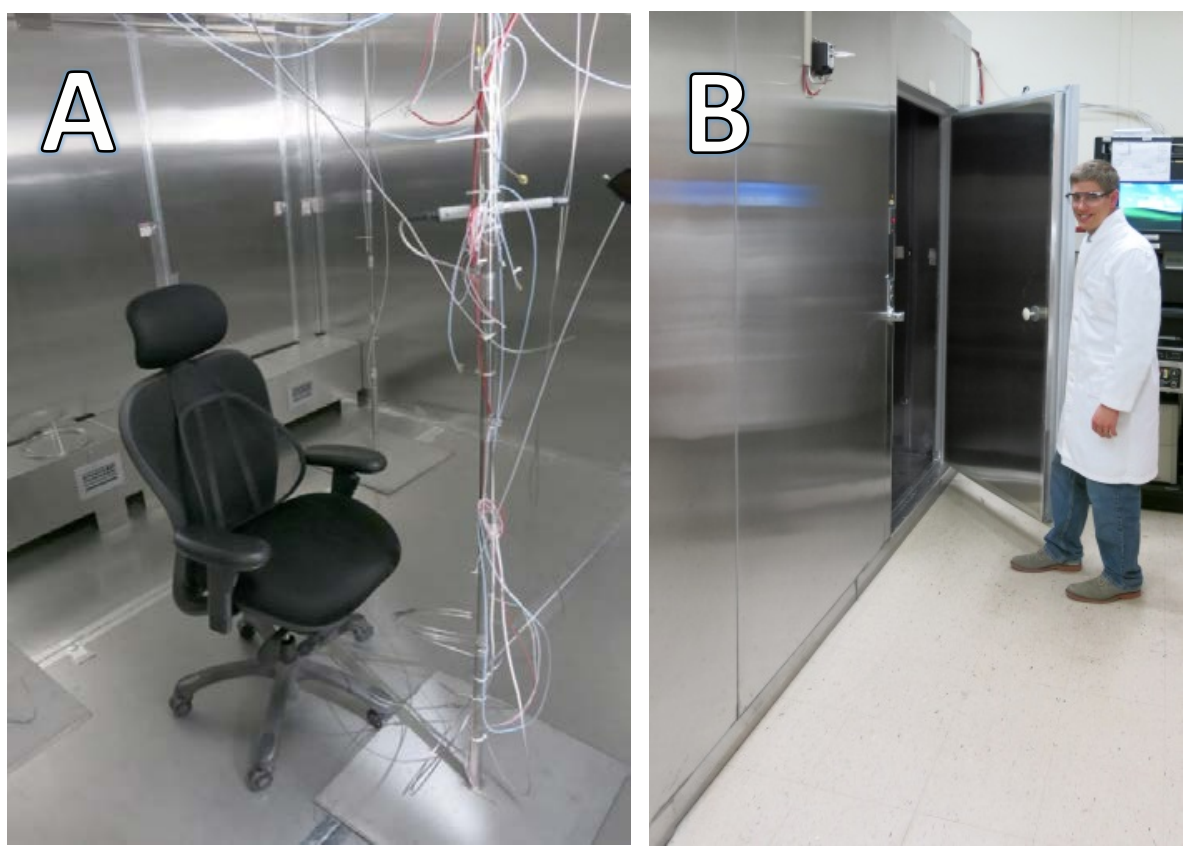

Figure 6: A) Inside of the large chamber. B) Outside of the large chamber

If there is enough time, a chamber emission study will be conducted using the large chamber. In these experiments, a large number of cigarette butts will be placed in the large chamber. The air will be sampled at regular intervals using a Tenax TA sorbent tube. At some point in time, a $100 \mathrm{~cm}^{2}$ section of the wall will be wiped with a methanol impregnated pad. The pad will be extracted to determine the sorption rate of the chemicals to the chamber walls, which will be used to determine the average emission rate in the chamber. The chamber will be ventilated with outdoor air, which will contain particles. Particle concentrations in the chambers will be measured to allow for estimation of the chemical concentrations on particles using established methods (Little et al. 2012Little et al. 2012). Once the average cigarette butt emission rate in the chamber is known, it will be compared to the screening headspace analysis to demonstrate the relevance of the screening data (Error! Reference source not found.).

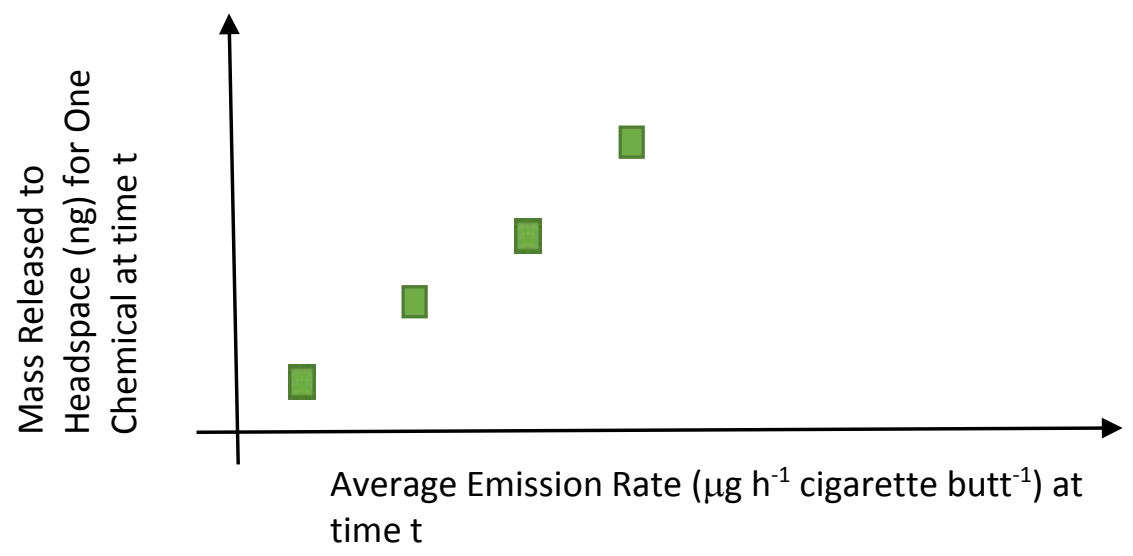

Figure 7: Illustrative data comparing mass recovered during headspace analysis to average emission rate at different times in a large chamber). 


\subsection{Task 4: SPHERE Experiments}

Another factor that possibly can influence the emissions from the cigarette butts is radiation exposure. The NIST Simulation Photo-degradation via High Energy Radiation Emission (SPHERE) chambers, as shown in Figure 8, will be used to study cigarette butt emissions under different UV radiation conditions. Fresh cigarette butts from a smoking machine will be placed in the SPHERE chamber with different UV radiation intensities. After placement in the chamber for different aging durations, cigarette butts will be put in headspace vials for analysis.
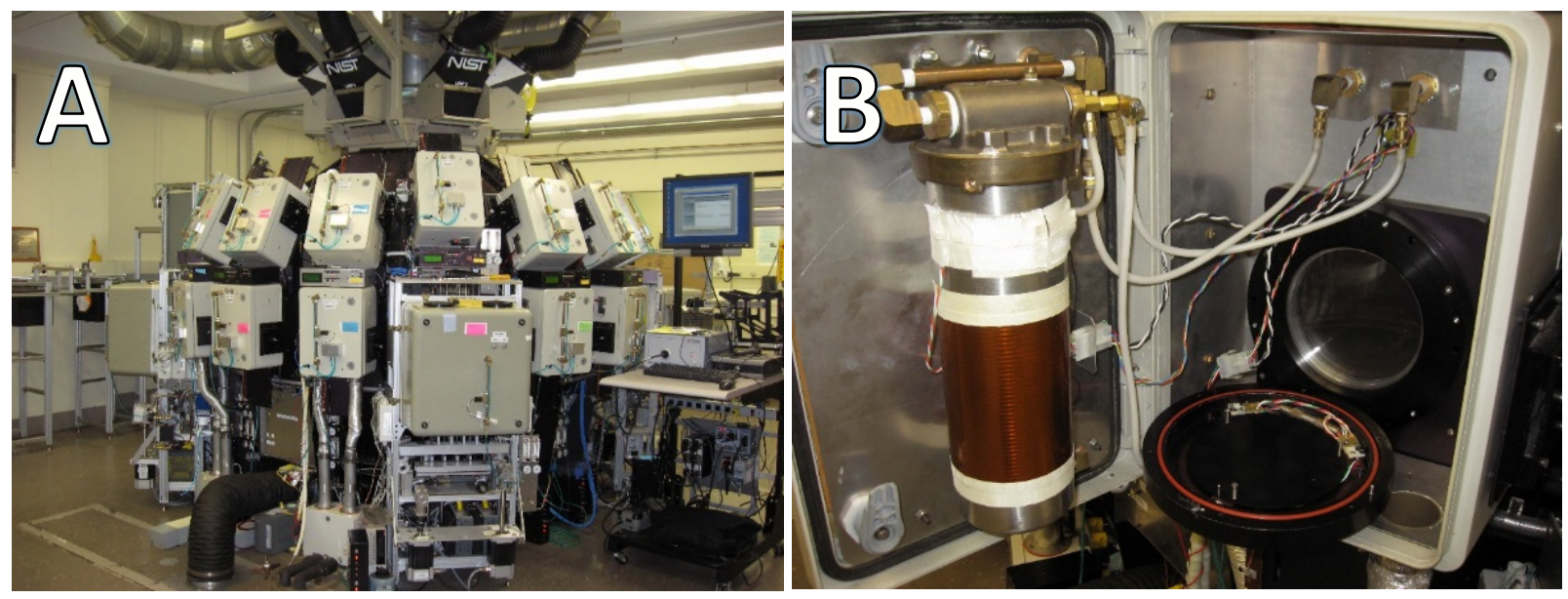

Figure 8: A) Simulation Photo-degradation via High Energy Radiation Emission (SPHERE) system.

B) An individual SPHERE chamber.

\subsection{Task 5: Roof Top Experiments}

To understand cigarette butt emissions in the outdoor environment, this task will compare emissions from cigarette butts that have been placed on a rooftop during four different seasons. Solar irradiance, UV radiation, cloud cover, temperature, wind speed, pressure, relative humidity, rain and snow accumulations will all be recorded while the butts are exposed on the roof to determine if any of these parameters has a significant impact on the emissions. After placement on the roof for different time periods, butts will be removed for headspace analysis. Some butts may be placed on the roof under cover that limits direct sunlight and rain but maintains butts at the same temperature, humidity and wind conditions. The exact number of butts and length of aging will be determined based on technical complexity and experimental design.

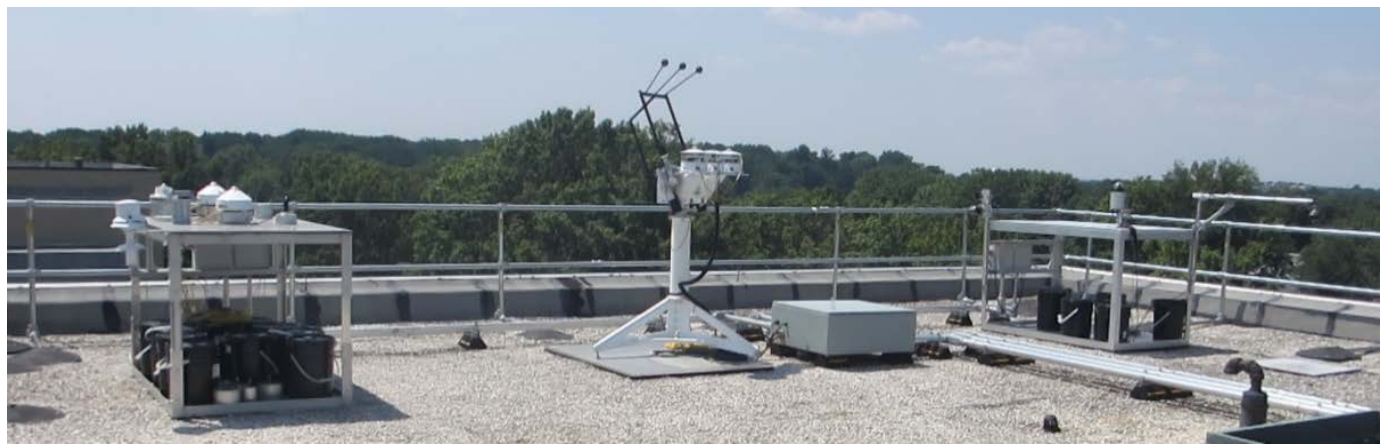

Figure 9: NIST solar observation equipment. Cigarette butts aged for Task 7 would be located near this infrastructure. 


\subsection{Schedule}

The experimental tasks are scheduled to take two years to complete. The experimental schedule will commence at the conclusion of Phase 1 of this project, which corresponds to final delivery of this document to FDA.

\section{Table 4: Proposed Experimental schedule}

\begin{tabular}{|llll|}
\hline Task & Purpose & Duration & $\begin{array}{l}\text { Headspace } \\
\text { Analysis }\end{array}$ \\
\hline $\begin{array}{l}\text { Method } \\
\text { Development }\end{array}$ & $\begin{array}{l}\text { Determine brand and length of } \\
\text { cigarette butt, method to generate } \\
\text { cigarette butt, and parameters for } \\
\text { headspace analysis }\end{array}$ & N months \\
\hline $\begin{array}{l}\text { Initial } \\
\text { experiments }\end{array}$ & $\begin{array}{l}\text { Examine the influence of factors on } \\
\text { emissions, including smoking } \\
\text { condition, temperature, and } \\
\text { moisture }\end{array}$ & Weekly \\
\hline $\begin{array}{l}\text { SPHERE } \\
\text { experiments }\end{array}$ & $\begin{array}{l}\text { Examine the influence of UV } \\
\text { radiation on emissions }\end{array}$ & 12 months* & Monthly \\
\hline $\begin{array}{l}\text { Large Chamber } \\
\text { Experiments }\end{array}$ & $\begin{array}{l}\text { Study the emissions in a large } \\
\text { chamber environment }\end{array}$ & 6 months* & Monthly \\
\hline $\begin{array}{l}\text { Roof Top } \\
\text { Experiments }\end{array}$ & $\begin{array}{l}\text { Study the emissions in the outdoor } \\
\text { environment }\end{array}$ & 6 months* & Monthly \\
\hline $\begin{array}{l}\text { Data Evaluation/ } \\
\text { Report }\end{array}$ & $\begin{array}{l}\text { Document all project tasks } \\
\text { S }\end{array}$ & 3 months & N/A \\
\hline
\end{tabular}

* concurrently

\section{Summary}

Cigarette butts can contain many of the same chemicals found in mainstream and sidestream smoke, making them a potential source of chemical exposure in both indoor and outdoor environments. Many studies have analyzed chemicals in cigarette butts and quantified chemicals emitted from cigarette butts into water. However, there are limited data on emissions from cigarette butts into air. The emission rates from cigarette butts to the air may be minimal for some heavy chemicals (e.g., metals, TSNAs), but could be high for more volatile chemicals (e.g., nicotine, pyridine, benzene). In addition, the airborne emissions of cigarette butts can be influenced by the cigarette brand, filter material, butt length, environmental temperature, airflow around the cigarette, number of puffs during smoking, degradation of the butt, and smoking method. Based on the results of the literature review presented in this report, more data are needed on the airborne emission rates of cigarette butts. The proposed research aims to fill this data gap by using a screening tool (e.g. headspace analysis) to examine the airborne emission of chemicals from cigarette butts under various environmental conditions (small chamber, large chamber, outdoor, and chamber with enhanced ultra violet radiation). 


\section{Disclaimer}

Certain trade names or company products are mentioned in the text to adequately specify the experimental procedure and equipment used. In no case does such identification imply recommendation or endorsement by the National Institute of Standards and Technology, nor does it imply that the equipment is the best available for the purpose.

\section{Acknowledgements}

This work was funded under U.S. Food and Drug Administration Interagency Agreement \#244-15-9012.

\section{References}

Ashley, D. L., R. J. O'Connor, J. T. Bernert, C. H. Watson, G. M. Polzin, R. B. Jain, D. Hammond, D. K. Hatsukami, G. A. Giovino, K. M. Cummings, A. McNeill, L. Shahab, B. King, G. T. Fong, L. Q. Zhang, Y. Xia, X. Z. Yan and J. M. McCraw (2010). Effect of differing levels of tobacco-specific nitrosamines in cigarette smoke on the levels of biomarkers in smokers. Cancer Epidemiology Biomarkers \& Prevention 19(6): 1389-1398.

Awji, E. G., H. Chand, S. Bruse, K. R. Smith, J. K. Colby, Y. Mebratu, B. D. Levy and Y. Tesfaigzi (2015). Wood smoke enhances cigarette smoke-induced inflammation by inducing the aryl hydrocarbon receptor repressor in airway epithelial cells. American Journal of Respiratory Cell and Molecular Biology 52(3): 377-386.

Baker, R. R. (1987). A review of pyrolysis studies to unravel reaction steps in burning tobacco. Journal of Analytical and Applied Pyrolysis 11: 555-573.

Becherucci, M. E. and J. P. S. Pon (2014). What is left behind when the lights go off? Comparing the abundance and composition of litter in urban areas with different intensity of nightlife use in mar del plata, argentina. Waste Management 34(8): 1351-1355.

Boldridge, D. W. and B. J. Ingebrethsen (1986). Coagulation and filtration of mainstream cigarette smoke in the tobacco rod of a cigarette. Aerosols: Formation and Reactivity, Proceedings - Second International Aerosol Conference: 459-461.

Bonanomi, G., G. Incerti, G. Cesarano, S. A. Gaglione and V. Lanzotti (2015). Cigarette butt decomposition and associated chemical changes assessed by c-13 cpmas nmr. Plos One 10(1).

Booth, D. J., P. Gribben and K. Parkinson (2015). Impact of cigarette butt leachate on tidepool snails. Marine Pollution Bulletin 95(1): 362-364.

Brinkman, M. C., J. C. Chuang, S. M. Gordon, H. Kim, R. R. Kroeger, G. M. Polzin and P. A. Richter (2012). Exposure to and deposition of fine and ultrafine particles in smokers of menthol and nonmenthol cigarettes. Inhalation Toxicology 24(5): 255-269.

Brunnemann, K. D., E. J. Mitacek, Y. Liu, T. Limsila and M. Suttajit (1996). Assessment of major carcinogenic tobacco-specific n-nitrosamines in thai cigarettes. Cancer Detection and Prevention 20(2): 114-121.

Carolina Talio, M., M. Alesso, M. Acosta, R. Olsina and L. P. Fernandez (2013). Determination of cadmium in tobacco by solid surface fluorescence using nylon membranes coated with carbon nanotubes. Talanta 107: 61-66.

Chapman, S. (2006). Butt clean up campaigns: Wolves in sheep's clothing? Tobacco Control 15(4). 
Chen, A., Y. Li, Y. Yu, Y. Li, L. Zhang, H. Lv and L. Liu (2015). Mesoporous carbonaceous materials prepared from used cigarette filters for efficient phenol adsorption and co2capture. Rsc Advances 5(130): 107 299-107 306.

Chen, B., W. D. Wang and Y. M. Huang (2012). Cigarette filters as adsorbents of solid-phase extraction for determination of fluoroquinolone antibiotics in environmental water samples coupled with high-performance liquid chromatography. Talanta 88: 237-243.

Chen, S., Y. He, G. Shen, X. Sun, C. Nie, X. Zhang, Y. Lai, X. Li and H. Liu (2015). Application of materials for reducing volatile carbonyl compounds in cigarette filter. Acta Tabacaria Sinica 21(1): 6-12.

Claereboudt, M. R. (2004). Shore litter along sandy beaches of the gulf of oman. Marine Pollution Bulletin 49(9-10): 770-777.

Clark, T., E. Kaussmann, E. Romer and G. Schepers (1998). The fate of imidacloprid in tobacco smoke of cigarettes made from imidacloprid-treated tobacco. Pesticide Science 52(2): 119-125.

Clayton, P. M., A. Cunningham and J. D. H. van Heemst (2010). Quantification of four tobacco-specific nitrosamines in cigarette filter tips using liquid chromatography-tandem mass spectrometry. Analytical Methods 2(8): 1085-1094.

Coggins, C. R. E., W. J. McKinney and M. J. Oldham (2013). A comprehensive evaluation of the toxicology of experimental, non-filtered cigarettes manufactured with different circumferences. Inhalation Toxicology 25: 69-72.

Daher, N., R. Saleh, E. Jaroudi, H. Sheheitli, T. Badr, E. Sepetdjian, M. Al Rashidi, N. Saliba and A. Shihadeh (2010). Comparison of carcinogen, carbon monoxide, and ultrafine particle emissions from narghile waterpipe and cigarette smoking: Sidestream smoke measurements and assessment of second-hand smoke emission factors. Atmos Environ (1994) 44(1): 8-14.

Dalluge, J., L. L. P. Van Stee, X. Xu, J. Williams, J. Beens, R. J. J. Vreuls and U. A. T. Brinkman (2002). Unravelling the composition of very complex samples by comprehensive gas chromatography coupled to time-of-flight mass spectrometry: Cigarette smoke. Journal of Chromatography A 974(12): 169-184.

Demirci, A. (2014). Factorial design optimization of solid-phase microextraction for high-performance liquid chromatography-ultraviolet spectrometry analysis of polycyclic aromatic hydrocarbons in cigarette filter tar. Polycyclic Aromatic Compounds 34(2): 115-134.

Demirci, A. and E. Alver (2013). Determination of polycyclic aromatic hydrocarbons in cigarette filter tar by means of hollow-fiber liquid phase microextraction-hplc-uv system. Journal of Liquid Chromatography \& Related Technologies 36(5): 628-647.

Demirci, A. and M. Ekiz (2013). Statistical classification of cigarettes using high pressure liquid chromatograms of cigarette filter tar as fingerprint. Asian Journal of Chemistry 25(17): 9860-9864.

Di Giacomo, S., G. Mazzanti and A. Di Sotto (2015). Mutagenicity of cigarette butt waste in the bacterial reverse mutation assay: The protective effects of -caryophyllene and -caryophyllene oxide. Environmental Toxicology and Chemistry.

Dieng, H., S. Rajasaygar, A. H. Ahmad, H. Ahmad, C. S. M. Rawi, W. F. Zuharah, T. Satho, F. Miake, Y. Fukumitsu, A. R. Saad, I. Abd Ghani, R. E. M. Vargas, A. H. Ab Majid and S. AbuBakar (2013). Turning cigarette butt waste into an alternative control tool against an insecticide-resistant mosquito vector. Acta Tropica 128(3): 584-590. 
Dieng, H., S. Rajasaygar, A. H. Ahmad, C. S. M. Rawi, H. Ahmad, T. Satho, F. Miake, W. F. Zuharah, Y. Fukumitsu, A. R. Saad, S. A. Hamid, R. E. M. Vargas, A. H. Ab Majid, N. Fadzly, N. F. Abu Kassim, N. A. Hashim, I. A. Ghani, F. B. Abange and S. AbuBakar (2014). Indirect effects of cigarette butt waste on the dengue vector aedes aegypti (diptera: Culicidae). Acta Tropica 130: 123-130.

Ding, Y. S., J. Ward, D. Hammond and C. H. Watson (2014). Mouth-level intake of benzo a pyrene from reduced nicotine cigarettes. International Journal of Environmental Research and Public Health 11(11): 11898-11914.

Ding, Y. S., L. Q. Zhang, R. B. Jain, N. Jain, R. Y. Wang, D. L. Ashley and C. H. Watson (2008). Levels of tobacco-specific nitrosamines and polycyclic aromatic hydrocarbons in mainstream smoke from different tobacco varieties. Cancer Epidemiology Biomarkers \& Prevention 17(12): 3366-3371.

Dittrich, D. J., R. T. Fieblekorn, M. J. Bevan, D. Rushforth, J. J. Murphy, M. Ashley, K. G. McAdam, C. Liu and C. J. Proctor (2014). Approaches for the design of reduced toxicant emission cigarettes. Springerplus 3.

Djordjevic, M. V., C. W. Sigountos, D. Hoffmann, K. D. Brunnemann, M. R. Kagan, L. P. Bush, R. D. Safaev, G. A. Belitsky and D. Zaridze (1991). Assessment of major carcinogens and alkaloids in the tobacco and mainstream smoke of ussr cigarettes. International Journal of Cancer 47(3): 348-351.

Duan, L., Y. Gu, Y. Zhang, P. Sheng, X. Yan, X. Xu and C. Wang (2014). Effects of length and position of grooved section in grooved acetate filter on yield of cigarette smoke. Tobacco Science and Technology(11): 5-9.

Eatough, D. J., L. D. Hansen, E. A. Lewis (1990). The chemical characterization of environmental tobacco smoke. Environmental Technology.

Endo, O., M. Koyano, S. Mineki, S. Goto, K. Tanabe, H. Yajima, T. Ishii and H. Matsushita (2000). Estimation of indoor air pah concentration increases by cigarette, incense-stick, and mosquitorepellent-incense smoke. Polycyclic Aromatic Compounds 21(1-4): 261-272.

Formella, K., T. Braumann and H. Elmenhorst (1992). The influence of different filter parameters on the semivolatile composition of mainstream smoke. Beitrage Zur Tabakforschung International 15(3): 123-128.

Fu, M., J. M. Martinez-Sanchez, I. Galan, M. Perez-Rios, X. Sureda, M. J. Lopez, A. Schiaffino, A. Moncada, A. Montes, M. Nebot and E. Fernandez (2013). Variability in the correlation between nicotine and pm2.5 as airborne markers of second-hand smoke exposure. Environ Res 127: 49-55.

Fukuhara, K., T. Sakaki, H. Sakuma and S. Sugawara (1985). Odor analysis of cigarette butts by a headspace technique. Agricultural and Biological Chemistry 49(7): 2177-2179.

Gaddamidi, V., W. T. Zimmerman, M. Ponte and L. Ruzo (2011). Pyrolysis of c-14 -chlorantraniliprole in tobacco. Journal of Agricultural and Food Chemistry 59(17): 9424-9432.

Galazyn-Sidorczuk, M., M. M. Brzoska and J. Moniuszko-Jakoniuk (2008). Estimation of polish cigarettes contamination with cadmium and lead, and exposure to these metals via smoking. Environmental Monitoring and Assessment 137(1-3): 481-493.

Green, A. L. R., A. Putschew and T. Nehls (2014). Littered cigarette butts as a source of nicotine in urban waters. Journal of Hydrology 519: 3466-3474.

Guo, J., P. Shang, J. Cai, L. Zhao, H. Liu and H. Wang (2015). Determination of hydrogen cyanide retention in cigarette filters with continuous flow analysis. Acta Tabacaria Sinica 21(3): 1-4. 
Haorah, J., L. Zhou, X. J. Wang, G. P. Xu and S. S. Mirvish (2001). Determination of total n-nitroso compounds and their precursors in frankfurters, fresh meat, dried salted fish, sauces, tobacco, and tobacco smoke particulates. Journal of Agricultural and Food Chemistry 49(12): 6068-6078.

Healton, C. G., K. M. Cummings, R. J. O'Connor and T. E. Novotny (2011). Butt really? The environmental impact of cigarettes. Tobacco Control 20: I1-I1.

Hertz-Schunemann, R., S. Ehlert, T. Streibel, C. Liu, K. McAdam, R. R. Baker and R. Zimmermann (2015). High-resolution time and spatial imaging of tobacco and its pyrolysis products during a cigarette puff by microprobe sampling photoionisation mass spectrometry. Analytical and Bioanalytical Chemistry 407(8): 2293-2299.

Hertz, R., T. Streibel, C. Liu, K. McAdam and R. Zimmermann (2012). Microprobe sampling-photo ionization-time-of-flight mass spectrometry for in situ chemical analysis of pyrolysis and combustion gases: Examination of the thermo-chemical processes within a burning cigarette. Analytica Chimica Acta 714: 104-113.

Higgins, C. E., W. H. Griest and M. R. Guerin (1984). Sampling and analysis of cigarette smoke using the solid adsorbent tenax. Technical Report. United States, Oak Ridge National Lab., TN (USA): 23p.

Hosono, K., A. Kanazawa, H. Mori and T. Endo (2007). Photodegradation of cellulose acetate film in the presence of benzophenone as a photosensitizer. Journal of Applied Polymer Science 105(6): 32353239.

Hu, N., W. Du and Y. Dai (2015). Determination of major phenolic compounds retained by cigarette filter with high performance liquid chromatography. Tobacco Science and Technology 48(4): 49-55.

Huang, G., Y. Fang, H. Yu, L. Tao and X. Tang (2013). The determination of lead (pb) in cigarette filter using graphite furnace atomic absorption spectrometry with microwave assisted digestion. Acta Tabacaria Sinica 19(4): 5-10.

Huang, H., H. Liang, X. Liu and Y. Yu (2014). Determination of vocs in cigarette tipping paper by hs-gc/ms. Acta Tabacaria Sinica 20(1): 15-20.

Huang, Y. B., S. X. Tuo, Y. Zhao, X. Y. Xiong, Q. Yang, Y. Y. Chen, B. Chen and H. W. Yang (2014). Gc-ms investigation of the transfer behavior of alkalescent flavors in moderate/low-tar cigarettes. Chromatographia 77(1-2): 171-178.

Ishizu, Y. and Y. Ishizu (2013). A new diffusive sampler for determining nicotine and 3-ethenylpyridine contents in second-hand smoke. Indoor and Built Environment 22(3): 520-527.

ISO3308 (2012). Routine analytical cigarette-smoking machine - definitions and standard conditions. International Standard.

Jenkins, R. A., R. B. Quincy and M. R. Guerin (1979a). Selected constituents in the smokes of u.S. Commercial cigaretts: Tar, nicotine, carbon monoxide and carbon dioxide. United States: 46p.

Jenkins, R. A., R. B. Quincy and M. R. Guerin (1979b). Selected constituents in the smokes of foreign commercial cigaretts: Tar, nicotine, carbon monoxide, and carbon dioxide. United States: 62p.

Ji, H., J. Man, J. Liu, N. Liu, F. Wang and W. Han (2015). Determination of benzene compounds in mainstream cigarette smoke entrapped by filter using static headspace gc/ms method. Acta Tabacaria Sinica 21(2): 23-28.

John Dane, A., K. J. Voorhees and R. B. Cody (2002). The identification of nitropolycyclic aromatic hydrocarbons in tobacco smoke using electron monochromator mass spectrometry. Proceedings - 
50th ASMS Conference on Mass Spectrometry and Allied Topics, June 2, 2002 - June 6, 2002, Orlando, FL, United states, American Society for Mass Spectrometry.

Kadir, A. A. and N. A. Sarani (2015). Cigarette butts pollution and environmental impact - a review. Applied Mechanics and Materials 773-774: 1106-1110.

Kowalski, R. and J. Wiercinski (2009). Mercury content in smoke and tobacco from selected cigarette brands. Ecological Chemistry and Engineering S-Chemia I Inzynieria Ekologiczna S 16(2): 155-162.

Li, S., J. L. Banyasz, M. E. Parrish, J. Lyons-Hart and K. H. Shafer (2002). Formaldehyde in the gas phase of mainstream cigarette smoke. Journal of Analytical and Applied Pyrolysis 65(2): 137-145.

Liang, C. K. and J. F. Pankow (1996). Gas/particle partitioning of organic compounds to environmental tobacco smoke: Partition coefficient measurements by desorption and comparison to urban particulate material. Environmental Science \& Technology 30(9): 2800-2805.

Little, J. C., C. J. Weschler, W. W. Nazaroff, Z. Liu and E. A. Cohen Hubal (2012). Rapid methods to estimate potential exposure to semivolatile organic compounds in the indoor environment. Environ Sci Technol 46(20): 11171-11178.

Liu, C., K. G. McAdam and T. A. Perfetti (2011). Some recent topics in cigarette smoke science. MiniReviews in Organic Chemistry 8(4): 349-359.

Liu, H., J. Guo, S. Sun, C. Nie, Y. Zong and J. Xie (2013). Effects of auxiliary material parameters on deliveries of free radicals in mainstream cigarette smoke. Tobacco Science and Technology(7): 6367.

Liu, X. Y., L. Ma, J. Zhou, Y. J. Ma, R. S. Bai and L. H. Yan (2014). The influence of nitrogen dioxide on the determination of hydrogen cyanide in mainstream and sidestream cigarette smoke and the improvement of the determination method by continuous flow analyzer. Analytical Methods 6(24): 9841-9849.

Lodovici, M., V. Akpan, C. Evangelisti and P. Dolara (2004). Sidestream tobacco smoke as the main predictor of exposure to polycyclic aromatic hydrocarbons. Journal of Applied Toxicology 24(4): 277-281.

Luceri, F., G. Pieraccini, G. Moneti and P. Dolara (1993). Primary aromatic-amines from side-stream cigarette-smoke are common contaminants of indoor air. Toxicology and Industrial Health 9(3): 405-413.

Marcilla, A., M. I. Beltran, A. Gomez-Siurana, I. Martinez and D. Berenguer (2015). Effect of the concentration of siliceous materials added to tobacco cigarettes on the composition of the smoke generated during smoking. Industrial \& Engineering Chemistry Research 54(6): 1916-1929.

Masoudi Soltani, S. and S. Kazemi Yazdi (2012). Effect of dry physical mixing of cigarette filters as carbon source with koh on final physical characteristics of the synthesized porous carbon. 2012 12th IEEE International Conference on Nanotechnology, NANO 2012, August 20, 2012 - August 23, 2012, Birmingham, United kingdom, IEEE Computer Society.

McGee, D., T. Brabson, J. McCarthy and M. Picciotti (1995). 4-year review of cigarette ingestions in children. Pediatric Emergency Care 11(1): 13-16.

Merckel, C. and F. Pragst (2007). Tobacco additives in cigarettes - intended purpose and potential of danger. Journal Fur Verbraucherschutz Und Lebensmittelsicherheit-Journal of Consumer Protection and Food Safety 2(3): 287-301. 
Minzae, L., K. Gil-Pyo, S. Hyeon Don, P. Soomin and Y. Jongheop (2014). Preparation of energy storage material derived from a used cigarette filter for a supercapacitor electrode. Nanotechnology 25(34): 345601 (345 608 pp.).

Moerman, J. W. and G. E. Potts (2011). Analysis of metals leached from smoked cigarette litter. Tobacco Control 20: 130-135.

Nagarajan, S. (2014). Harnessing energy in wasted toxic materials. 32nd West Coast Energy Management Congress, EMC 2014, June 25, 2014 - June 26, 2014, Seattle, WA, United states, AEE Energy Books.

Novotny, T. E., S. N. Hardin, L. R. Hovda, D. J. Novotny, M. K. McLean and S. Khan (2011). Tobacco and cigarette butt consumption in humans and animals. Tobacco Control 20: I17-I20.

Novotny, T. E. and F. Zhao (1999). Consumption and production waste: Another externality of tobacco use. Tobacco Control 8(1): 75-80.

O'Connor, R. J., D. Hammond, A. McNeill, B. King, L. T. Kozlowski, G. A. Giovino and K. M. Cummings (2008). How do different cigarette design features influence the standard tar yields of popular cigarette brands sold in different countries? Tobacco Control 17: I1-I5.

P.R. Nelson, F. W. C., and S.P. Kelly, R.J. Reynolds (1998). Comparison of environmental tobacco smoke to aged and diluted sidestream smoke. J. Armsol Sci 29: 281-282.

Patel, V., G. W. Thomson and N. Wilson (2013). Cigarette butt littering in city streets: A new methodology for studying and results. Tobacco Control 22(1): 59-62.

Pelit, F. O., R. E. Demirdogen and E. Henden (2013). Investigation of heavy metal content of turkish tobacco leaves, cigarette butt, ash, and smoke. Environmental Monitoring and Assessment 185(11): 9471-9479.

Petraru, C., D. Balalau, M. Ilie and C. Balalau (2013). Evaluation of different kind of cigarette filters ability of to retain the toxic compounds of the vapor phase. A comparative graphical study. Farmacia 61(4): 736-741.

Pieraccini, G., S. Furlanetto, S. Orlandini, G. Bartolucci, I. Giannini, S. Pinzauti and G. Moneti (2008). Identification and determination of mainstream and sidestream smoke components in different brands and types of cigarettes by means of solid-phase microextraction-gas chromatography-mass spectrometry. Journal of Chromatography A 1180(1-2): 138-150.

Polzin, G. M., W. J. Wu, X. Z. Yan, J. M. McCraw, S. Abdul-Salaam, A. D. Tavakoli, L. Q. Zhang, D. L. Ashley and C. H. Watson (2009). Estimating smokers' mouth-level exposure to select mainstream smoke constituents from discarded cigarette filter butts. Nicotine \& Tobacco Research 11(7): 868-874.

Puls, J., S. A. Wilson and D. Holter (2011). Degradation of cellulose acetate-based materials: A review. Journal of Polymers and the Environment 19(1): 152-165.

Purkis, S. W., V. Troude, G. Duputie and C. Tessier (2010). Limitations in the characterisation of cigarette products using different machine smoking regimes. Regulatory Toxicology and Pharmacology 58(3): 501-515.

Qin, L., J. Wen, D. Ding, W. Du, B. Peng, X. Zhang, F. Xie, H. Liu and K. Zhong (2014). Filtration and retention characteristics of crotonaldehyde in cigarette filters. Tobacco Science and Technology 47: 64-70. 
Robertson, R. M., W. C. Thomas, J. N. Suthar and D. M. Brown (2012). Accelerated degradation of cellulose acetate cigarette filters using controlled-release acid catalysis. Green Chemistry 14(8): 2266.

Sabzali, A., M. Nikaeen and B. Bina (2012). Performance evaluation of cigarette filter rods as a biofilm carrier in an anaerobic moving bed biofilm reactor. Environmental Technology 33(15): 1803-1810.

Saidi, M. S., M. R. Hajaligol, A. Mhaisekar and M. Subbiah (2007). A 3d modeling of static and forward smoldering combustion in a packed bed of materials. Applied Mathematical Modelling 31(9): 19701996.

Saidi, M. S., A. Mhaisekar, M. R. Hajaligol and M. Subbiah (2006). Effects of thermo-physical and flow parameters on the static and dynamic burning of a cigarette. Combustion Theory and Modelling 10(6): 939-960.

Salman, M. S., S. K. Yazdi, S. Hosseini and M. K. Gargari (2014). Effect of nitric acid modification on porous characteristics of mesoporous char synthesized from the pyrolysis of used cigarette filters. Journal of Environmental Chemical Engineering 2(3): 1301-1308.

Schick, S. F., K. F. Farraro, C. Perrino, M. Sleiman, G. van de Vossenberg, M. P. Trinh, S. K. Hammond, B. M. Jenkins and J. Balmes (2014). Thirdhand cigarette smoke in an experimental chamber: Evidence of surface deposition of nicotine, nitrosamines and polycyclic aromatic hydrocarbons and de novo formation of nnk. Tobacco Control 23(2): 152-159.

Schick, S. F., G. van den Vossenberg, A. Luo, A. Whitlatch, P. Jacob, J. Balmes and D. Shusterman (2013). Thirty minute-exposure to aged cigarette smoke increases nasal congestion in nonsmokers. Journal of Toxicology and Environmental Health-Part a-Current Issues 76(10): 601-613.

Seco Pon, J. P. and M. E. Becherucci (2012). Spatial and temporal variations of urban litter in mar del plata, the major coastal city of argentina. Waste Management 32(2): 343-348.

Slaughter, E., R. M. Gersberg, K. Watanabe, J. Rudolph, C. Stransky and T. E. Novotny (2011). Toxicity of cigarette butts, and their chemical components, to marine and freshwater fish. Tobacco Control 20: 125-129.

Sleiman, M., R. L. Maddalena, L. A. Gundel and H. Destaillats (2009). Rapid and sensitive gas chromatography-ion-trap tandem mass spectrometry method for the determination of tobaccospecific n-nitrosamines in secondhand smoke. J Chromatogr A 1216(45): 7899-7905.

Smith, C. J., S. D. Livingston and D. J. Doolittle (1997). An international literature survey of "iarc group i carcinogens" reported in mainstream cigarette smoke. Food and Chemical Toxicology 35(10-11): 1107-1130.

Smith, S. D. A., C. L. Gillies and H. Shortland-Jones (2014). Patterns of marine debris distribution on the beaches of rottnest island, western australia. Marine Pollution Bulletin 88(1-2): 188-193.

Soltani, S. M., S. K. Yazdi, S. Hosseini and I. Bayestie (2015). Lead removal from aqueous solution using non-modified and nitric acid-modified charred carbon from the pyrolysis of used cigarette filters. Desalination and Water Treatment 53(1): 126-138.

St Charles, F. K., A. A. Kabbani and M. F. Borgerding (2010). Estimating tar and nicotine exposure: Human smoking versus machine generated smoke yields. Regulatory Toxicology and Pharmacology 56(1): 100-110. 
Suarez-Rodriguez, M. and C. M. Garcia (2014). There is no such a thing as a free cigarette; lining nests with discarded butts brings short-term benefits, but causes toxic damage. Journal of Evolutionary Biology 27(12): 2719-2726.

Suarez-Rodriguez, M., I. Lopez-Rull and C. M. Garcia (2013). Incorporation of cigarette butts into nests reduces nest ectoparasite load in urban birds: New ingredients for an old recipe? Biology Letters 9(1).

Sun, Y., W. Li, J. Wang, J. Bi and S. Su (2012). Determination of rutin in cigarette tobacco, filters, mainstream smoke and burned ash of different branded cigarettes by high performance liquid chromatography. Molecules 17(4): 3751-3760.

Sun, Y. S., W. Li, J. H. Wang, J. J. Bi and S. D. Su (2012). Determination of rutin in cigarette tobacco, filters, mainstream smoke and burned ash of different branded cigarettes by high performance liquid chromatography. Molecules 17(4): 3751-3760.

Torjussen, W., H. Zachariasen and I. Andersen (2003). Cigarette smoking and nickel exposure. Journal of Environmental Monitoring 5(2): 198-201.

Valavanidis, A. and E. Haralambous (2001). A comparative study by electron paramagnetic resonance of free radical species in the mainstream and sidestream smoke of cigarettes with conventional acetate filters and 'bio-filters'. Redox Report 6(3): 161-171.

Verdolotti, L., A. Salerno, R. Lamanna, A. Nunziata, P. Netti and S. lannace (2012). A novel hybrid pualumina flexible foam with superior hydrophilicity and adsorption of carcinogenic compounds from tobacco smoke. Microporous and Mesoporous Materials 151: 79-87.

Wagner, K. A., R. McDaniel and D. Self (2001). Collection and preparation of sidestream cigarette smoke for trace elemental determinations by graphite furnace atomic absorption spectrometry and inductively coupled plasma mass spectrometry. Journal of Aoac International 84(6): 1934-1940.

Wang, W. and B. J. Finlayson-Pitts (2003). Measurement of trace metals in tobacco and cigarette ash by inductively coupled plasma-atomic emission spectroscopy. Journal of Chemical Education 80(1): 8385.

Wang, Y., X.-Y. Yao, D. Li, X. Jin, Q.-C. Li, B. Sun, Y.-H. Han, L. Fan and Q.-P. Qi (2007). Determination of hg and as in cigarette filter components with hydride generation-atomic fluorescence spectrometry. Tobacco Science and Technology(9): 41-45.

Waters, H. (2013). Bird butts used cigarette filters in nests may protect hatchlings. Scientific American 308(2): 24-24.

Watson, C., J. McCraw, G. Polzin and D. Ashley (2004). Development of a method to assess cigarette smoke intake. Environmental Science \& Technology 38(1): 248-253.

Watson, C. H., J. S. Trommel and D. L. Ashley (2004). Solid-phase microextraction-based approach to determine free-base nicotine in trapped mainstream cigarette smoke total particulate matter. Journal of Agricultural and Food Chemistry 52(24): 7240-7245.

Wilson, N., J. Oliver and G. Thomson (2014). Smoking close to others and butt littering at stops: Pilot observational study. Peerj 2.

Wright, S. L., D. Rowe, M. J. Reid, K. V. Thomas and T. S. Galloway (2015). Bioaccumulation and biological effects of cigarette litter in marine worms. Scientific Reports $\mathbf{5}$. 
Wu, D., S. Landsberger and S. M. Larson (1997). Determination of the elemental distribution in cigarette components and smoke by instrumental neutron activation analysis. Journal of Radioanalytical and Nuclear Chemistry 217(1): 77-82.

Wu, Y. P., Q. F. Zhang, L. Wang, Z. B. Su, B. He and G. Y. Yang (2012). Determination of trace lead in cigarette paper by flame atomic absorption spectrometry. Asian Journal of Chemistry 24(1): 126128.

Xia, G., L. Ma, H. Huang and X. Zhang (2012). Comparison of hazard indexes of some domestic and imported cigarettes. Tobacco Science and Technology(6): 37-40.

Xie, Y. Q., H. W. Tong, X. Y. Yan, X. P. Yang, B. Ding and S. M. Liu (2012). Determination of 14 polycyclic aromatic hydrocarbons in mainstream smoke from flue-cured cigarettes by gc-ms using a new internal standard. Asian Journal of Chemistry 24(8): 3499-3503.

Yang, K., H. Cui, H. Li, L. Fan, L. Zhang, X. Wu, Z. Li, A. Wang, Y. Liu and M. Ji (2015). On-line analysis of puff-by-puff release characteristics of co in gas phase of mainstream cigarette smoke by tdlas technology. Tobacco Science and Technology 48(10): 36-42.

You, J., G. Zhu, Y. Zhang and J. Ni (2014). Determination of menthol in mentholated cigarettes by headspace gas chromatography. Tobacco Science \&amp; Technology(8): 51-54.

Yu, J., S. Wang, B. Wang, X. Zhao, J. Cai, Q. Yan, L. Pan, F. Xie and X. Zhang (2013). Determination of eight volatile carbonyl compounds in cigarette filter by lc-esi-ms/ms. Tobacco Science and Technology(9): 39-46.

Zhang, H., N. Liu, Z. Bian, G. Tang and Q. Hu (2012). Determination of phthalic acid esters in cigarette filter by gc/ms. Tobacco Science \&amp; Technology(3): 52-56.

Zhang, H. F., G. L. Tang, N. Liu, Z. Y. Bian and Q. Y. Hu (2012). The fate of maleic hydrazide on tobacco during smoking. Scientific World Journal.

Zhang, X. T., H. W. Hou, L. K. Shi, Y. Liu, A. Wang and Q. Y. Hu (2014). Novel method to analysis benzo a pyrene in filter by liquid chromatography/tandem mass spectrometry: Application to assess mouth level benzo a pyrene exposure. Rapid Communications in Mass Spectrometry 28(13): 1468-1472.

Zhao, J. and P. K. Hopke (2012). Concentration of reactive oxygen species (ros) in mainstream and sidestream cigarette smoke. Aerosol Science and Technology 46(2): 191-197.

Zhao, J., N. S. Zhang, C. T. Qu, X. M. Wu, J. T. Zhang and X. Zhang (2010b). Cigarette butts and their application in corrosion inhibition for $\mathrm{n} 80$ steel at 90 degrees $\mathrm{c}$ in a hydrochloric acid solution. Industrial \& Engineering Chemistry Research 49(8): 3986-3991.

Zhao, J., N. S. Zhang, C. T. Qu, J. T. Zhang and X. A. Zhang (2010a). Comparison of the corrosion inhibitive effect of anaerobic and aerobic cigarette butts water extracts on n80 steel at 90 degrees $\mathrm{c}$ in hydrochloric acid solution. Industrial \& Engineering Chemistry Research 49(24): 12452-12460.

Zheng, S., B. Liu, Z. Xu, W. Sun and D. Wu (2014). Analysis of gas phase components in mainstream cigarette smoke by bag sampling-thermal desorption-gc/ms. Acta Tabacaria Sinica 20(2): 9-17. 